\title{
Neural and cognitive determinants of smoking addiction and cessation
}

Citation for published version (APA):

Havermans, A. (2016). Neural and cognitive determinants of smoking addiction and cessation. [Doctoral Thesis, Maastricht University]. https://doi.org/10.26481/dis.20160513ah

Document status and date:

Published: 01/01/2016

DOI:

10.26481/dis.20160513ah

Document Version:

Publisher's PDF, also known as Version of record

\section{Please check the document version of this publication:}

- A submitted manuscript is the version of the article upon submission and before peer-review. There can be important differences between the submitted version and the official published version of record.

People interested in the research are advised to contact the author for the final version of the publication, or visit the DOI to the publisher's website.

- The final author version and the galley proof are versions of the publication after peer review.

- The final published version features the final layout of the paper including the volume, issue and page numbers.

Link to publication

\footnotetext{
General rights rights.

- You may freely distribute the URL identifying the publication in the public portal. please follow below link for the End User Agreement:

www.umlib.nl/taverne-license

Take down policy

If you believe that this document breaches copyright please contact us at:

repository@maastrichtuniversity.nl

providing details and we will investigate your claim.
}

Copyright and moral rights for the publications made accessible in the public portal are retained by the authors and/or other copyright owners and it is a condition of accessing publications that users recognise and abide by the legal requirements associated with these

- Users may download and print one copy of any publication from the public portal for the purpose of private study or research.

- You may not further distribute the material or use it for any profit-making activity or commercial gain

If the publication is distributed under the terms of Article $25 \mathrm{fa}$ of the Dutch Copyright Act, indicated by the "Taverne" license above, 


\section{NEURAL AND COGNITIVE DETERMINANTS OF SMOKING ADDICTION AND CESSATION}


(C) Anne Havermans, 2016

Cover photograph: Thomas Herbrich

Cover design: Anne Havermans \& Job van den Hurk

Production: CPI Wöhrman Print Service B.V.

ISBN: 978-94-6328-037-2

The work presented in this thesis was funded by a TOP grant from The Netherlands Organization for Health Research and Development (ZonMW). 


\title{
NEURAL AND COGNITIVE DETERMINANTS OF SMOKING ADDICTION AND CESSATION
}

\author{
DISSERTATION \\ To obtain the degree of doctor at Maastricht University, \\ on the authority of Rector Magnificus, Prof Dr. L.L.G. Soete, \\ in accordance with the decision of the Board on Deans, \\ to be defended in public on Friday May 13 th 2016 at 12.00 hours \\ by
} Anne Havermans 


\section{Promotores}

Prof. dr. W.J. Riedel

Prof. dr. C.P. van Schayck

\section{Copromotor}

Dr. E.F.P.M. Vuurman

\section{Beoordelingscommissie}

Prof. dr. J.G. Ramaekers (voorzitter)

Prof. dr. D. Kotz (Heinrich-Heine University Düsseldorf)

Dr. S. Mulkens

Prof. dr. M.R. Munafo (University of Bristol) 


\section{TABLE OF CONTENTS}

Introduction

Chapter 1

Treatment with a nicotine vaccine does not lead to changes in brain activity during smoking cue exposure or a working memory task.

\section{Chapter 2}

Nicotine deprivation elevates neural representation of smoking-related cues in object-sensitive visual cortex.

\section{Chapter 3}

Neural representation of smoking-related cues under influence of smoking deprivation: a multivariate searchlight approach.

\section{Chapter 4}

How to win -and not only fight- a running battle: Web-based attention bias modification training for active smokers.

\section{Chapter 5}

Predictors of successful attention bias modification for smokers.

General discussion

Summary

Samenvatting

Supplementary data

Valorisation addendum

Dankwoord 



\section{INTRODUCTION}

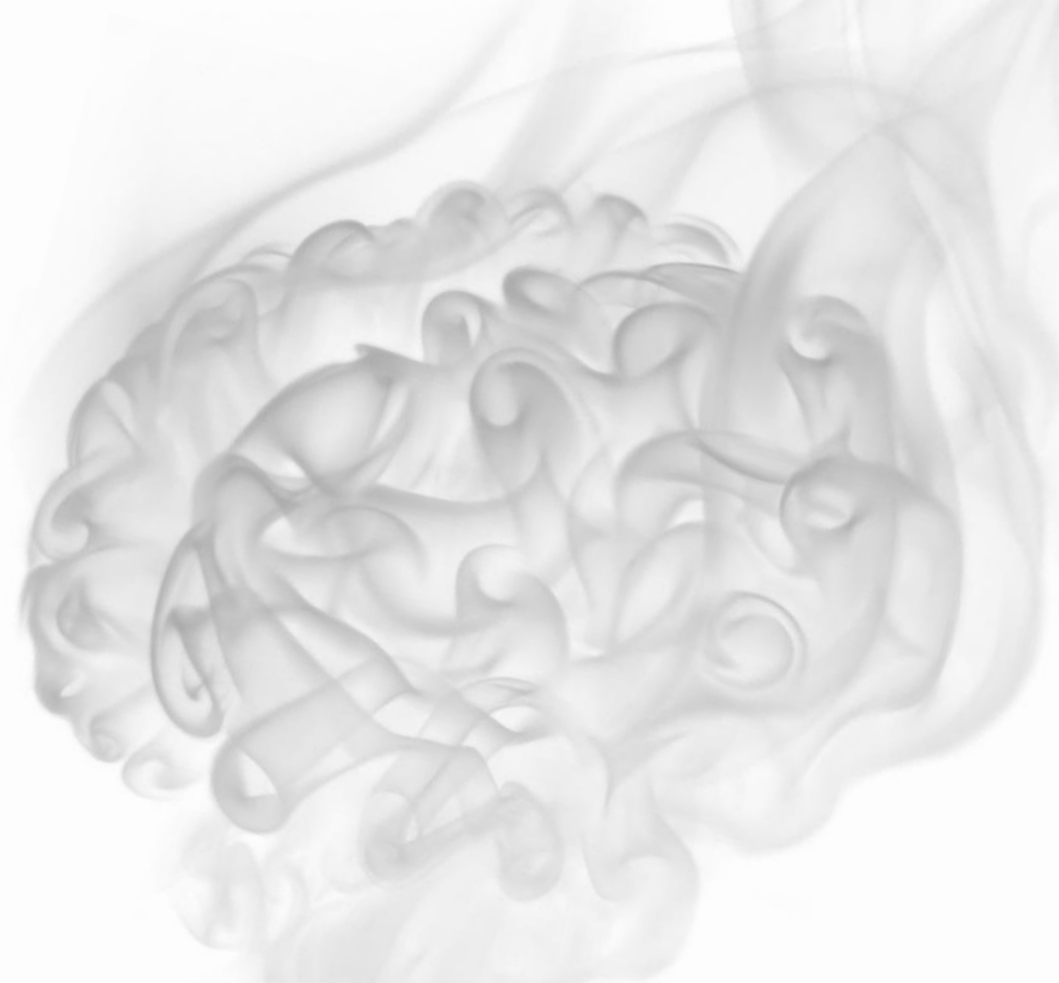


The deserted plains of Western America: a vast and powerful prairie landscape, occasionally disturbed by some passing herds of cattle or wild horses. The sun slowly sinks into the distant horizon. A galloping stallion approaches from the distance, carrying a man wearing leather boots and a cowboy hat. As he casts a sultry glance at you, the man lights up a cigarette and inhales deeply. This may sound like the start of a superficial romantic novel, but in fact this setting was created by a tobacco company with the purpose of persuading people (particularly men) to buy their products during the 1950's, -60's and 70 's. In this iconic and highly successful campaign, smoking cigarettes was associated with being sturdy and masculine, like the cowboys - also known as Marlboro men. But, times have changed and the once so gloriously portrayed Marlboro men have all died inglorious deaths as a result of their once admired smoking habit. Fortunately our knowledge of tobacco's health-damaging and addictive properties has increased extensively over the years and exalting advertising has been banned accordingly. Nevertheless, more than one billion people worldwide still smoke tobacco. Moreover, approximately six million people die each year as a result of direct tobacco use or exposure to second-hand smoke, and this number keeps increasing (World Health Organization, 2015). Many smokers are aware of the detrimental consequences of smoking, and most of those who are - want to quit. However, despite all the current knowledge, smoking cessation has not become much easier since the time of the old cowboys. Various pharmacological and behavioral therapies have become available to aid in smoking cessation. However, even with the most successful combination of both, one year successful abstinence rates remain well below $50 \%$ (Fiore et al., 2008; Rigotti, 2013). Better treatments are clearly necessary, but in order to get there it is important to understand why smoking is so tremendously addicting.

\section{Neuropharmacological effects of nicotine}

The main causal factor in smoking addiction is nicotine. Nicotine is regarded one of the most harmful and addictive commonly used substances (Nutt, King, Saulsbury, \& Blakemore, 2007). An important reason for nicotine's extremely high addiction potential is its route of administration. After inhalation from a cigarette, nicotine readily passes via the lungs into the blood stream and reaches the brain within 7 seconds (Jiloha, 2010; Rosecrans $\&$ Karan, 1993). This rapid rise of nicotine levels causes immediate reinforcement, and the close proximity between action and outcome facilitates strong conditioning of this behavior. Drugs that are administered through other routes, for instance intravenously, take much longer than nicotine to assert their effects on the brain. Also administration of nicotine through different routes, such as in gum or patches, causes a much more gradual 
increase of blood nicotine levels, and thus a slower supply to the brain (Benowitz, 2008a; Hukkanen, Jacob, \& Benowitz, 2005). Therefore, these types of nicotine replacement therapies (NRT) are less addictive than cigarettes and can be effectively applied for smoking cessation.

Nicotine binds to nicotinic acetylcholine receptors (nAChR's) which are located at different sites in the peripheral and central nervous system (Feduccia, Chatterjee, \& Bartlett, 2012). Numerous nACh receptor subtypes have been identified. When nicotine activates these receptors, various neurotransmitters are released, depending on the receptor subtype and location. These in turn mediate different cognitive and behavioral functions. In particular, nicotine has a high affinity for receptors of the $\alpha 4 \beta 2$ subtype, which, when activated, promote the release of dopamine in the frontal cortex and mesolimbic areas (Benowitz, 2008b, 2009). The release of dopamine in this 'reward pathway', particularly in the shell of the nucleus accumbens, signals a pleasurable experience, which underlies nicotine's positive reinforcing effects. Besides its role in pleasure and reward, nicotine can also influence arousal and various cognitive functions such as attention and working memory.

Longer periods of nicotine exposure induce chronic changes in the brain, which set off the transition to addiction. When acetylcholine is released in the synaptic cleft, some of it binds to the nAChR's while the rest of it is broken down by certain enzymes. However, these enzymes do not break down left-over nicotine. Therefore, after repeated inhalation, low levels of nicotine continue to surround the nAChR's, causing them to gradually become unresponsive. As a consequence, administration of the same amount of nicotine has diminishing effects with continued use, i.e. acute dependence. This desensitization happens only to some specific cholinergic receptors, causing glutamatergic excitation of dopaminergic neurons to persist, while GABAergic inhibition diminishes (Benowitz, 2010). As a result the rewarding dopamine response to nicotine becomes much stronger. Moreover, in reaction to this desensitization, an upregulation of receptors and binding sites takes place. When after a (short) period of abstinence, all the desensitized nAChR's become responsive again, this leads to heightened sensitivity to nicotine (Benowitz, 2010). Further, withdrawal symptoms arise after re-sensitization of nAChR's (Benowitz, 2008b). In sum, long-term nicotine exposure leads to neural changes that enhance the reward system's responsiveness to nicotine and thereby cause a smoker to become extremely sensitive to (nicotinic) rewards. 


\section{Unbalanced neural networks in smoking addiction}

The strong dopaminergic reward caused by nicotine is not sufficient to cause addiction, as it is also seen in non-addicted substance users. Moreover, reasons for smoking reported by long-term smokers typically do not only include this 'pleasurable experience', but are often related to habits, social situations, or alleviation of craving and/or withdrawal symptoms (Piasecki, Richardson, \& Smith, 2007; Shiffman, Dunbar, Scholl, \& Tindle, 2012). Indeed, multiple neural systems have been implicated in addiction. Essentially, it has been suggested that there is an imbalance between an ineffective cognitive control system (topdown) based in the prefrontal cortex (PFC) and an overactive motivational system (bottomup) involving the amygdala and ventral striatum (Bechara, 2005; Goldstein \& Volkow, 2011; Koob \& Volkow, 2010; Noël, Brevers, \& Bechara, 2013; Volkow, Fowler, \& Wang, 2004; Volkow, Wang, Tomasi, \& Baler, 2013; Volkow, 2002). Cognitive control circuits in the brain are involved in the guidance of goal directed behavior, for instance by making decisions based on short- and long-term outcomes, and by inhibiting responses to distracting stimuli (Watanabe, Hikosaka, Sakagami, \& Shirakawa, 2002). Substance dependent individuals are typically characterized by poor decision making (i.e. choosing to continue using a drug, in spite of negative consequences) and an inability to control their behavior in response to substance use. In line with this, reduced neural activity in prefrontal cortical regions has been demonstrated in smokers during inhibitory cognitive control (de Ruiter, Oosterlaan, Veltman, van den Brink, \& Goudriaan, 2012; Luijten et al., 2013; Nestor, McCabe, Jones, Clancy, \& Garavan, 2011). Moreover, ex-smokers showed increased activity in prefrontal cortical regions compared to both non-smoking controls and smokers (Nestor et al., 2011), indicating that elevated top-down control may be a significant feature of successful abstinence.

Besides reduced neural activation in top-down cognitive processing, smokers exhibit augmented bottom-up neural activity in structures which trigger affective responses to pleasant or aversive salient stimuli. Accordingly, nicotine acquires powerful affective and emotional properties in smokers by eliciting dopamine in a more prolonged and unregulated manner than natural rewarding stimuli do (Koob \& Volkow, 2010). This dopamine release also facilitates the associative learning of an incentive value of environmental cues that are paired with the reinforcing outcome of smoking (Chiamulera, 2005; Martin-Soelch, 2013; Robinson \& Berridge, 2008). On their own, these cues do not evoke pleasurable sensations, but they signal the possibility of future reward and induce a state of 'wanting' by activating the mesolimbic system (Hester \& Luijten, 2013). Over time drug-related cues can become extremely salient and attention grabbing and provoke craving and smoking behavior (Benowitz, 2010; Robinson \& Berridge, 2008). Neuroimaging 
studies in smokers have demonstrated increased activity in a common range of areas (e.g. amygdala, ventral striatum, thalamus and cingulate and orbitofrontal cortex) in response to smoking-related cues (David et al., 2005; Due, Huettel, Hall, \& Rubin, 2002; Franklin et al., 2007; Janes, Pizzagalli, Richardt, Frederick, Holmes, et al., 2010; McBride, Barrett, Kelly, Aw, \& Dagher, 2006; Rubinstein et al., 2010; Smolka et al., 2006; Wilson, Sayette, $\&$ Fiez, 2004). Thus, exaggerated incentive salience of smoking cues and accompanying responses in the brain's motivational meso-cortico-limbic circuit are likely to play an important role in tobacco seeking behavior. In addition, positive reinforcing effects of drugs are thought to decrease with prolonged use, whereas negative reinforcing mechanisms gain a stronger influence in the motivation to use a substance (Bechara, 2005; Koob, 2009, 2013). The most important negative reinforcing factor for tobacco smoking is the relief of withdrawal symptoms such as stress, negative mood and dysphoria. Such withdrawal signs invigorate the incentive impact of nicotine, thereby increasing the motivation to smoke. When the bottom-up incentive signals triggered in the motivational system become very strong, they may overpower top-down cognitive control, causing smokers to not be able to resist lighting up another cigarette (Bechara, 2005). In this way, finally a repetitive and automatic pattern of behavior arises that is directed excessively and compulsively towards smoking behavior.

\section{Attention bias in smokers}

An essential consequence of the over-sensitized motivational system in smokers is the development of an attention bias for substance related cues. Attention bias is the fast and automatic process of focusing and engaging attention on salient smoking related cues in the smokers' environments (Field \& Cox, 2008; Franken, 2003; Hogarth, Mogg, Bradley, Duka, \& Dickinson, 2003; Mogg \& Bradley, 2002). As described earlier, these cues have gained exaggerated motivational value and often trigger craving, leading to smoking behavior. Consequently, attention bias is thought to play an important role in the maintenance of smoking dependence.

Functional imaging studies have shown hyper-activation associated with attention bias in smokers in neural regions involved in identification of -and responses to- emotionally salient stimuli (i.e. nucleus accumbens (Nestor et al., 2011), amygdala (Janes, Pizzagalli, Richardt, Frederick, Holmes, et al., 2010; Nestor et al., 2011) and insula (Janes, Pizzagalli, Richardt, Frederick, Holmes, et al., 2010; Luijten et al., 2011), as well as in areas that constitute a dorsal top-down attentional system (i.e. the dorsal Anterior Cingulate Cortex, 
right Superior parietal lobule, and the left Superior Temporal Gyrus (Janes, Pizzagalli, Richardt, Frederick, Holmes, et al., 2010; Luijten et al., 2011; Nestor et al., 2011). Activation of these areas clearly reflects the process of enhanced top-down activation needed to compensate the effects of the automatic distraction by salient smoking cues. In addition, increased activation of brain regions involved in visual processing has also been demonstrated in response to smoking related cues and related to attention bias (Engelmann et al., 2012; Janes, Pizzagalli, Richardt, Frederick, Holmes, et al., 2010). In line with this, it has been suggested that the amygdala can enhance visual processing of (emotionally) salient stimuli. That is, it may modulate activity either through direct feedback projections to various structures in the ventral visual processing stream, or through projections to frontal areas that control the allocation of attentional resources (Pessoa \& Ungerleider, 2004). However, up until the start of this thesis project no studies had investigated the exact underlying mechanisms of visual processing of smoking cues under influence of attention bias.

The clinical relevance of attention bias however, has become clear over the past years. Theoretical accounts have suggested that attention bias has a reciprocal relation with craving, by which each can strengthen the other, and both can lead to smoking behavior (Field \& Cox, 2008; Field, Marhe, \& Franken, 2014; Franken, 2003). That is, automatic selective processing of salient cues will increase the likelihood of smoking cues to be detected, and perception of these cues has been associated with the expectance of a drugreward, which triggers conditioned responses such as craving and ultimately smoking (Conklin et al., 2015). Craving in its turn will further enhance attention bias, as smoking related cues become more salient when smokers experience craving (Robinson \& Berridge, 2008). Some experimental studies have also demonstrated this relationship between craving and attention bias (eg. Mogg \& Bradley, 2002; Mogg, Field, \& Bradley, 2005), while others did not (eg. Hogarth et al., 2003). In line with this, a large meta-analytic study has demonstrated a modest positive correlation between craving and attention bias for tobacco related cues (Field, Munafò, \& Franken, 2009). Furthermore, attention bias to smoking cues has been associated with higher chances of relapse in smokers attempting to remain abstinent, in studies using the addiction stroop task (Janes, Pizzagalli, Richardt, Frederick, Chuzi, et al., 2010; Powell, Dawkins, West, Powell, \& Pickering, 2010; Spiegelhalder et al., 2011; Waters, Shiffman, Sayette, et al., 2003). Correspondingly, avoidance of smoking cues was related to successful abstinence (Peuker \& Bizarro, 2013). However, two experiments using the dot-probe task to assess attention bias, did not find this association (Spiegelhalder et al., 2011; Waters, Shiffman, Bradley, \& Mogg, 2003). In the end, the 
most direct approach to investigate the causal influence of attentional bias is to manipulate it and assess the effects on clinical outcomes.

\section{Attention bias modification for smokers}

Currently, six studies have investigated the effects of attention bias modification (ABM) in smokers (Attwood, Penton-Voak, \& Munafò, 2009; Begh et al., 2015; Field, Duka, Tyler, \& Schoenmakers, 2009; Kerst \& Waters, 2014; Lopes, Pires, \& Bizarro, 2014; McHugh, Murray, Hearon, Calkins, \& Otto, 2010). All of these studies have employed modified versions of the dot-probe task, in which participants are instructed to react as quickly as possible to a visual probe replacing either a smoking related or a neutral cue. Faster reactions to smoking related cues in this task indicate an attention bias towards these cues. Attention bias can be modified by manipulating the amount of trials in which the probe replaces each type of cues. So when the probe replaces neutral cues in the majority of trials, participants are implicitly trained to relocate their attention away from smoking related cues. Not many studies have investigated the effects of ABM in smokers, but the results are cautiously promising (see table 1). The first three studies employed a single session ABM approach. Attwood and colleagues showed an increased attention bias in smokers who were trained to attend smoking cues as well as a decreased attention bias in smokers who were trained to avoid smoking cues (Attwood et al., 2009). Moreover they reported increased craving in male smokers in the attend group. A study by Field et al. confirmed the results on increasing attention bias, however this seemed to be a task specific training effect as the effect was not present when different stimuli were used during assessment and training (Field, Munafò, et al., 2009). Both Field et al. and McHugh et al. did not find any effects of ABM aimed at avoiding smoking cues on attention bias or craving (Field, Duka, et al., 2009; McHugh et al., 2010). Concluding from these studies, it is likely that one single session of $\mathrm{ABM}$ is not sufficient to reduce attention bias to smoking cues or influence craving. Currently, three studies have administered multiple sessions of ABM to smokers. Lopes and colleagues found a robust decrease in attention bias, which remained present up to 6 months after the three ABM trainings aimed at avoiding smoking cues (Lopes et al., 2014). Moreover, this effect was present when attention bias was assessed with novel pictorial cues that had not been used for training, suggesting that this was not merely a taskspecific training effect but a more general desensitization for smoking related cues. The next study, by Kerst and Waters, also showed reduced attention bias, as well as diminished cue-elicited craving, after multiple sessions of avoidance ABM training (Kerst \& Waters, 2014). Finally, an ABM study by Begh and colleagues failed to find any effects on attention 
bias or clinical outcomes (Begh et al., 2015). However, the results in this study may be biased by the fact that also no baseline attention bias was demonstrated. All together, these findings indicate that multiple sessions of $\mathrm{ABM}$ may be beneficial for smokers in decreasing selective attentional processing of smoking related cues and thereby reducing craving in response to such cues. 


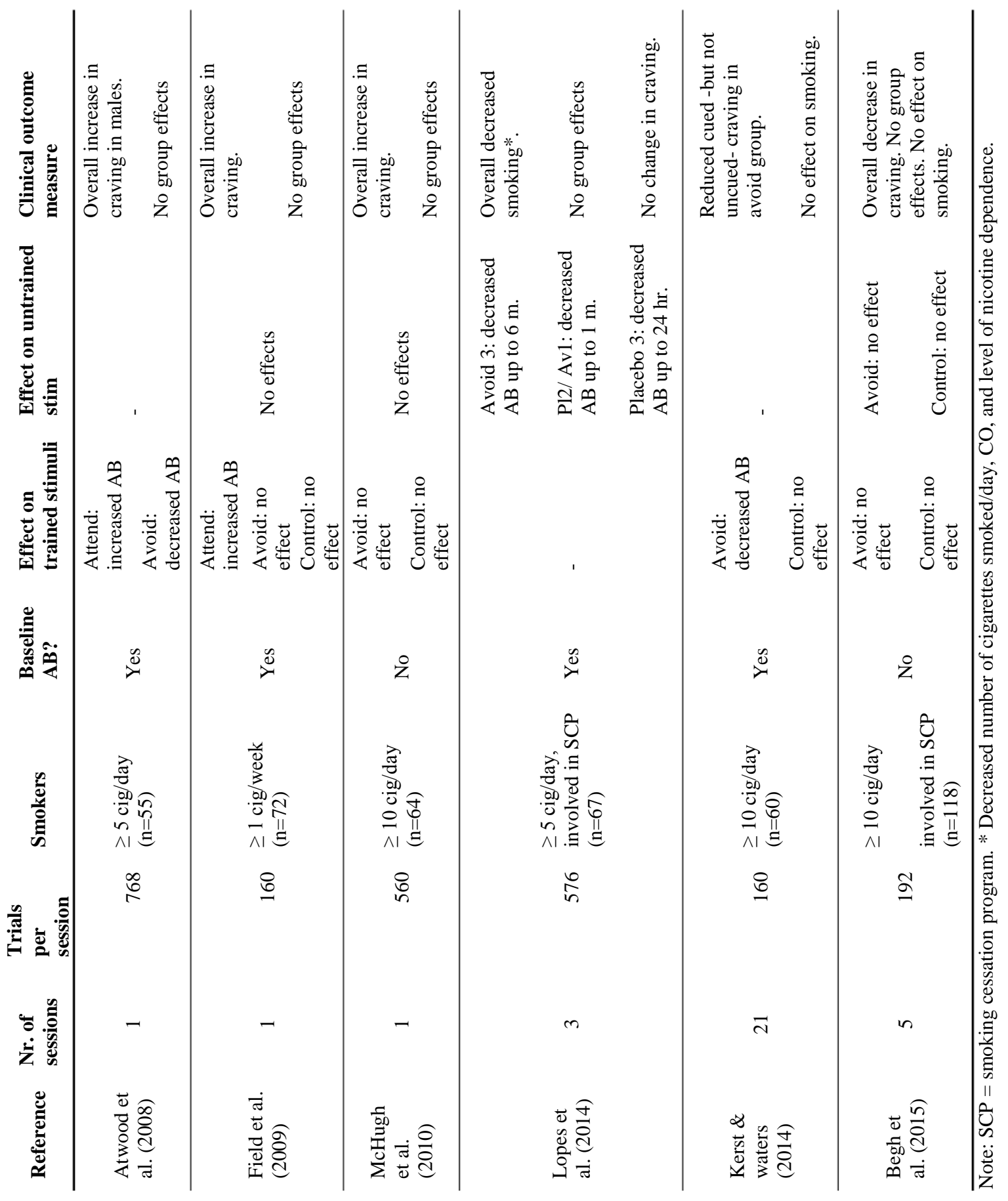




\section{Content of this thesis}

The main objective of this thesis project was to gain more knowledge about some of the neurocognitive mechanisms underlying smoking dependence, and relate this to specific smoking cessation methods. Since the success rates for quitting with current treatments remain rather low, it is of crucial importance to investigate new options. More knowledge about the neurocognitive mechanisms in smoking addiction could benefit the development of such treatments. This thesis describes our investigations based on the following research questions.

Does treatment with a nicotine vaccine lead to differences in neural and/or behavioral responses after an acute nicotine dose?

Addiction to smoking is for a large part a consequence of the actions of nicotine in the brain. Therefore one strategy for smoking cessation is to prohibit these actions by precluding nicotine from entering the brain. This is the concept of several nicotine vaccines that are currently in development. An advantage of vaccination against nicotine is that nicotine is targeted in the bloodstream, so there will be no side effects as a consequence of pharmacological interaction with receptors. However, nicotine's effects on brain and behavior are widespread, so cutting off nicotine completely may still have consequences. In our first study we explored the effects of nicotine immunotherapy on nicotine-mediated processes of cue reactivity and working memory on both the neural and behavioral level. Chapter 1 describes this randomized clinical trial in which smokers were treated with a vaccine which was expected to prevent nicotine from entering the brain. The aim of this study was to prove the concept of this vaccine, by assessing whether there were nicotinemediated differences in brain activity between vaccinated and placebo-vaccinated smokers. We hypothesized that vaccinated smokers would show no effect of an acute dose of nicotine on brain responses and performance during a working memory task, as opposed to placebo-vaccinated smokers. Moreover, lower amounts of nicotine entering the brain would decrease the reinforcing effect of smoking and consequently weaken the conditioned responses to smoking related cues. Therefore we hypothesized that vaccinated smokers would show decreased neural responses to smoking related cues. 
Can attention bias for smoking cues be traced back to a basic level of visual object processing?

Besides the direct reinforcing effects of nicotine, conditioned responses to smoking related cues also contribute to the maintenance of smoking addiction. In order to develop a smoking cessation intervention aimed at these responses, it is important to know more about their underlying mechanism. Therefore, the aim of the study described in chapter 2 was to further investigate the neural substrates of attention bias in smokers. Since emotionally salient stimuli receive enhanced sensory processing in the visual cortex, we expected this to also apply to smoking related cues- which are salient to smokers. We used fMRI and Multi-voxel pattern analysis (MVPA) to elucidate subtle differences in neural responses to smoking related and neutral cues in object-sensitive lateral occipital complex (LOC). Since smoking cues become particularly salient when smokers are deprived from nicotine, we hypothesized that neural response patterns to smoking related and neutral cues would be discriminable when smokers were nicotine deprived but not when they were satiated.

Do other brain regions show differential processing of smoking related and neutral cues under influence of attention bias?

Excessive selective processing of smoking cues is not likely to be limited to LOC only. Various motivational, attentional and visual processing areas have been implicated as well. Therefore, in the study described in chapter 3, we aimed to identify these interacting neural networks involved in attention bias for smoking cues. To achieve this, we performed a multivariate searchlight approach to map clusters of voxels that show discriminable responses to smoking related and neutral images during the nicotine deprivation, but not during satiation.

Do five sessions of web-based ABM training reduce attention bias and subjective craving in smokers?

Reducing biased attention to smoking cues could contribute to smoking cessation and the prevention of relapses. Chapter 4 describes a large web-based ABM training that aimed to decrease smokers' attention bias. Based on findings of previous $A B M$ studies, we developed a training consisting of five (semi-) daily sessions of the modified dot-probe task 
and a baseline and post-test measurement. We also aimed to assess the robustness of the ABM effect, by adding a follow-up measurement one week after the last training. In addition we used novel images during the assessment stage of the study which had not been used in the training, in order to ascertain the generalizability of the effect. We hypothesized that five sessions ABM training would diminish attention bias and possibly craving in active smokers and that this effect would still be present at follow-up. Moreover we expected that this effect would not just be present for the trained smoking cues, but would also be generalizable to novel ones.

Is the effectiveness of ABM in reducing attention bias influenced by smokers' personal characteristics?

As the results of current AMB trials for smokers are mixed, it is interesting to see whether there are specific factors influencing this effect. It is possible that ABM is effective only for a certain subset of smokers. Therefore, in the study described in chapter 5, we applied a factorial univariate analysis of variance in order to carefully examine the relationship between several characteristics of our smoking participants and their attention bias at baseline, as well as the change in attention bias after ABM or control training. 


\section{Neuroimaging methods}

Three of the studies in this thesis made use of neuroimaging analysis techniques. In chapter 2 we used fMRI and univariate general linear model analyses to find brain responses to smoking cues and a working memory task in different conditions under influence of nicotine or an anti-nicotine vaccine. Moreover, to answer the highly specific research questions in chapter 3 and 4, some advanced fMRI analysis approaches were applied. In chapter 3 we expected very subtle differences in processing of smoking related and neutral cues in a piece of occipital cortex. In order to elucidate these subtle differences, we applied multi-voxel pattern analysis (MVPA). In chapter 4 we aimed to find out whether other brain areas would also show these subtle differences in neural response patterns to smoking related and neutral cues. To achieve this we employed a multivariate searchlight approach, which allowed us to apply MVPA in the whole brain instead of in one specific region of interest. Here, I will provide a short introduction to all of these neuroimaging methods.

\section{Functional Magnetic Resonance Imaging}

Magnetic resonance imaging (MRI) is a neuroimaging method that relies on the magnetic properties of different types of tissue. In the strong static magnetic field of the MRI scanner, hydrogen protons in a person's body tend to align. The scanner emits a radiofrequency pulse, which shortly disturbs this alignment. When the pulse is switched off, the protons return to their original state and thereby emit a faint MR signal that can be detected by the scanner. In functional MRI, the contrast between oxygenated and deoxygenated hemoglobin in the blood is typically of interest. Depending on whether it is bound to oxygen or not hemoglobin has different magnetic properties, which affect the MR signal. As a result, the MR signal is slightly stronger when the blood is oxygenated. When a group of neurons is activated it will consume oxygen, and in response to this demand the supply of oxygenated blood to this area will increase rapidly. Consequently, the differences in blood oxygenation throughout the brain are measured and the resulting signal is called the Blood Oxygenation Level Dependent (BOLD) signal. In conclusion, this signal does not directly reflect neural activity, but provides a reliable measure of changes in brain activation. An important benefit of fMRI is its high spatial resolution; images can be acquired with precision up to one millimeter, depending on the magnetic field strength and particular imaging parameters. However, since fMRI does not directly measure neural activity, but the consequential changes in blood flow, there is always a slight delay before the BOLD signal can be detected, which limits its temporal resolution. 


\section{General Linear Model (GLM)}

An fMRI dataset consists of a large set of cubical elements called voxels, each of which has an associated time course that reflects the BOLD signal change in that region during the time of the experiment. The general linear model is an analysis method based on multiple regression, that aims to explain the variation of this time course in each voxel, in terms of a linear combination of several known factors and an error term. To do this, a design matrix is constructed that contains a predictor for each experimental condition, based on its onand offset times. This design matrix is used to generate a prediction of the hemodynamic response of each voxel at every time point. Then, the GLM fitting procedure estimates if, and to what extent, each predictor contributes to the variability observed in the voxel's actual time-course. Subsequently, for each predictor a beta value is obtained that quantifies this estimation for each voxel time course. Statistical maps based on these beta values can be used to visualize and compare each voxel's response to the different conditions.

\section{Multi-Voxel Pattern Analysis (MVPA)}

Whereas the GLM model assesses the time course of each voxel separately, MVPA approaches take into account the spatial distribution of neural responses. Thereby MVPA is able to detect subtle differences in neural activity patterns to different experimental conditions in a region, even when its average response to both is the same. For each trial in the experiment, response patterns are created by estimating the response from each voxel within the region of interest, and labeled according to their corresponding condition. Subsequently a classifying algorithm is trained to 'learn' the association between the response patterns and condition labels. Finally, the trained model is tested by predicting the conditions of a remaining independent subset of the data. This procedure is usually repeated numerous times with different subsets to train and test the model (cross validations). When the (average) prediction accuracy of the model lies significantly above chance level, this reflects a meaningful difference in spatial patterns of neural activity elicited by the two conditions.

\section{Multivariate Searchlight Mapping}

A specific MVPA approach that detects discriminative pattern information throughout the whole brain is multivariate searchlight mapping. With this approach an analysis (typically MVPA) is performed for each voxel, incorporating a small spherical subset of surrounding voxels. The result of this analysis (in this case a prediction accuracy) is then stored in the location of the central voxel. This way a whole brain map can be obtained that shows which clusters of voxels discriminate between the experimental conditions. 


\section{References}

Attwood, A. S., Penton-Voak, I. S., \& Munafò, M. R. (2009). Effects of acute nicotine administration on ratings of attractiveness of facial cues. Nicotine \& Tobacco Research, 11(1), 44-48.

Bechara, A. (2005). Decision making, impulse control and loss of willpower to resist drugs: a neurocognitive perspective. Nature Neuroscience, 8(11), 1458-63.

Begh, R., Munafò, M. R., Shiffman, S., Ferguson, S. G., Nichols, L., Mohammed, M. A., ... Aveyard, P. (2015). Lack of attentional retraining effects in cigarette smokers attempting cessation: A proof of concept double-blind randomised controlled trial. Drug and Alcohol Dependence, 149, $158-165$.

Benowitz, N. L. (2008a). Clinical pharmacology of nicotine: implications for understanding, preventing, and treating tobacco addiction. Clinical Pharmacology and Therapeutics, 83(4), $531-41$.

Benowitz, N. L. (2008b). Neurobiology of nicotine addiction: implications for smoking cessation treatment. The American Journal of Medicine, 121(4 Suppl 1), S3-10.

Benowitz, N. L. (2009). Pharmacology of nicotine: addiction, smoking-induced disease, and therapeutics. Annual Review of Pharmacology and Toxicology, 49, 57-71.

Benowitz, N. L. (2010). Nicotine addiction. The New England Journal of Medicine, 362, 2295-303.

Chiamulera, C. (2005). Cue reactivity in nicotine and tobacco dependence: A "multiple- action" model of nicotine as a primary reinforcement and as an enhancer of the effects of smokingassociated stimuli. Brain Research Reviews, 48, 74-97.

Conklin, C. A., Vella, E. J., Joyce, C. J., Salkeld, R. P., Perkins, K. A., \& Parzynski, C. S. (2015). Examining the relationship between cue-induced craving and actual smoking. Experimental and Clinical Psychopharmacology, 23(2), 90-96.

David, S. P., Munafò, M. R., Johansen-Berg, H., Smith, S. M., Rogers, R. D., Matthews, P. M., \& Walton, R. T. (2005). Ventral striatum/nucleus accumbens activation to smoking-related pictorial cues in smokers and nonsmokers: a functional magnetic resonance imaging study. Biological Psychiatry, 58(6), 488-94.

de Ruiter, M. B., Oosterlaan, J., Veltman, D. J., van den Brink, W., \& Goudriaan, A. E. (2012). Similar hyporesponsiveness of the dorsomedial prefrontal cortex in problem gamblers and heavy smokers during an inhibitory control task. Drug and Alcohol Dependence, 121(1-2), 819.

Due, D. L., Huettel, S. A., Hall, W. G., \& Rubin, D. C. (2002). Activation in mesolimbic and visuospatial neural circuits elicited by smoking cues: evidence from functional magnetic resonance imaging. The American Journal of Psychiatry, 159(6), 954-60. 
Engelmann, J. M., Versace, F., Robinson, J. D., Minnix, J. a, Lam, C. Y., Cui, Y., ... Cinciripini, P. M. (2012). Neural substrates of smoking cue reactivity: a meta-analysis of fMRI studies. NeuroImage, 60(1), 252-62.

Feduccia, A. A., Chatterjee, S., \& Bartlett, S. E. (2012). Neuronal nicotinic acetylcholine receptors: neuroplastic changes underlying alcohol and nicotine addictions. Frontiers in Molecular Neuroscience, 5, 83.

Field, M., \& Cox, W. M. (2008). Attentional bias in addictive behaviors: a review of its development, causes, and consequences. Drug and Alcohol Dependence, 97(1-2), 1-20.

Field, M., Duka, T., Tyler, E., \& Schoenmakers, T. M. (2009). Attentional bias modification in tobacco smokers. Nicotine \& Tobacco Research, 11(7), 812-22.

Field, M., Marhe, R., \& Franken, I. H. A. (2014). The clinical relevance of attentional bias in substance use disorders. CNS Spectrums, 19(03), 225-230.

Field, M., Munafò, M. R., \& Franken, I. H. A. (2009). A meta-analytic investigation of the relationship between attentional bias and subjective craving in substance abuse. Psychological Bulletin, 135(4), 589-607.

Fiore, M. C., Jaén, C. R., Baker, T. B., Bailey, W. C., Benowitz, N. L., Curry, S. J., ... Wewers, M. E. (2008). Treating tobacco use and dependence: 2008 update. Rockville(MD): US Department of Health and Human Services 2008.

Franken, I. H. A. (2003). Drug craving and addiction: integrating psychological and neuropsychopharmacological approaches. Progress in Neuropsychopharmacology and Biological Psychiatry, 27(4), 563-79.

Franklin, T. R., Wang, Z., Wang, J., Sciortino, N., Harper, D., Li, Y., ... Childress, A. R. (2007). Limbic activation to cigarette smoking cues independent of nicotine withdrawal: a perfusion fMRI study. Neuropsychopharmacology, 32(11), 2301-2309.

Goldstein, R. Z., \& Volkow, N. D. (2011). Dysfunction of the prefrontal cortex in addiction: neuroimaging findings and clinical implications. Nature Reviews. Neuroscience, 12(11), 65269.

Hester, R., \& Luijten, M. (2013). Neural correlates of attentional bias in addiction. CNS Spectrums, (August), 1-8.

Hogarth, L. C., Mogg, K., Bradley, B. P., Duka, T., \& Dickinson, A. (2003). Attentional orienting towards smoking-related stimuli. Behavioural Pharmacology, 14, 153-160.

Hukkanen, J., Jacob, P., \& Benowitz, N. L. (2005). Metabolism and disposition kinetics of nicotine. Pharmacological Reviews, 57(1), 79-115.

Janes, A. C., Pizzagalli, D. A., Richardt, S., Frederick, B. deB., Chuzi, S., Pachas, G., ... Kaufman, 
M. J. (2010). Brain reactivity to smoking cues prior to smoking cessation predicts ability to maintain tobacco abstinence. Biological Psychiatry, 67(8), 722-729.

Janes, A. C., Pizzagalli, D. A., Richardt, S., Frederick, B. deB., Holmes, A. J., Sousa, J., ... Kaufman, M. J. (2010). Neural substrates of attentional bias for smoking-related cues: an FMRI study. Neuropsychopharmacology, 35(12), 2339-2345.

Jiloha, R. C. (2010). Biological basis of tobacco addiction: Implications for smoking-cessation treatment. Indian Journal of Psychiatry, 52(4), 301-7.

Kerst, W. F., \& Waters, A. J. (2014). Attentional retraining administered in the field reduces smokers' attentional bias and craving. Health Psychology, 33(10), 1232-40.

Koob, G. F. (2009). Dynamics of neuronal circuits in addiction: reward, antireward, and emotional memory. Pharmacopsychiatry, 42 Suppl 1(S 01), S32-41.

Koob, G. F. (2013). Negative reinforcement in drug addiction: The darkness within. Current Opinion in Neurobiology, 23(4), 559-563.

Koob, G. F., \& Volkow, N. D. (2010). Neurocircuitry of addiction. Neuropsychopharmacology, 35(1), 217-38.

Lopes, F. M., Pires, A. V., \& Bizarro, L. (2014). Attentional bias modification in smokers trying to quit: a longitudinal study about the effects of number of sessions. Journal of Substance Abuse Treatment, 47(1), 50-7.

Luijten, M., Veltman, D. J., Hester, R., Smits, M., Nijs, I. M. T., Pepplinkhuizen, L., \& Franken, I. H. A. (2013). The role of dopamine in inhibitory control in smokers and non-smokers: a pharmacological fMRI study. European Neuropsychopharmacology, 23(10), 1247-56.

Luijten, M., Veltman, D. J., van den Brink, W., Hester, R., Field, M., Smits, M., \& Franken, I. H. A. (2011). Neurobiological substrate of smoking-related attentional bias. NeuroImage, 54(3), 2374-81.

Martin-Soelch, C. (2013). Neuroadaptive changes associated with smoking: structural and functional neural changes in nicotine dependence. Brain Sciences, 3(1), 159-76.

McBride, D., Barrett, S. P., Kelly, J. T., Aw, A., \& Dagher, A. (2006). Effects of expectancy and abstinence on the neural response to smoking cues in cigarette smokers: an fMRI study. Neuropsychopharmacology, 31, 2728-2738.

McHugh, R. K., Murray, H. W., Hearon, B. A., Calkins, A. W., \& Otto, M. W. (2010). Attentional bias and craving in smokers: the impact of a single attentional training session. Nicotine \& Tobacco Research, 12(12), 1261-4.

Mogg, K., \& Bradley, B. P. (2002). Selective processing of smoking-related cues in smokers: manipulation of deprivation level and comparison of three measures of processing bias. 
Journal of Psychopharmacology, 16(4), 385-392.

Mogg, K., Field, M., \& Bradley, B. P. (2005). Attentional and approach biases for smoking cues in smokers: An investigation of competing theoretical views of addiction. Psychopharmacology, 180, 333-341.

Nestor, L., McCabe, E., Jones, J., Clancy, L., \& Garavan, H. (2011). Differences in "bottom-up" and "top-down" neural activity in current and former cigarette smokers: Evidence for neural substrates which may promote nicotine abstinence through increased cognitive control. NeuroImage, 56(4), 2258-2275.

Noël, X., Brevers, D., \& Bechara, A. (2013). A neurocognitive approach to understanding the neurobiology of addiction. Current Opinion in Neurobiology, 23, 632-638.

Nutt, D., King, L. A., Saulsbury, W., \& Blakemore, C. (2007). Development of a rational scale to assess the harm of drugs of potential misuse. Lancet, 369(9566), 1047-53.

Pessoa, L., \& Ungerleider, L. G. (2004). Neuroimaging studies of attention and the processing of emotion-laden stimuli. Progress in Brain Research, 144, 171-182.

Peuker, A. C., \& Bizarro, L. (2013). Attentional avoidance of smoking cues in former smokers. Journal of Substance Abuse Treatment.

Piasecki, T. M., Richardson, A. E., \& Smith, S. M. (2007). Self-monitored motives for smoking among college students. Psychology of Addictive Behaviors, 21(3), 328-337.

Powell, J., Dawkins, L., West, R., Powell, J., \& Pickering, A. (2010). Relapse to smoking during unaided cessation: clinical, cognitive and motivational predictors. Psychopharmacology, 212(4), 537-49.

Rigotti, N. A. (2013). Smoking cessation in patients with respiratory disease: existing treatments and future directions. Lancet. Respiratory Medicine, 1(3), 241-50.

Robinson, T. E., \& Berridge, K. C. (2008). The incentive sensitization theory of addiction: some current issues. Philosophical Transactions of the Royal Society of London. Series B, Biological Sciences, 363(July), 3137-3146.

Rosecrans, J. A., \& Karan, L. D. (1993). Neurobehavioral mechanisms of nicotine action: role in the initiation and maintenance of tobacco dependence. Journal of Substance Abuse Treatment, $10(2), 161-70$.

Rubinstein, M. L., Luks, T. L., Moscicki, A. B., Dryden, W., Rait, M. ., \& Simpson, G. V. (2010). Smoking-related cue-induced brain activation in adolescent light smokers. Journal of Adolescent Health, 48(1), 7-12.

Shiffman, S., Dunbar, M. S., Scholl, S. M., \& Tindle, H. A. (2012). Smoking motives of daily and non-daily smokers: a profile analysis. Drug and Alcohol Dependence, 126(3), 362-8. 
Smolka, M. N., Buhler, M., Klein, S., Zimmermann, U., Mann, K., Heinz, A., \& Braus, D. F. (2006). Severity of nicotine dependence modulates cue-induced brain activity in regions involved in motor preparation and imagery. Psychopharmacology, 184(3-4), 577-588.

Spiegelhalder, K., Jähne, A., Kyle, S. D., Beil, M., Doll, C., Feige, B., \& Riemann, D. (2011). Is Smoking-related Attentional Bias a Useful Marker for Treatment Effects? Behavioral Medicine, 37(1), 26-34.

Volkow, N. D. (2002). Role of Dopamine, the Frontal Cortex and Memory Circuits in Drug Addiction: Insight from Imaging Studies. Neurobiology of Learning and Memory, 78(3), 610 624.

Volkow, N. D., Fowler, J. S., \& Wang, G.-J. (2004). The addicted human brain viewed in the light of imaging studies: brain circuits and treatment strategies. Neuropharmacology, 47 Suppl 1, 3-13.

Volkow, N. D., Wang, G. J., Tomasi, D., \& Baler, R. D. (2013). Unbalanced neuronal circuits in addiction. Current Opinion in Neurobiology, 23(4), 639-648.

Watanabe, M., Hikosaka, K., Sakagami, M., \& Shirakawa, S. (2002). Coding and Monitoring of Motivational Context in the Primate Prefrontal Cortex. Journal of Neuroscience, 22(6), 23912400 .

Waters, A. J., Shiffman, S., Bradley, B. P., \& Mogg, K. (2003). Attentional shifts to smoking cues in smokers. Addiction, 98, 1409-1417.

Waters, A. J., Shiffman, S., Sayette, M. A., Paty, J. A., Gwaltney, C. J., \& Balabanis, M. H. (2003). Attentional bias predicts outcome in smoking cessation. Health Psychology, 22(4), 378-87.

Wilson, S. J., Sayette, M. A., \& Fiez, J. A. (2004). Prefrontal responses to drug cues: a neurocognitive analysis. Nature Neuroscience, 7(3), 211-4.

World Health Organization. (2015). WHO | Tobacco Factsheet. Geneva: World Health Organization. 
Neural and cognitive determinants of smoking addiction and cessation 


\section{CHAPTER 1}

Treatment with a nicotine vaccine does not lead to changes in brain activity during smoking cue exposure or a working memory task.

Havermans, A., Vuurman, E.F.P.M., van den Hurk, J., Hoogsteder P., \& van Schayck C.P. (2014). Treatment with a nicotine vaccine does not lead to changes in brain activity during smoking cue exposure or a working memory task. Addiction, 109, 1260-1267. 


\begin{abstract}
The aim of this randomized, placebo controlled parallel-group, repeated measures trial was to demonstrate that immunization attenuates nicotinic stimulation of the brain. In addition we aim to elucidate its effects on brain and behavioural responses during exposure to smoking cues and a working memory task. To achieve this, forty-eight smoking male volunteers were randomized to receive five injections with either $400 \mu \mathrm{g} / \mathrm{ml}$ of the 3 'aminomethylnicotine P. aeruginosa r-Exoprotein conjugated vaccine or placebo over a period of 20 weeks. Subjects were tested on two occasions; once after a nicotine challenge and once after a placebo challenge and were asked to refrain from smoking 10 hours before testing. Reaction times and accuracies were recorded during an n-back task. Moreover, regional blood oxygenated level dependent (BOLD) response was measured during this task and during smoking cue exposure. Greater activation was found in response to smoking cues compared to neutral cues in bilateral trans-occipital sulcus ( $p<.005$ uncorrected). There was no difference in brain activity to smoking cues between the treatment groups and no effects of acute nicotine challenge were established. For the n-back task we found working memory load-sensitive increases in brain activity in several frontal and parietal areas $(\mathrm{p}$ <.0025). However, no effects of immunization or nicotine challenge were observed. In conclusion, no significant effects of immunization on brain activity in response to a nicotine challenge were established. Therefore this vaccine is not likely to be an effective aid in smoking cessation
\end{abstract}




\section{Introduction}

Implicated in approximately 6 million deaths per year worldwide, tobacco use continues to be the leading cause of preventable death (World Health Organization, 2013). Although most smokers are well aware of the health consequences, and want to quit, they have difficulty doing so (Persoskie \& Nelson, 2013). Therapies and pharmacological treatments have been developed to aid smokers in cessation, however long term success rates remain low as only $15-30 \%$ of smokers stay abstinent for at least one year after treatment (Fiore et al., 2008). Since the vast majority of those who attempt to quit will fail, the need for better approaches to smoking cessation is clear and urgent.

A potential new treatment approach in smoking cessation and relapse prevention is active nicotine immunization. Anti-nicotine vaccines have been developed by chemically linking the (non-immunogenic) nicotine molecule to a larger carrier protein and an adjuvant that induces an immune response. The elicited antibodies bind to nicotine in the bloodstream, forming a complex which is too large to cross the blood-brain barrier. Successive doses of an anti-nicotine vaccine raise antibody levels in the bloodstream and are thought to gradually decrease the amount of nicotine reaching the brain (Cerny \& Cerny, 2009).

Normally, nicotine binds in the brain to nicotinic acetylcholine receptors (nAChR's), which results in the release of a variety of neurotransmitters. Most importantly, it causes the release of dopamine from mesolimbic neurons, which is thought to be critical to nicotine's reinforcing effects. Moreover, repetitive exposure to nicotine results in the development of tolerance to its effects and causes upregulation of nAChR's. During smoking cessation, these nAChR's become unoccupied and dopamine is cut off, as a result people can experience intense cravings and withdrawal symptoms (Benowitz, 2008a, 2008b). As a consequence of immunization it is expected that with decreasing amounts of nicotine reaching the brain also dopamine release declines, reducing the positive reinforcement of smoking. Ultimately, this should diminish craving and withdrawal symptoms as well, making it less difficult for ex-smokers to stay abstinent (Cerny \& Cerny, 2009). Recently it has been demonstrated that treatment with 3 'AmNic-rEPA vaccine leads to a $12.5 \%$ reduction in nicotine receptor binding (Esterlis et al., 2013). This finding supports the notion that the vaccine prevents a certain amount of nicotine from entering the brain. Moreover, immunization was associated with significant reductions in cigarette use and craving (Esterlis et al., 2013).

However, tobacco addiction is not only maintained by the pharmacological effects of nicotine. The urge to smoke is often triggered by environmental cues that have been associated with the rewarding effects of nicotine (Benowitz, 2008b). For long-term smokers these environmental cues can become extremely salient and attention-grabbing, especially 
during periods of abstinence (Field \& Cox, 2008). Functional imaging studies have shown that exposure to such smoking cues (contrasted to neutral cues) results in increased activation of a broad range of brain areas, mostly located in the extended visual system of the occipital, inferior temporal, and posterior parietal lobes, and in the cingulate gyrus and prefrontal cortex (Engelmann et al., 2012). Furthermore, regions in the lingual gyrus (LG) and superior frontal gyrus (SFG) were more consistently activated in deprived smokers than in non-deprived smokers when viewing smoking cues compared to neutral ones (Engelmann et al., 2012). This finding supports the notion of more resources being allocated to the processing of smoking cues when smokers are deprived of nicotine. Since immunization with an anti-nicotine vaccine will lead to a decreased supply of nicotine to the brain it might also lead to smoking cues becoming more salient. It is thus important to understand what the consequences of immunization are for cue induced craving as this could have a negative influence on treatment outcome.

Another reason for smoking that smokers provide is that it improves their concentration or performance on certain tasks (Benowitz, 2008a). Indeed several studies have demonstrated beneficial effects of nicotine on cognitive tasks (Kumari et al., 2003; Newhouse, Potter, \& Singh, 2004). One of the cognitive functions known to be positively influenced by nicotine is working memory, as several studies have demonstrated that nicotine improved performance on behavioural measures and caused increased activity in several brain areas, among which the anterior cingulate and superior frontal and -parietal cortices (Ernst et al., 2001; Kumari et al., 2003). In accordance, smoking abstinence has been associated with decreased accuracy rates and increased reaction times on the same task (Mendrek et al., 2006; Myers, Taylor, Moolchan, \& Heishman, 2008; Xu et al., 2005), accompanied by increases in task-related activation in the dorsolateral prefrontal cortex (dlPFC) (Xu et al., 2005, 2006). As immunization leads to reduced amounts of nicotine reaching the brain after smoking it should also be considered what its consequences are for cognitive functioning. In the end cognitive difficulties after smoking cessation could lead to relapses.

In conclusion, tobacco addiction is multifaceted and when evaluating immunization as a treatment its effects on all these facets should be considered. Exposing the broader range of effects of immunization is essential for a good interpretation of clinical results and could thereby contribute to improvement of treatment strategies. Therefore our study made use of functional magnetic resonance imaging (fMRI) to elucidate brain activity of smokers treated with the nicotine conjugate vaccine NicVAX and non-treated smokers during exposure to smoking cues and a working memory task. Our first aim was to confirm the pharmacological mechanism of this vaccine by demonstrating nicotine mediated differences in brain activity between vaccinated and non-vaccinated smokers. Because we 
mainly expected differences between the treatment groups after consumption of nicotine we tested all participants on two occasions, once after administration of nicotine and once after a placebo.

Moreover, it was of high interest to us to examine whether and how other facets of tobacco addiction were affected by aberrant nicotine availability in the brain. Therefore the secondary aim was to elucidate the effects of nicotine immunization on brain responses to smoking cue exposure. We expected that over the course of treatment, the participants in the immunized group would have become desensitized for smoking cues as a result of the decreasing associated nicotine-reward. Therefore it was hypothesized that the notimmunized participants would show increased activity in the LG and SFG (Engelmann et al., 2012) as compared to the immunized participants. Moreover, administrating nicotine should diminish the differences between the groups.

In addition, we wanted to find out the influence of immunization and thus reduced nicotine availability on a working memory task. In this case it was hypothesized that the nicotine challenge would improve behavioural results, only in the not immunized group. Also we expected increases in task-related brain activity in frontal and parietal regions after the nicotine challenge in the non-vaccinated group compared to the vaccinated group (Kumari et al., 2003; Xu et al., 2005, 2006). 


\section{Methods}

\section{Participants}

A total of 48 male participants were included in this study. Suitable candidates were recruited through local and online newspaper advertisements. Participants were all righthanded smokers who reported smoking $\geq 10$ cigarettes a day for at least one year. Participant demographics are displayed in table 1. Main exclusion criteria were history of physical or mental illness, use of psychotropic medication, history of drug or alcohol abuse, or contraindications for the MRI scanner. Before inclusion, participants were informed about the aims, procedure and risks of the study and gave written informed-consent. Participants were financially compensated for their participation and travel costs. The study was approved by the Dutch Central Committee on Research Involving Human Subjects (CCMO).

\section{Experimental Procedure}

Participants were randomly assigned to receive 5 subsequent injections with either 400 $\mu \mathrm{g} / \mathrm{ml}$ of the 3 'aminomethylnicotine $P$. aeruginosa r-Exoprotein conjugate vaccine (NicVax, Nabi Biopharmaceuticals) $(\mathrm{N}=28)$ or a placebo $(\mathrm{N}=20)$. Inoculations were scheduled on days $0,28,56,84$ and 112 and were administered in the alternating deltoid muscle. During the final inoculation visit, participants practised the behavioural task and were familiarized with the MRI environment in a mock MRI scanner. Actual fMRI scanning and behavioural testing were planned 18 and 21 days after the last inoculation, when antibody titres were expected to be near maximal. Participants were instructed to refrain from smoking and taking stimulating food or drinks (i.e. coffee, chocolate) 10 hours before testing. Smoking abstinence was verified by exhaled carbon monoxide measurement; $\mathrm{CO}$ levels were $50 \%$ or more below baseline. In addition smoking behaviour of the preceding week was assessed by means of a questionnaire. The test procedure was repeated on two days with an interval of at least one week. Participants received two pieces of mint flavoured nicotine gum $2 \mathrm{mg}$ (Nicotinell®) during one session and two pieces of sugar free, mint flavoured regular chewing gum, during the other session, which were matched for shape and size. Challenge order was single blind and randomized. After the last test session (approx. day 140) serum samples were taken and sent to Nabi Biopharmaceuticals to assess anti-nicotine $\operatorname{IgG}$ antibody concentrations, using Enzyme linked immunosorbet assay (Voller, 1978).

\section{Imaging data acquisition}

Functional and anatomical images were acquired with a 3 Tesla Siemens Magnetom Allegra head scanner (Siemens Medical Systems, Erlangen, Germany). Blood-oxygen- 
level-dependent (BOLD) functional images were collected for 32 contiguous slices (voxelsize $=3.5 \times 3.5 \times 3.5 \mathrm{~mm}$.) covering as much as possible the entire brain. A standard gradient echo planar imaging (EPI) sequence was applied ( $\mathrm{TR}=2 \mathrm{~s}, \mathrm{TE}=30 \mathrm{~ms}$, matrix size $=64 \times 64)$. Whole brain structural images were acquired using a $1 \times 1 \times 1 \mathrm{~mm}$ resolution $\mathrm{T} 1$ weighted $\mathrm{ADNI}$ sequence $(\mathrm{TR}=2250 \mathrm{~ms}, \mathrm{TE}=2.6 \mathrm{~ms})$. Participants were lying on the scanner bed in supine position with their heads fixated with foam pads. Stimuli were projected on a screen at the back of the scanner bore and were visible to the participants through a mirror attached to the head coil. Responses were made with handheld button boxes. Stimulus presentation was accomplished with E-prime ${ }^{\circledR}$ (Psychology Software Tools, Inc. Sharpsburg, PA) and synchronised to the MR data acquisition.

\section{Cue exposure}

Participants were presented with 20 blocks of 3 colour photos with smoking related or neutral content. The smoking related images $(\mathrm{n}=30)$ included people smoking (only the heads or mouths), hands holding a cigarette and cigarettes in ashtrays or in the pack. Neutral images $(n=30)$ were matched for shape of the object and general content, and included e.g. a person brushing his teeth, a hand holding a screwdriver, chopsticks on a bowl and pencils in a pack. Each stimulus was presented 6 seconds, totalling 18 seconds per set. Stimulus sets were presented in random order with intervals of 18 seconds, during which a fixation cross was visible.

\section{$N$-Back working memory task}

A four-step parametric letter version of the n-back task was employed (Carlson et al., 1998; Kumari et al., 2003). The task consisted of four conditions with increasing demands on working memory. In the 1-back condition participants indicated for each letter whether it was identical to the previous one, by pressing a target key in case it was the same and otherwise pressing a non-target key. For the 2- and 3-back conditions the procedure was similar and participants indicated for each letter whether it was the same as the letter that had been displayed respectively two and three letters back. The final condition was a nonworking memory demanding control task in which participants indicated for each letter whether it was an ' $x$ ' or not. Letters were presented centrally on a black screen until the participant responded, with a maximum duration of 1 second per letter. The inter stimulus interval (ISI) was adjusted after each stimulus, so that stimulus duration and ISI together always added up to two seconds (1 TR). Four blocks of each N-back condition were presented, each containing 4 (19\%) targets and 17 non-targets (Two blocks contained 3 targets and two blocks contained 5). Mean reaction time (RT) and accuracy were recorded for all 4 memory load (Wmload) conditions. 


\section{Behavioral data analysis}

Statistical analysis of the N-back task was performed using IBM SPSS Statistics version 19. Repeated measures analyses of variance (ANOVA) were conducted with Wmload (find X, 1-, 2-, and 3-back) and Gumtype (nicotine or placebo) as within-subject independent conditions and Treatment (Active vaccine or Placebo) as a between subjects factor. Separate analyses were conducted for the dependent variables Reaction Time and Accuracy.

\section{Imaging data analysis}

Imaging data was pre-processed and analyzed using Brainvoyager QX version 2.4 (Brain Innovation, Maastricht, The Netherlands). The first two volumes of all functional runs were discarded due to T1 saturation. The rest of the functional data was corrected for slice scantime differences and 3D head motion using 3 translation and 3 rotation parameters. Subsequently, linear trends and low frequency temporal drifts were removed from the data using a high-pass filter. After the pre-processing, functional data were co-registered to the anatomical volume and transformed to Talairach space. To further reduce the effects of motion-related variability, a custom Matlab R2012b (Mathworks Inc, Natick, MA) algorithm was applied that first (per voxel) computed which volumes had a brightness value that yielded 4 times the standard deviation compared to the mean signal of that voxel. Then, it compared all voxels and determined for which volumes the majority of voxels were labelled as affected by 'excessive' motion. These volumes were deleted from the full dataset of that subject.

The analyses were performed using a whole-brain random effects (RFX) general linear model (GLM) with subject as random variable. For each stimulus condition a time course was modeled by convolving a boxcar function representing the times of onset and duration of each stimulus block with a canonical (double gamma) hemodynamic response function (HRF). Then a design matrix was created for each participant individually, using such a predictor for each condition. For the cue task in order to test for the effect of the smoking cues, we computed the contrast between the smoking cue condition and the neutral cue condition. Subsequently the difference in smoking cue reactivity (smoking greater than control) between the two gum types [placebo > nicotine] and between the treatment groups was examined. To find task related effects in the n-back task, responses to the 1-back condition were subtracted from those to the 2-back condition. Subsequently voxelwise whole brain interaction analyses of (Wmload x Treatment), (Wmload x Gumtype), (Gumtype x Treatment) and (Wmload x Gumtype x Treatment) were performed. Resulting contrast maps were statistically corrected for multiple comparisons (FDR corrected at $q=$ 
0.05). A white matter reconstruction of both hemispheres of a representative participant was made and consecutively inflated for visualization of the data. 


\section{Results}

\section{Participants}

Over the course of the study 13 participants have dropped out (6 received active treatment, 7 placebo) Dropouts were due to planning issues or distress in the MRI scanner and could not be attributed to participant characteristics such as degree of nicotine dependence. In addition, two participants from the active vaccine group were excluded from analyses; one due to an invalid informed consent and the other failed to show a considerable antibody count. Thus, for the behavioural analysis 20 participants were included in the active treatment group and 13 in the placebo group (see table 1). The mean amount of cigarettes smoked by the participants in the week prior to testing did not significantly change after immunization $(\mathrm{p}=.27)$

Table 1. Participant demographics at baseline (Mean \pm SD)

\begin{tabular}{llllc}
\hline & $\begin{array}{l}\text { NicVAX } \\
\mathrm{N}=20^{*}\end{array}$ & $\begin{array}{l}\text { Placebo } \\
\mathrm{N}=13^{*}\end{array}$ & \multirow{2}{*}{$t$} & $p$ \\
\hline Age (years) & $33.4(7.1)$ & $28.8(7.1)$ & $(-1.492)$ & .146 \\
Nr. of cigarettes smoked in the past week & $125.9(99.9)$ & $129.7(56.2)$ & .167 & .869 \\
Duration of regular smoking (months) & $162.1(97.9)$ & $134(92.9)$ & $(-.82)$ & .418 \\
FTND $^{* *}$ & $4.2(1.8)$ & $4.7(2.7)$ & .628 & .534 \\
Expired CO $(\mathrm{ppm})$ & $30.4(16.8)$ & $25.2(11.9)$ & $(-.976)$ & .336 \\
\hline
\end{tabular}

* Numbers based on analysis with highest N (= N-back behavioural data) ** FTND, Fagerström Test for Nicotine Dependence, range of values 0 - 10 (higher scores indicate a higher level of dependence)

\section{Antibody response}

The mean antibody level for the active treatment group was $43.83 \mu \mathrm{g} / \mathrm{mL}(\mathrm{SD}=17.84)$ and all but one of the subjects reached a level above $25 \mu \mathrm{g} / \mathrm{mL}$. Plasma antibody levels in the active treatment group were significantly higher $\left(\mathrm{t}_{(32)} ; \mathrm{p}<0.001\right)$ than those of the placebo group, as none of the participants in the latter group showed a detectable antibody level.

\section{Behavioural results}

The behavioural results of the n-back task are shown in figure 1 and 2. Overall, participants showed significantly lower RTs over the highest memory- load condition (3-back) compared to all the other conditions $\left(\mathrm{F}_{(3)}=13.16 ; \mathrm{p}<.001\right)$. Mean response accuracies 
decreased significantly with each increase in wm-load $\left(\mathrm{F}_{(3)}=69.78 ; \mathrm{p}<.001\right)$. No main effects of Treatment and Gumtype were found on either response latencies or accuracy. However, there was a significant interaction between Treatment and Wmload $\left(\mathrm{F}_{(3)}=3.66 ; \mathrm{p}\right.$ $<.05)$ on response accuracy. Post hoc analysis revealed that the mean accuracy of participants in the NicVax group was higher than that of participants in the placebo group, only for the control condition that didn't demand working memory. For the other task conditions, the accuracies of the placebo group were higher.
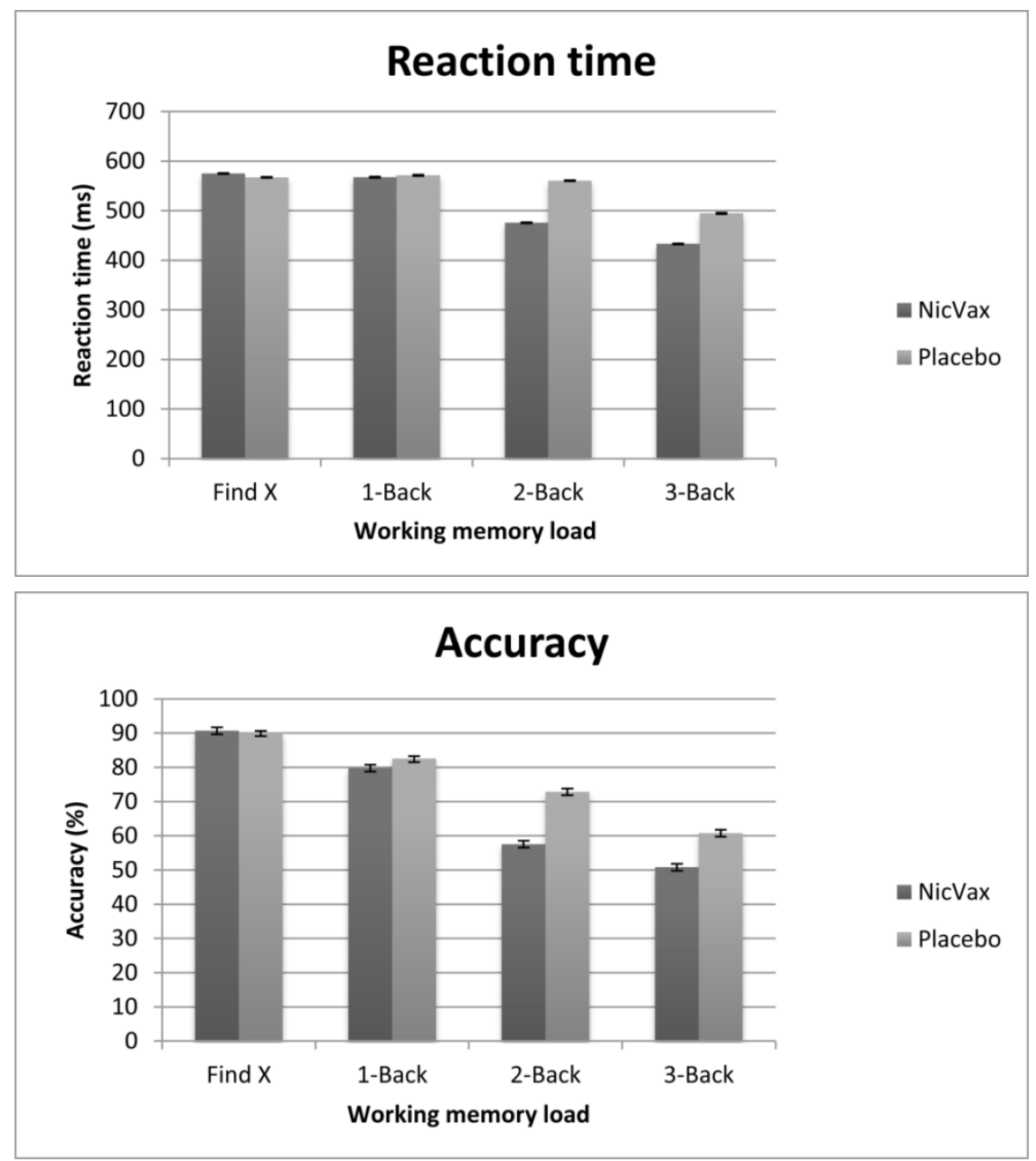

Figure 1 and 2. Behavioral results of the $N$-Back task (N=33). Mean reaction times (above) and accuracies (below) for each level of the task and each treatment group separately. 


\section{Imaging results}

Cue exposure

Three participants were excluded from analysis due to malfunction of technical equipment. This led to a number of 19 participants in the active treatment group and 11 in the placebo group. Across both treatment conditions participants exhibited greater BOLD-response to smoking cues compared to neutral cues in medial occipital areas $\left(\mathrm{t}_{(29)}>3.04 ; p<.005\right.$ uncorrected, see figure 3), however this effect did not survive correction for multiple comparisons. There was no difference in brain activity to smoking cues between the treatment groups and no effects of acute nicotine challenge were established.

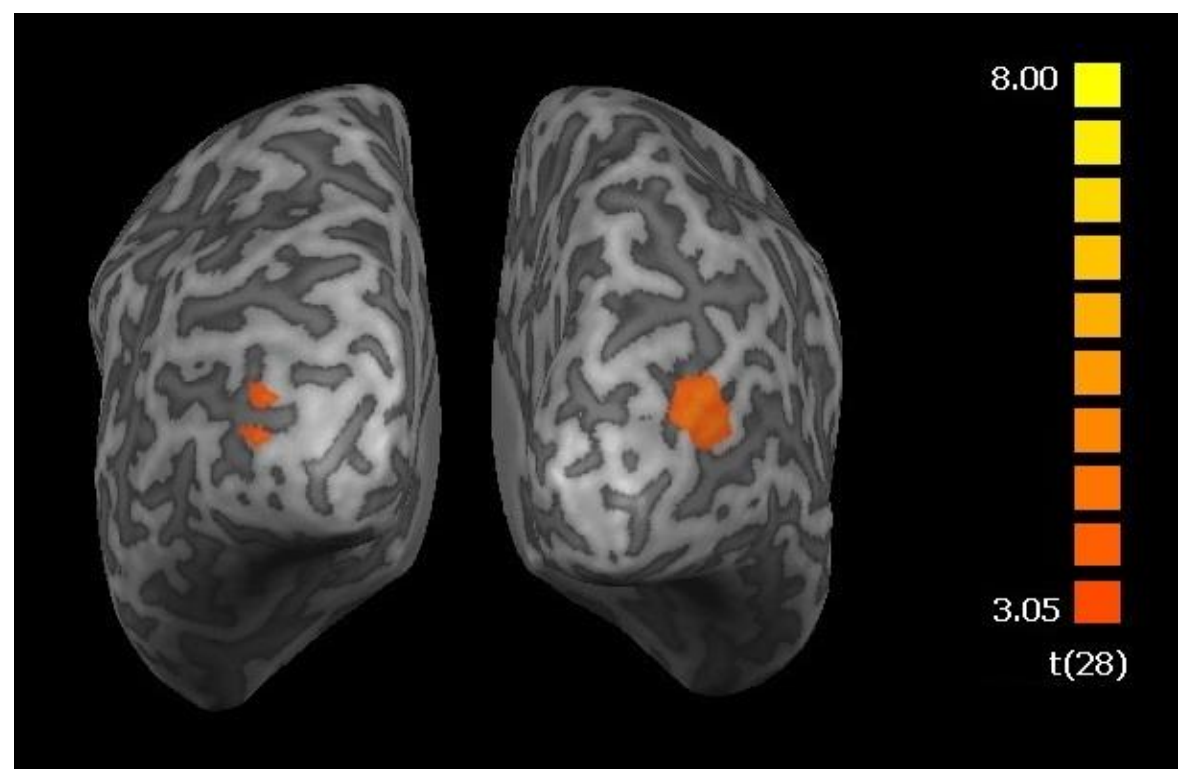

Figure 3. Smoking Cue Reactivity (N=30). Bilateral activation at trans-occipital sulcus for smoking related > neutral cues at $p<.005$ uncorrected for multiple comparisons. Projected on an inflated individual cortex- reconstruction, posterior orientation.

N-Back working memory task

Three participants were excluded from analysis because of incomplete or missing data. Data from three other participants was discarded due to malfunction of the equipment; one of these participants had also been excluded from analysis in the cue exposure task. The final participant numbers were 17 in the active treatment group and 10 in the placebo group. Over all conditions, task related responses were found in a range of frontal and 
parietal areas, e.g. superior frontal gyrus, dorsolateral prefrontal cortex, insula, inferior parietal lobule and precuneus $\left(\mathrm{t}_{(26)}>3.34, \mathrm{p}<.0025\right.$. See figure 4$)$. No interacting effects of immunization or nicotine challenge could be demonstrated.

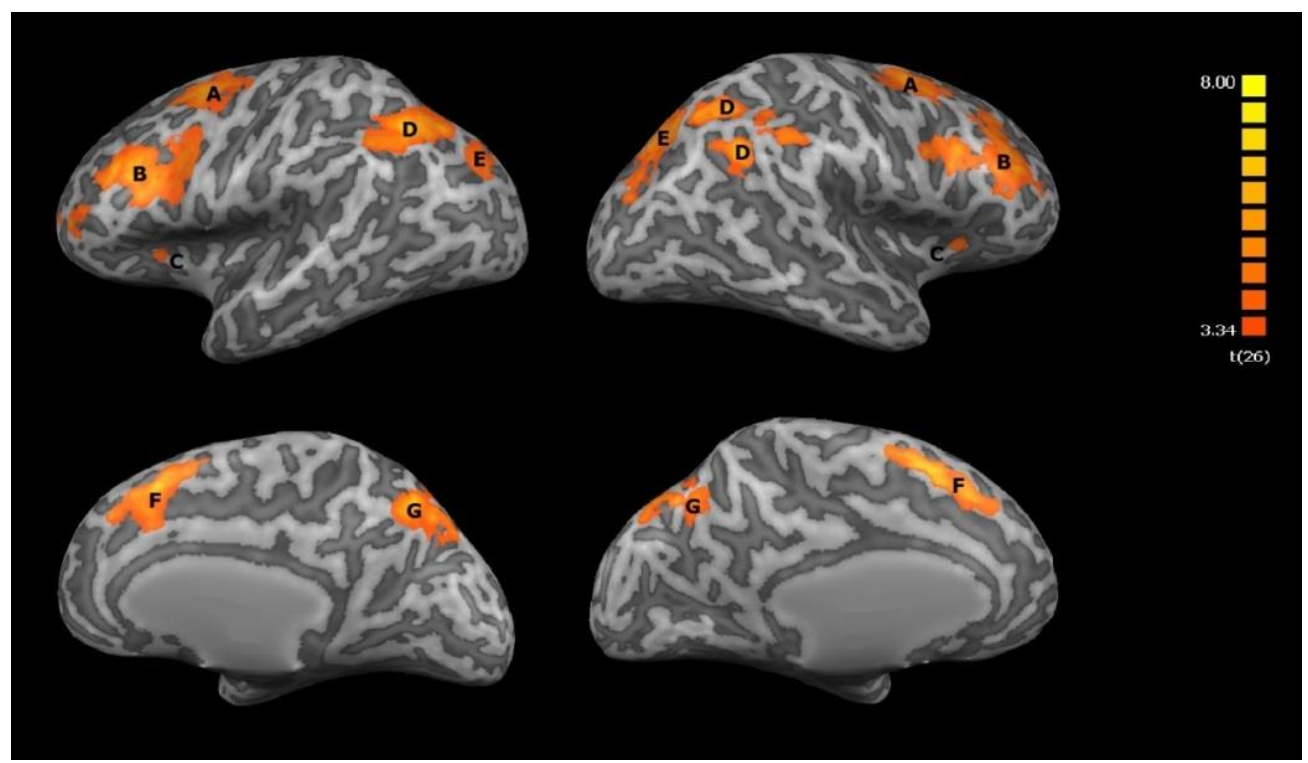

Figure 4. $\mathbf{N}$-Back task effect $(\mathbf{N}=27)$. Frontal and parietal areas which show higher activity during the 2 Back than the 1Back condition of the task. A: superior frontal gyrus, $\boldsymbol{B}$ : dorsolateral prefrontal cortex, $\boldsymbol{C}$ : insula, $\boldsymbol{D}$ : inferior parietal lobule, $\boldsymbol{E}$ : precuneus, $\boldsymbol{F}$ : (medial) superior frontal gyrus, $\boldsymbol{G}$ : (medial) precuneus. FDR-corrected at $q=.05 ; p<.0025$. Projected on an inflated individual cortex reconstruction. 


\section{Discussion}

The current study was designed to investigate the neurocognitive effects of a nicotine vaccine, which is thought to partly prevent nicotine from entering the brain. Our first aim was to confirm this mechanism by demonstrating differences in nicotine related brain activity between immunized and non-immunized participants by means of fMRI. Additionally we intended to elucidate the effects of this reduced nicotine availability on a couple of cognitive functions that have been shown to be involved in the maintenance of tobacco addiction (9-16). By administering a nicotine challenge or placebo to each participant prior to scanning them we expected to be able to directly compare the effects of nicotine on brain activity between vaccinated and non-vaccinated participants.

We found a clear increase in activity in bilateral trans-occipital sulcus in response to smoking related compared to neutral images, which is in accordance with previous studies that have reported increases in occipital areas of the extended visual system (Engelmann et al., 2012). However, these studies have also reported increased activity in response to smoking cues in many other areas. Additionally, the effect we found did not survive the correction for multiple comparisons. But most importantly no differences could be established between the treatment groups during smoking cue exposure after the placebo challenge, nor after administration of nicotine. On the N-back working memory task participants responded significantly faster to the 3-back condition. However, we suspect that this is the result of participants guessing for the right responses, as this condition may have been too difficult for some of them. We also found task related increased activity in several frontal and parietal regions, corresponding to findings of previous studies (Kumari et al., 2003; Xu et al., 2005, 2006). However, also for this task no effects of treatment or nicotine challenge were observed. Furthermore, immunized participants did not significantly reduce their weekly cigarette intake.

From these results we cannot conclude that the vaccine has successfully refrained nicotine from entering the brain and affecting regions known to be sensitive to nicotine uptake. Although the vaccine's proposed mechanism of action could thus not be supported by our data we may assume based on the study by Esterlis et al. (Esterlis et al., 2013) that immunization with NicVax did reduce the amount of nicotine reaching the brain. Therefore we pose that even though the immunization might have worked, its effect is not sufficient to influence ongoing processes in the brain in a way that can be demonstrated by means of fMRI or behavioural outcomes. This conclusion supports the results of recent clinical trials that have demonstrated poor efficacy of the vaccine (Fahim, Kessler, \& Kalnik, 2013; Hartmann-Boyce, Cahill, Hatsukami, \& Cornuz, 2012; Hoogsteder, Kotz, van Spiegel, Viechtbauer, \& van Schayck, 2014) and suggests that it is not likely to be an effective aid in smoking cessation. 
We should be careful interpreting our results however, as there could have been several other influences affecting them. First, it is surprising that we did not find any effects of the acute nicotine challenge, even in the placebo treatment group, as other studies did find changes in brain activity after administration of the same dose (Thiel \& Fink, 2008; Vossel, Thiel, \& Fink, 2008). An explanation could be that participants did not follow the instructions on proper use, such as intermittent chewing and "parking" the gum in their cheek, resulting in poor absorption. In line with this hypothesis it was occasionally observed that they did not like the taste of the gum and tried to avoid it, for instance by not chewing. Additionally, the fact that some participants disliked the flavour of the nicotine gum suggests that they were not completely blind to the challenge they received. This probably has not influenced our results as only a few participants actually recognized the unpleasant tasting gum as nicotine gum. Moreover participants had no knowledge about possible effects of nicotine, so it is not likely that this imperfect blinding has influenced their performance.

Second, it is possible that the antibody titres of several participants were suboptimal at the time of testing which could have led to an underestimation of the effects of immunization. This was the case as inoculations have been scheduled outside the preferred window per protocol (Ranging from 6 days earlier to 20 days later) on several occasions. Also some of the test sessions were scheduled outside the preferred window per protocol (ranging from 4 days earlier to 40 days later), due to planning difficulties of participants as well as technical problems with the MRI scanner. Eventually, taking into account the findings of Esterlis and colleagues (Esterlis et al., 2013), we assume that our titres could have been sufficient to reduce nicotine supply to the brain, but that even higher titres are necessary to affect BOLD response or behaviour.

Third, we observed excessive participant movement in the scanner, which confounded the fMRI signal of many participants to more or lesser degree. This relatively large amount of movement artefacts in our data was unexpected, although there are several explanations for it. First, all of our participants were unfamiliar with fMRI research. Even though we explained that it was very important to remain still inside the scanner, they were ignorant on how detrimental even a slight movement could be to the data. Moreover, many participants reported to feel overwhelmed or even frightened during the first moments of the scanning session, which could have led them to make small movements. We tried to prepare the participants to the feeling of being inside a MRI scanner during a practice run in a mock scanner. Yet the experience of being in a real operating scanner is still rather different from being inside the soundless fake scanner. Furthermore, while the practise run was very short (approx. 10 minutes), during the actual test-session participants were required to stay still in the scanner for as long as two hours (with one break half way). 
Participants' boredom and tiredness, especially at the end of the session, could also have contributed to their moving. And finally, extra difficulty to remain still could have arisen from the state of withdrawal the participants were in after being abstinent overnight. In fact, many participants showed typical restless and fidgety behaviour during the test sessions. Fortunately after removing all volumes that were heavily confounded by excessive motion we were able to use the data for analysis. Although this procedure was necessary, we acknowledge that cutting multiple volumes from a time course could have compromised the data to a certain extent as well.

A final limitation to our study might be the use of only male participants, as it is not certain whether the results can be generalized to the female population as well. However we needed to exclude females out of safety reasons, as teratogenicity of the vaccine had not been assessed before the start of our study. Not much attention has been paid to gender differences in pharmacological literature, but some studies suggest that smoking behaviour of women is influenced more by conditioned environmental cues than by nicotine itself. In accordance, nicotine replacement therapies seem less effective in women (Perkins, Donny, \& Caggiula, 1999). We could thus speculate that immunization would also be less effective in females than in males. However, since we were unable to find any effects of the vaccine in males, no less effect could have been demonstrated when studying females.

In the end, despite this relatively large amount of negative influences on the data, we did manage to find task-relevant effects which are consistent with existing literature. This indicates that the lack of nicotine and treatment effects in our data is not just caused by poor data quality or insufficient power. Still, power could have been further improved by using specific regions of interest (ROI) for analyses instead of the whole brain. This however would have required a more specific hypothesis about where the effect of the immunization would manifest itself in the brain. As this was the first time neurocognitive effects of a nicotine vaccine were studied, no exact expectations could be identified a priori. Therefore a more explorative approach analysing the whole brain was chosen.

In conclusion, no significant effects of treatment with NicVax on brain activity in response to a nicotine challenge have been established. This study was the first to use fMRI to elucidate whether a nicotine vaccine could actually influence brain functioning by restraining nicotine. We failed to confirm this and support the conclusions of clinical trials with the same vaccine. 


\section{Acknowledgements}

We would like to thank Anique van Dorp for setting up this study, Piet Portegijs for being the examining physician and Ilona van de Kolk and Pauline Horsten for administering the vaccines and moral support to the participants. We also thank Christoph Lynen and Marcel Loeffen for their helpful contributions during test sessions and data pre-processing. 


\section{References}

Benowitz, N. L. (2008a). Clinical pharmacology of nicotine: implications for understanding, preventing, and treating tobacco addiction. Clinical Pharmacology and Therapeutics, 83(4), 531-41.

Benowitz, N. L. (2008b). Neurobiology of nicotine addiction: implications for smoking cessation treatment. The American Journal of Medicine, 121(4 Suppl 1), S3-10.

Carlson, S., Martinkauppi, S., Rämä, P., Salli, E., Korvenoja, A., \& Aronen, H. J. (1998). Distribution of cortical activation during visuospatial n-back tasks as revealed by functional magnetic resonance imaging. Cerebral Cortex, 8(8), 743-52.

Cerny, E. H., \& Cerny, T. (2009). Vaccines against nicotine. Human Vaccines, 5(4), 200-5.

Engelmann, J. M., Versace, F., Robinson, J. D., Minnix, J. a, Lam, C. Y., Cui, Y., ... Cinciripini, P. M. (2012). Neural substrates of smoking cue reactivity: a metaanalysis of fMRI studies. NeuroImage, 60(1), 252-62.

Ernst, M., Matochik, J. A., Heishman, S. J., Van Horn, J. D., Jons, P. H., Henningfield, J. E., \& London, E. D. (2001). Effect of nicotine on brain activation during performance of a working memory task. Proceedings of the National Academy of Sciences of the United States of America, 98(8), 4728-4733.

Esterlis, I., Hannestad, J. O., Perkins, E., Bois, F., D’Souza, D. C., Tyndale, R. F., ... O'Malley, S. S. (2013). Effect of a nicotine vaccine on nicotine binding to $\beta 2 *_{-}$ nicotinic acetylcholine receptors in vivo in human tobacco smokers. The American Journal of Psychiatry, 170(4), 399-407.

Fahim, R. E. F., Kessler, P. D., \& Kalnik, M. W. (2013). Therapeutic vaccines against tobacco addiction. Expert Review of Vaccines, 12(3), 333-42.

Field, M., \& Cox, W. M. (2008). Attentional bias in addictive behaviors: a review of its development, causes, and consequences. Drug and Alcohol Dependence, 97(1-2), 120 .

Fiore, M. C., Jaén, C. R., Baker, T. B., Bailey, W. C., Benowitz, N. L., Curry, S. J., ... Wewers, M. E. (2008). Treating tobacco use and dependence: 2008 update. Rockville(MD): US Department of Health and Human Services 2008.

Hartmann-Boyce, J., Cahill, K., Hatsukami, D., \& Cornuz, J. (2012). Nicotine vaccines for smoking cessation. The Cochrane Database of Systematic Reviews, 8, CD007072. 
Hoogsteder, P. H. J., Kotz, D., van Spiegel, P. I., Viechtbauer, W., \& van Schayck, O. C. P. (2014). Efficacy of the nicotine vaccine 3'-AmNic-rEPA (NicVAX) co-administered with varenicline and counselling for smoking cessation: a randomized placebocontrolled trial. Addiction, 109(8), 1252-9.

Janes, A. C., Frederick, B. deB., Richardt, S., Burbridge, C., Merlo-Pich, E., Renshaw, P. F., ... Kaufman, M. J. (2009). Brain fMRI reactivity to smoking-related images before and during extended smoking abstinence. Experimental and Clinical Psychopharmacology, 17(6), 365-373.

Kumari, V., Gray, J. A., Ffytche, D. H., Mitterschiffthaler, M. T., Das, M., Zachariah, E., ... Sharma, T. (2003). Cognitive effects of nicotine in humans: an fMRI study. NeuroImage, 19(3), 1002-1013.

Mendrek, A., Monterosso, J., Simon, S. L., Jarvik, M., Brody, A., Olmstead, R., ... London, E. D. (2006). Working memory in cigarette smokers: comparison to nonsmokers and effects of abstinence. Addictive Behaviors, 31(5), 833-844.

Myers, C. S., Taylor, R. C., Moolchan, E. T., \& Heishman, S. J. (2008). Dose-related enhancement of mood and cognition in smokers administered nicotine nasal spray. Neuropsychopharmacology, 33(3), 588-98.

Newhouse, P. A., Potter, A., \& Singh, A. (2004). Effects of nicotinic stimulation on cognitive performance. Current Opinion in Pharmacology, 36-46.

Perkins, K. A., Donny, E., \& Caggiula, A. R. (1999). Sex differences in nicotine effects and self-administration: review of human and animal evidence. Nicotine \& Tobacco Research, 1(4), 301-15.

Persoskie, A., \& Nelson, W. L. (2013). Just blowing smoke? Social desirability and reporting of intentions to quit smoking. Nicotine \& Tobacco Research, 15(12), 2088 93.

Rubinstein, M. L., Luks, T. L., Moscicki, A. B., Dryden, W., Rait, M. ., \& Simpson, G. V. (2010). Smoking-related cue-induced brain activation in adolescent light smokers. Journal of Adolescent Health, 48(1), 7-12.

Thiel, C. M., \& Fink, G. R. (2008). Effects of the cholinergic agonist nicotine on reorienting of visual spatial attention and top-down attentional control. Neuroscience, 152, 381-390.

Voller, A. (1978). The enzyme-linked immunosorbent assay (ELISA) (theory, technique 
and applications). La Ricerca in Clinica E in Laboratorio, 8(4), 289-98.

Vossel, S., Thiel, C. M., \& Fink, G. R. (2008). Behavioral and neural effects of nicotine on visuospatial attentional reorienting in non-smoking subjects. Neuropsychopharmacology, 33(4), 731-8.

World Health Organization. (2013). WHO Report on the Global Tobacco Epidemic, 2013. Geneva: World Health Organization.

Xu, J., Mendrek, A., Cohen, M. S., Monterosso, J., Rodriguez, P., Simon, S. L., ... London, E. . (2005). Brain activity in cigarette smokers performing a working memory task: effect of smoking abstinence. Biological Psychiatry, 58(2), 143-150.

Xu, J., Mendrek, A., Cohen, M. S., Monterosso, J., Simon, S., Brody, A. L., ... London, E. D. (2006). Effects of acute smoking on brain activity vary with abstinence in smokers performing the N-Back task: a preliminary study. Psychiatry Research: Neuroimaging, 148(2-3), 103-109. 


\section{CHAPTER 2}

Nicotine deprivation elevates neural representation of smoking-related cues in object-sensitive visual cortex.

Havermans, A., van Schayck C.P., Vuurman, E.F.P.M., Riedel, W.J., \& van den Hurk, J. Nicotine deprivation elevates neural representation of smoking-related cues in objectsensitive visual cortex. (submitted). 


\begin{abstract}
In the current study, we use functional magnetic resonance imaging (fMRI) and multi-voxel pattern analysis (MVPA) to investigate whether tobacco addiction biases basic visual processing in favour of smoking-related images. We hypothesize that the neural representation of smoking-related stimuli in the lateral occipital complex (LOC) is elevated after a period of nicotine deprivation compared to a satiated state, but that this is not the case for object categories unrelated to smoking. Fourteen current smokers $(\geq 10$ cigarettes a day) were scanned in two sessions: once after 10 hours of nicotine abstinence and once after smoking ad libitum. Regional blood oxygenated level dependent (BOLD) response was measured while participants were presented with 24 blocks of 8 colour-matched pictures of cigarettes, pencils or chairs. The functional data were analysed through a pattern classification approach. In bilateral LOC clusters, the classifier was able to discriminate between patterns of activity elicited by visually similar smoking related (cigarettes) and neutral objects (pencils) above empirically estimated chance levels only during deprivation (mean $=61.5 \%$, mean permuted $=49.9 \%, \mathrm{p}=.009)$ but not during satiation (mean = $52.7 \%$, mean permuted $=49.9 \%$, ns.). For all other stimuli contrasts there was no difference in discriminability between the deprived and satiated conditions. In conclusion, the discriminability between smoking- and non-smoking visual objects was elevated in objectselective brain region LOC after a period of nicotine abstinence. This indicates that attention bias likely affects basic visual object processing.
\end{abstract}




\section{Introduction}

While tobacco addiction is often regarded as the direct result of the pharmacological effects of nicotine, there are various other processes involved as well. For instance, a critical role in the maintenance of tobacco addiction is reserved for the interaction between environment and corresponding neural events; i.e. drug related cues in the environment are paired with the rewarding physiological effects of nicotine. After repeated pairing and reinforcement of these cues they become highly salient, triggering the urge to smoke (Benowitz, 2010; Robinson \& Berridge, 2008). In this process, these motivationally relevant smoking cues have been shown to automatically and involuntarily capture the smoker's selective attention (Field \& Cox, 2008; Hogarth, Mogg, Bradley, Duka, \& Dickinson, 2003; Mogg \& Bradley, 2002). The resulting attention bias exhibited by smokers is associated with increased craving and has been implicated in maintaining addictive behaviour and provoking relapses (Austin \& Duka, 2012; Bradley, Mogg, Wright, \& Field, 2003; Janes, Pizzagalli, Richardt, Frederick, Chuzi, et al., 2010). Additionally, nicotine abstinence has been shown to increase subjective craving in response to smoking cues and enhance attention bias, making it even more difficult for (ex-) smokers to remain abstinent (Field, Mogg, \& Bradley, 2004; Gross, Jarvik, \& Rosenblatt, 1993; Sayette \& Hufford, 1994; Waters \& Feyerabend, 2000; Zack, Belsito, Scher, Eissenberg, \& Corrigall, 2001). Thus, even existing smoking cessation treatments will not be very effective as long as smokers are still automatically being attracted to these craving-eliciting cues. Accordingly, there is a need for new (additional) treatments targeting extinction of automatic responses to smoking cues. More knowledge about the exact underlying mechanisms can be essential in the process of developing those.

Functional imaging studies have shown that craving-provoking drug cues elicit a response in a network of frontal brain regions, mainly consisting of the amygdala, anterior cingulate cortex (ACC), dorsolateral prefrontal cortex (dIPFC), and orbitofrontal cortex (OFC) (Wilson, Sayette, \& Fiez, 2004). This is not surprising, since these areas are connected to the dopaminergic mesolimbic reward pathway which becomes activated in response to actual drug exposure (Brody, 2006). The amygdala is believed to enhance identification of emotionally salient stimuli (like conditioned drug cues) (Phillips, Drevets, Rauch, \& Lane, 2003), whereas the areas in the prefrontal cortex play a key role in the guidance of goal-directed and motivational behaviour. Specifically, the OFC is thought to integrate and modulate activity from several limbic areas (such as the amygdala) involved in reward processing (Volkow \& Fowler, 2000), and the dIPFC is implicated in regulatory processing and decision making (Watanabe, Hikosaka, Sakagami, \& Shirakawa, 2002). The ACC in addition, has been associated with conflict monitoring in the presence of competing response alternatives (Kerns et al., 2004). Reactivity of these areas to smoking cues may 
therefore reflect a process of deciding whether or not to resist the urge to smoke. Although findings are somewhat inconsistent across studies, increased activation in response to smoking cues is mostly reported in brain regions related to reward- and motivational processing as well as in the frontal and parietal attentional networks (Brody et al., 2007; Claus, Blaine, Filbey, Mayer, \& Hutchison, 2013; David et al., 2005; Franklin et al., 2007; Luijten et al., 2011; Rubinstein et al., 2010). Activation of these regions in response to smoking cues is evident, since it reflects the rewarding value of the cues and the motivational and attentional processes guiding drug-seeking behaviour (Engelmann et al., 2012).

Nonetheless, these areas are located relatively late in the pathway by which visual information is processed. That is, impulses are first transmitted from the primary visual cortex via extra striate cortex to higher-order visual association areas. From there, projections finally go to multimodal processing regions including the amygdala and prefrontal cortex (Tanaka, 1992). Interestingly, return connections of the amygdala to various levels of the ventral-stream are thought to enhance sensory processing of emotionally salient stimuli in the visual cortex (Amaral, Behniea, \& Kelly, 2003). So although salience attribution is situated in higher processing stages (Goldstein \& Volkow, 2011), it may well influence earlier visual processing in a top-down fashion. Even more specifically, a study by Murray and Wojciulik (2004) has shown that top-down attention not just increased activity, but also neural selectivity in lateral occipital complex, a region involved in the visual processing of objects and shapes (Murray \& Wojciulik, 2004). Thus one mechanism by which a smoker's attention could be biased towards (salient) visual smoking cues may be via increased processing in the extended visual system.

In accordance with this hypothesis, there is some evidence for increased activation in areas of the primary visual and extra striate cortices in response to smoking cues (Due, Huettel, Hall, \& Rubin, 2002; Engelmann et al., 2012; Havermans, Vuurman, van den Hurk, Hoogsteder, \& van Schayck, 2014; Janes, Pizzagalli, Richardt, Frederick, Holmes, et al., 2010). Nevertheless, the evidence remains scarce, and existing theories on attention bias have explicitly argued for a role of the brain's reward pathway and not the extended visual system in this process (Everitt \& Robbins, 2005; Robinson \& Berridge, 2008). A reason for the inconclusive neuroimaging evidence for the involvement of the visual system, could be that visual processing of salient smoking cues is only slightly enhanced compared to neutral cues, since no motivational value has been attributed in this stage of processing. Standard univariate functional magnetic resonance imaging (fMRI) analysis techniques might not be sufficiently sensitive to detect such subtle effects. Furthermore, in several of these cuereactivity studies the participants had been smoking before scanning (Engelmann et al., 2012; Janes, Pizzagalli, Richardt, Frederick, Holmes, et al., 2010). Since smoking cues are 
of greater salience and relevance for deprived smokers than for satiated smokers (Robinson $\&$ Berridge, 2008), this may have minimized the saliency -and thereby the enhanced visual processing- of the smoking cues.

In the current study, we use functional MRI and a linear classification algorithm (support vector machine (SVM) (Cortes \& Vapnik, 1995) to investigate whether smokingrelated visual cues are processed differently when a smoker is deprived of nicotine compared to a salient state. We investigated the discriminability of neural responses to cigarette images and visually comparable pencil images immediately after the participant has smoked a cigarette, and compare this to the discriminability of the responses when the smoker is craving for nicotine. We hypothesize that the neural responses will grow more distinct for the smoking related cues in the latter situation, as these stimuli will be increasingly behaviourally relevant. This in turn would imply that nicotine deprivation in smokers directly biases early visual object recognition towards smoking-related cues. 


\section{Methods}

\section{Participants}

Fourteen right-handed, currently smoking individuals (mean age 25.21 years, 6 males) participated in this study. All participants reported smoking approximately 10 cigarettes a day for at least one year. Exclusion criteria were history of physical or mental illness, use of psychotropic medication, history of drug or alcohol abuse, and contraindications for the MRI scanner. Participants received financial compensation for their participation and travel costs. The study was approved by the local ethics committee and written informed consent was obtained from each participant.

\section{Procedure}

Participants were scanned on two occasions. On one occasion they were instructed to refrain from smoking for at least 10 hours before the experiment. On the other occasion participants were allowed to smoke ad libitum and were specifically asked to smoke a cigarette just before entering the lab in order to achieve maximal satiation. The order of the sessions was randomized across subjects. Upon entering the lab, smoking status was verified by exhaled carbon monoxide measurement. In addition, participants reported at what time they had smoked their most recent cigarette. Furthermore, they filled out the Fagerström test for nicotine dependency (FTND) (Heatherton, Kozlowski, Frecker, \& Fagerström, 1991), the Minnesota Nicotine Withdrawal Scale (MNWS) (Cappelleri et al., 2005) and an MRI eligibility check.

\section{Localizer run}

An independent functional localizer run preceded the experiment to localize object sensitive regions of the brain. Participants were presented with 12 blocks of 10 grayscale pictures of common objects and 12 blocks of scrambled versions of the same pictures, all equalized for luminance. All pictures were presented for $1200 \mathrm{~ms}$ with an inter-stimulus interval of 600 ms. Stimulus blocks were presented in random order and interleaved with fixation blocks of $12600 \mathrm{~ms}$.

\section{Experimental run}

Stimuli for this run consisted of coloured pictures of cigarettes, pencils and chairs in similar colours, presented centrally on a white background. Participants were presented with three runs, consisting of 8 blocks of 8 pictures of either cigarettes or pencils or chairs. Pictures were presented for $1500 \mathrm{~ms}$ with a $50 \mathrm{~ms}$ inter-stimulus interval. Stimulus blocks were presented in random order and interchanged with intervals of 10, 12 or 14 seconds of rest (see figure 1). These intervals allowed the BOLD response elicited by the previous stimulus 
block to return back to baseline before the onset of the adjacent block. To ensure attention to the pictures, participants had to respond to catch trials in which presentation of the preceding picture was repeated.

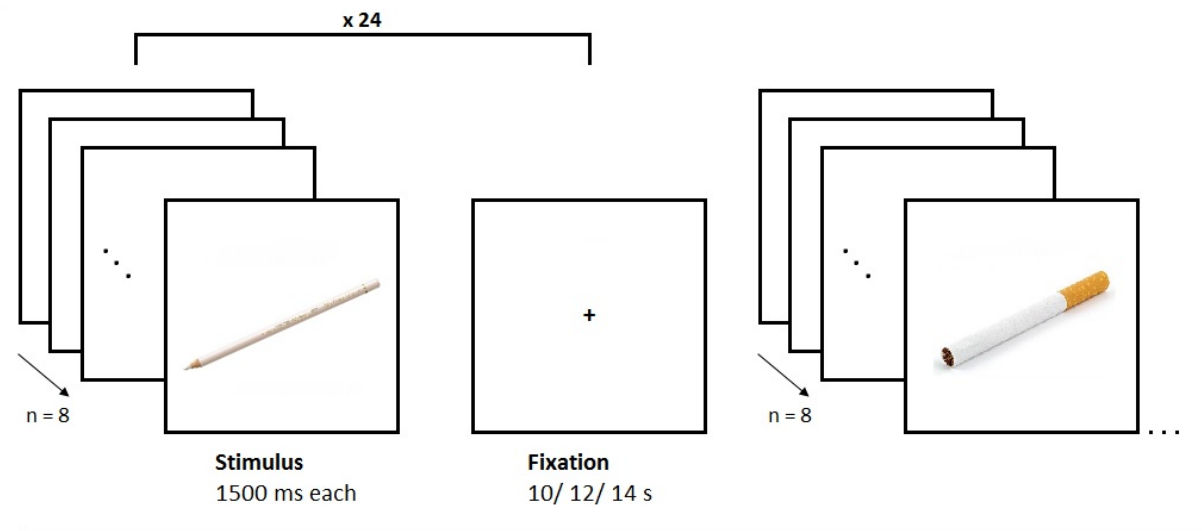

Figure 1. Experimental task paradigm.

\section{Imaging data acquisition}

Functional and anatomical images were acquired in a 3 Tesla Siemens Prisma scanner (Siemens medical systems, Germany) using a 64-channel head coil. For the localizer run, functional images were obtained for 33 contiguous axial slices (voxelsize $=3 \times 3 \times 3 \mathrm{~mm}$ ) using a standard gradient echo planar imaging (EPI) sequence (repetition time [TR] = $1800 \mathrm{~ms}$, echo time $[\mathrm{TE}]=30 \mathrm{~ms}$, matrix size $=72 \times 72$, flip angle $[\mathrm{FA}]=9^{\circ}$ ). Functional images for the experimental runs were also acquired with an EPI sequence (TR $=2000 \mathrm{~ms}$, $\mathrm{TE}=30 \mathrm{~ms}$, matrix size $=72 \times 72, \mathrm{FA}=77^{\circ}$ ), but for 36 contiguous axial slices (voxelsize $3 \times 3 \times 3 \mathrm{~mm}$ ). Both functional measurements covered as much as possible of the entire cortical volume. Whole brain structural images were acquired using a $1 \times 1 \times 1 \mathrm{~mm}$ resolution T1 weighted MPRAGE sequence $\left([\mathrm{TR}]=2250 \mathrm{~ms},[\mathrm{TE}]=2.21 \mathrm{~ms},[\mathrm{FA}]=9^{\circ}\right)$. The first two volumes of all functional runs were discarded because of possible T1 saturation.

Participants were placed in supine position on the scanner bed and their heads were fixated with foam pads. Stimuli were projected on a screen at the back of the scanner bore, and were visible to the participants through a mirror attached to the head coil. Responses were made with a hand-held button box. Stimulus presentation was accomplished with Presentation software (Neurobehavioral Systems Inc., Albany, CA) for the localizer run and 
E-prime® (Psychology Software Tools, Inc. Sharpsburg, PA) for the experimental runs and was synchronised to the MR data acquisition.

\section{Analysis}

Imaging data was pre-processed and analyzed using Brainvoyager QX version 2.8 (Brain Innovation, Maastricht, The Netherlands). First, functional images were corrected for possible susceptibility-induced EPI distortions by using B0 field maps and the Brainvoyager QX plugin Anatabacus (Breman, Peters, Weiskopf, Ashburner, \& Goebel, 2009). The functional data were then corrected for slice scan-time differences and 3D head motion (6 parameters). In order to enhance the subsequent alignment of the functional images to the anatomical volume, the first and third runs were corrected with the second run as intra-session reference, as the acquisition of this run was temporally adjacent to the anatomical scan. Subsequently, linear trends and low frequency temporal drifts were removed from the data using a high-pass filter. The resulting functional data were coregistered to the anatomical volume and transformed to Talairach space. To further reduce the effects of motion-related variability ('spikes'), a custom Matlab R2014b (Mathworks Inc., Natick, MA) algorithm was applied that for each volume computed the percentage of voxels that exceeded the voxel mean intensity by more than 4 standard deviations. Volumes with more than $2 \%$ extreme voxels were labelled as affected by 'excessive' motion. These volumes were replaced by new values derived from a voxel-wise spline interpolation between the volumes that temporally bordered the removed volumes. In case the excessive volumes were at the beginning or end of the run, the volumes were simply removed.

A standard whole brain univariate random effects GLM analysis with subject as random factor was performed to localize bilateral LOC clusters across subjects. The locations of the object selective regions-of-interest (ROI's) were determined based on a conjunction contrast and anatomical criteria. We selected voxels within bilateral ventral occipitotemporal cortex that showed a significant response (Bonferroni corrected) in the conjunction analysis between an object responsive contrast (objects > scrambled objects) and an object positive contrast (objects > baseline).

The experimental runs were analysed through a pattern classification approach using custom written code in MATLAB R2014a (Mathworks Inc., Natick, MA). This analysis was confined to the resulting voxels from the analysis of the localizer run. First, for each voxel within the region-of-interest, individual responses to the experimental trials were estimated by fitting a double-gamma hemodynamic response function (HRF) to the voxel's time course, using the resulting beta as trial estimate. For each ROI, this resulted in a matrix with dimensions $\mathrm{VxT}$, where $\mathrm{V}$ represents the number of voxels, and $\mathrm{T}$ stands for the number of trials. The trials were labeled according to their corresponding condition 
(cigarette, pencil, chair), and normalized to a mean of 0 and a standard deviation of 1 . The analysis was run using a leave-1-run-out cross-validation. First for the cigarette and pencil conditions, the dataset was split into a training set consisting of two runs (i.e. 16 examples) per condition and a test set consisting of one run (i.e. 8 examples) per condition. The labelled training trials were submitted to a linear support vector machine classifier (Mourão-Miranda, Bokde, Born, Hampel, \& Stetter, 2005), which performs binary classification on a dataset by placing all cases in a multidimensional space. Each individual case (or example) is expressed as a vector of $\mathrm{N}$ features in the $\mathrm{N}$-dimensional space. The algorithm then defines an optimal separation boundary, or hyperplane, between the two classes, given the training data. The generalizability of the trained classifier was subsequently assessed by feeding the independent and unlabelled test trials to the algorithm. The accuracy at which the classifier is able to determine the correct labels from these trials given only the response patterns is an indication of successful learning of the algorithm. This, in turn, reflects a meaningful difference in spatial patterns of neural activity elicited by the two conditions.

These steps were repeated three times with different runs for training and testing, after which the average prediction accuracy was computed. In addition, we empirically estimated the distribution of prediction accuracies under the null hypothesis by a bootstrapping approach: instead of training the classifier on voxel patterns and corresponding class labels, we pseudo randomly assigned the labels to the patterns (randomization of labels per run). The classifier then learned the arbitrary relationship between patterns and classes. By feeding the test trials to the model we obtained a prediction accuracy under the null hypothesis. Repeating this 1000 times yields the distribution of prediction accuracies under the null hypothesis. This entire analysis was repeated for the cigarette and chair, and pencil and chair classes. Finally, a nonparametric Wilcoxon signed-rank test (Wilcoxon, 1945) was performed to statistically test the mean prediction accuracies against the permutations, and to test the difference between the deprivation and satiation sessions. Resulting $p$-values were corrected for multiple comparisons by computing the false discovery rate (FDR) using $\mathrm{q}=0.05$. 


\section{Results}

\section{Participant characteristics}

Data of four participants had to be discarded because they did not fully complete all experimental runs. The mean Fagerström score of our participants was $2.8(\mathrm{SD}=1.79)$, which reflects a low (to moderate) level of smoking dependence (Heatherton et al., 1991). On average, participants were smoking approximately 10 cigarettes a day, and there was no difference in the amount of cigarettes smoked in the weeks preceding each session (mean deprived $=72.7 \mathrm{SD}=36.73$; mean satiated $=76.1, \mathrm{SD}=35.22 ; \mathrm{t}_{(18)}=-.211, \mathrm{p}=.835$ ). Exhaled carbon monoxide values were significantly lower when participants had been deprived of smoking (range deprived: $0-9$, mean $=4.18$; range satiated: $5-13$, mean $=8.00$; $\left.\mathrm{t}_{(18)}=-3.425, \mathrm{p}<.004\right)$. Scores on the MNWS did not differ between the two sessions (mean deprived $=10.90, \mathrm{SD}=5.80$; mean satiated $=10.40, \mathrm{SD}=6.92 ; \mathrm{t}_{(18)}=.175, \mathrm{p}=.863$ ). Moreover, participants did not experience more craving after smoking deprivation (as indicated on a $0-4$ scale; mean deprived $=2.20, \mathrm{SD}=0.92$; mean satiated $=1.70, \mathrm{SD}=$ $\left.1.25 ; \mathrm{t}_{(18)}=1.018, \mathrm{p}=.322\right)$.

\section{Localizer}

Significant bilateral LOC clusters were identified in all but one participants. The average location of these clusters over all participants is shown in figure 2. For one participant the specified contrast yielded no significant voxels with and without bonferroni correction. Therefore the average location of left and right LOC of all other participants was used in the MVPA analysis for this participant. The classification analysis was performed on both left and right LOC combined, as well as on separate left- and right LOC voxels.
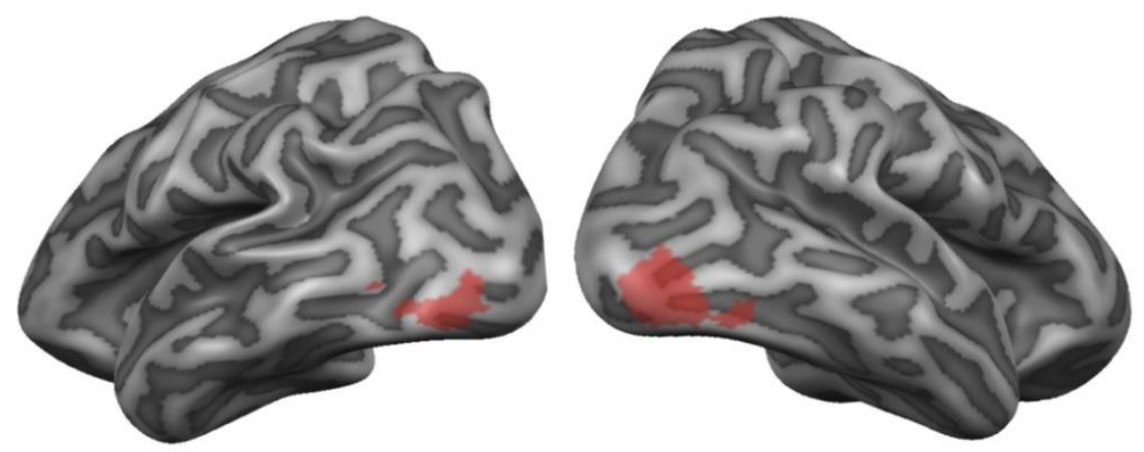

Figure 2. Average LOC clusters. Clusters of object-sensitive voxels within bilateral ventral occipitotemporal cortex, averaged over all participants. Projected on a partially inflated individual cortex reconstruction. 


\section{Multivoxel pattern analysis}

Bilateral LOC

Over the voxels of left and right LOC combined, the classification algorithm was able to discriminate between patterns of activity elicited by visually similar smoking related (cigarettes) and neutral objects (pencils) above empirically estimated chance levels only when participants were smoking deprived (mean $=61.5 \%$, mean permuted $=49.9 \%, \mathrm{p}=$ .009 ) but not when they were satiated (mean $=52.7 \%$, mean permuted $=49.9 \%$, ns.), see figure 2. Moreover, the difference in prediction accuracies between the satiated and deprived conditions was significantly higher than the difference between the permuted accuracies of both conditions (accuracies D-S $=8.75$, permutations $\mathrm{D}-\mathrm{S}=-0.04, \mathrm{p}=.014$ ). Discrimination between the visually less similar smoking related (cigarettes) and neutral (chairs) cues was possible above permuted chance levels when participants were deprived (mean $=74.4 \%$, mean permuted $=49.8 \%, \mathrm{p}=.002)$ and when they were satiated (mean $=$ $72.3 \%$, mean permuted $=50.0 \%, \mathrm{p}=.004)$, and did not differ between conditions (accuracies D-S $=2.08$, permutations $\mathrm{D}-\mathrm{S}=-0.16$, ns.). Similarly, discrimination between two visually distinct neutral objects (pencils, chairs) was possible above empirical chance levels for deprivation (mean $=78.1 \%$, mean permuted $=50.4 \%, \mathrm{p}=.002$ ) and satiety (mean $=74.2 \%$, mean permuted $=49.6 \%, \mathrm{p}=.004)$ and also did not differ between the two conditions (accuracies D-S $=3.96$, permutations $\mathrm{D}-\mathrm{S}=-0.87$, ns.). 


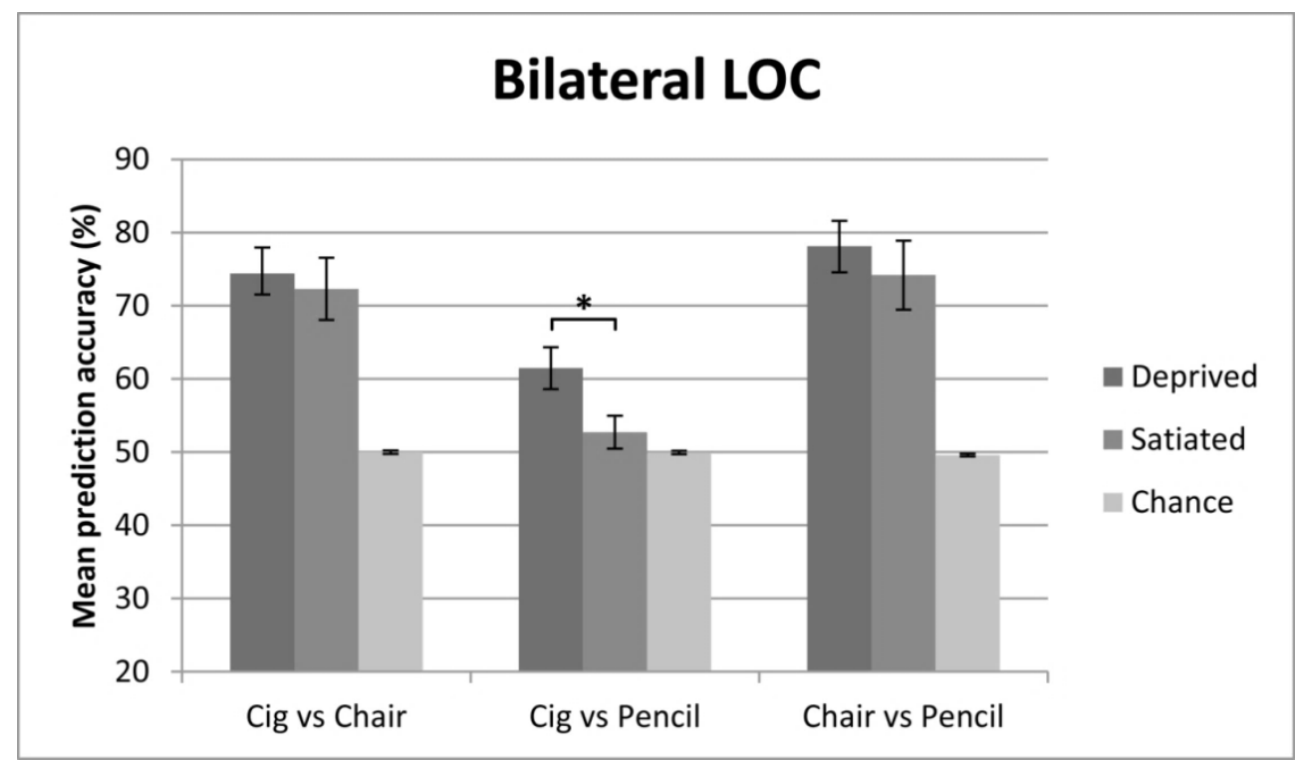

Figure 3. Classification results of all contrasts over bilateral LOC. Prediction accuracies for the cigarettes vs pencils contrast were significantly higher when participants were deprived of smoking $(\mathrm{p}<.02)$. No differences between the deprived and satiated conditions were found for the other condition pairs.

\section{Left and right LOC}

In left LOC, discrimination between response patterns elicited in response to visually similar smoking related (cigarettes) and neutral objects (pencils) was not possible above empirically estimated chance levels when participants were deprived of smoking (mean = $56.7 \%$, mean permuted $=49.8 \%$, ns.) or when they were satiated (mean $=50.6 \%$, mean permuted $=50.9 \%$, ns.). However, the difference in decoding between the deprived and satiated conditions was significantly higher than the difference in empirically estimated chance levels (accuracies D-S $=6.04$, permutations $\mathrm{D}-\mathrm{S}=-1.00, \mathrm{p}=.03$ ). Moreover, the classifier was able to discriminate between responses elicited by the visually less similar smoking related (cigarettes) and neutral (chairs) cues above permuted chance levels in the deprived (mean $=75.6 \%$, mean permuted $=50.5 \%, \mathrm{p}=.002$ ) as well as the satiated (mean $=$ $72.3 \%$, mean permuted $=49.9 \%, p=.004$ ) conditions. In addition, the classifiers ability to discriminate between responses to cigarettes and chairs did not differ between deprivation and satiety (accuracies $\mathrm{D}-\mathrm{S}=3.33$, permutations $\mathrm{D}-\mathrm{S}=0.58$, ns.). Finally discrimination between two neutral objects based on responses in left LOC was possible when participants 
were satiated (mean $72.5 \%$, mean permuted $=50.3 \%, \mathrm{p}=.004$ ) and when they were deprived (mean $=75.6 \%$, mean permuted $=50.5 \%, \mathrm{p}=.002$ ).

In right LOC also, decoding accuracies for the cigarettes - pencils contrast were significantly higher than empirically estimated chance levels in both the satiated (mean = $57.5 \%$, mean permuted $=50.1 \%, \mathrm{p}=.02$ ) and deprived condition (mean $=61.9 \%$, mean permuted $=49.8 \%, \mathrm{p}=.002)$. Moreover, they did not significantly differ between the conditions (accuracies D-S $=4.38$, permutations D-S $=-0.34$, ns.). Similarly, discrimination between cigarettes and chairs was possible above permuted chance levels in satiated (mean $=68.5 \%$, mean permutes $=50.0 \%, \mathrm{p}=.004)$ and deprived (mean $=66.3 \%$, mean permuted $=49.4 \%, \mathrm{p}=.006$ ) participants, and did not differ between conditions (accuracies D-S = 2.29 , permutations $\mathrm{D}-\mathrm{S}=.63$, ns.). Furthermore the classifier was able to discriminate between responses in right LOC to two different neutral objects (pencils and chairs) under conditions of smoking deprivation (mean $=74 \%$, mean permuted $=49.4 \%, \mathrm{p}=.002$ ) and satiety (mean $=68.5 \%$, mean permuted $=50.2 \%, \mathrm{p}=.01$ ). Classification results of left and right LOC are depicted in figure 4. 

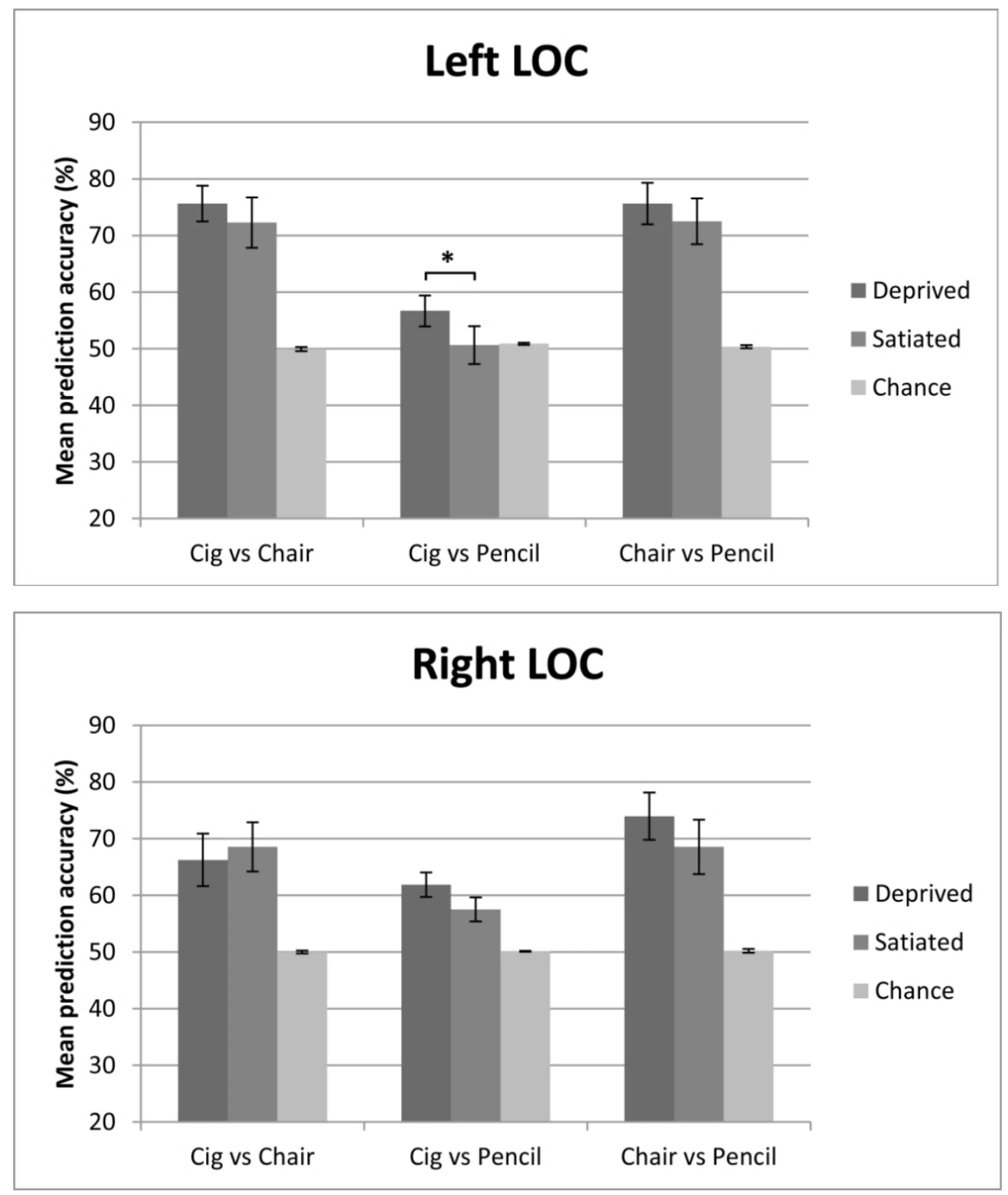

Figure 4. Classification results of all contrasts in left and right LOC separately. In left LOC, decoding accuracies for the cigarettes - pencils contrast were significantly higher in the deprived than in the satiated condition. For all other stimuli contrasts there was no difference in discriminability between the deprived and satiated conditions, in both left and right LOC. 


\section{Discussion}

This study investigated to what extent nicotine deprivation affects early visual processing of smoking-related objects. More specifically, we examined whether the neural representations of images related to smoking become more apparent when smokers are in a state of abstinence. By means of multi-voxel pattern analysis we demonstrated 1) that we are able to discriminate between the visually similar categories cigarettes and pencils above chance level by observing the underlying patterns of activity in object-sensitive LOC, and 2) that this discriminability ceases (particularly in the left hemisphere) when subjects are satiated. From this, we conclude that the effect of abstinence on behaviour can at least be traced back to a basic level of visual object processing. This indicates that the mechanism by which a smoker's attention is biased towards smoking cues potentially affects processing in the early visual system, and is not confined to only higher-order motivation and reward related areas as is generally emphasized in the literature (Hester \& Luijten, 2013; Jasinska, Stein, Kaiser, Naumer, \& Yalachkov, 2014; Volkow, Wang, Tomasi, \& Baler, 2013). In light of the increased salience to smoking related cues after nicotine deprivation (Robinson \& Berridge, 2008), our findings offer a new insight in the possible neural mechanisms that facilitate this behavioural effect.

When our analysis was split between left and right LOC, the specific effect of nicotine deprivation on the processing of smoking related cues was only significant in left LOC. However, without a direct statistical comparison between left and right LOC we cannot draw a definite conclusion about the lateralization of the effect. Nevertheless, we speculate that the apparent lateralization could be due to the involvement of motivational processes that are lateralized to the right hemisphere. Early electrophysiological studies have shown left hemispheric activation in response to appetitive cues. Specifically, approach motivation has been associated with relative left frontal activation, and this lateralization was found to increase in deprived participants (Zinser, Fiore, Davidson, \& Baker, 1999). In our study, lateralized activation of left frontal motivational networks in response to salient (and possibly appetitive) smoking cues may thus have influenced processing in left LOC in deprived participants.

The fact that we only found effects of deprivation on processing for the cigarette pencil contrast is possibly caused by their visually similar appearances. That is, the other smoking vs neutral stimulus contrast consisted of cigarettes and chairs, which have much more distinct visual features. As LOC is specifically sensitive to object shapes (GrillSpector, Kourtzi, \& Kanwisher, 2001; Kim, Biederman, Lescroart, \& Hayworth, 2009), it is plausible that the large difference in shapes between cigarettes and chairs had caused a ceiling effect, due to which differences between the deprived and satiated conditions could not be demonstrated. The large dissimilarity in shape is also very likely to be the reason for 
the much higher prediction accuracies in the cigarette - chair, as well as the pencil - chair conditions, compared to the cigarette - pencil condition.

A few considerations should be taken into account with regard to the design of our study. First, our participants exhibited only low (to moderate) levels of nicotine dependency, as indicated by their low FTND scores. Therefore it is possible that the smoking cues were not as attractive or attention grabbing for them as they would have been for highly dependent individuals. Hence for more dependent smokers, patterns of activity related to smoking cues may have been better discriminable from those related to neutral cues. Moreover, highly dependent smokers would have been more affected by the nicotine deprivation condition, possibly increasing discriminability between smoking and neutral cues in the deprived condition even more. Secondly, it is striking that our deprivation condition was not successful in inducing craving. This could be due to a too short -and overnight- abstinence period, during which participants only missed a few cigarettes. For instance, 16 hours of abstinence have been shown to reliably induce craving (Jarvik et al., 2000), while our participants were only abstinent for only 12 hours on average. Although this difference is small, longer periods of abstinence (in heavier smokers) may lead to more distinct responses to smoking related and neutral cues in LOC. Third, to control as much as possible for other influences, we have kept our images of smoking related and neutral objects very 'clean'. They consisted of just one object in a neutral colour presented centrally on a white background. This may have made our smoking images less attractive and interesting to look at than more lifelike images of smoking scenes in which people enjoy smoking cigarettes. Moreover, the fact that our images did not reflect actual smoking scenes may have compromised the ecologic validity of the study.

Nevertheless we have established that neural response patterns in left LOC differ for smoking related and neutral pictures when participants were deprived of nicotine. This indicates that the well-known attention bias that has often been reported in smokers likely affects basic visual object processing. This finding may provide a new target for smoking cessation interventions; for instance non-invasive brain stimulation techniques may be used to manipulate brain activity in LOC. Moreover it shows that treatment interventions should not just aim to eliminate craving elicited by smoking cues, but should specifically focus on extinction of automatic responses to these cues. 


\section{References}

Amaral, D. G., Behniea, H., \& Kelly, J. L. (2003). Topographic organization of projections from the amygdala to the visual cortex in the macaque monkey. Neuroscience, 118(4), 1099-1120.

Austin, A. J., \& Duka, T. (2012). Mechanisms of attention to conditioned stimuli predictive of a cigarette outcome. Behavioural Brain Research, 232(1), 183-189.

Benowitz, N. L. (2010). Nicotine addiction. The New England Journal of Medicine, 362, 2295-303.

Bradley, B. P., Mogg, K., Wright, T., \& Field, M. (2003). Attentional bias in drug dependence: vigilance for cigarette-related cues in smokers. Psychology of Addictive Behaviors, 17(1), 66-72.

Breman, H., Peters, J., Weiskopf, N., Ashburner, J., \& Goebel, R. (2009). Fast fieldmapbased EPI distortion correction with anatabacus: a plugin for BrainVoyager. In NeuroImage (Vol. 47, p. S81).

Brody, A. L. (2006). Functional brain imaging of tobacco use and dependence. Journal of Psychiatric Research, 40(5), 404-18.

Brody, A. L., Mandelkern, M. A., Olmstead, R. E., Jou, J., Tiongson, E., Allen, V., ... Cohen, M. S. (2007). Neural Substrates of Resisting Craving During Cigarette Cue Exposure. Biological Psychiatry, 62, 642-651.

Cappelleri, J. C., Bushmakin, A. G., Baker, C. L., Merikle, E., Olufade, A. O., \& Gilbert, D. G. (2005). Revealing the multidimensional framework of the Minnesota nicotine withdrawal scale. Current Medical Research and Opinion, 21(5), 749-60.

Claus, E. D., Blaine, S. K., Filbey, F. M., Mayer, A. R., \& Hutchison, K. E. (2013). Association Between Nicotine Dependence Severity, BOLD Response to Smoking Cues, and Functional Connectivity. Neuropsychopharmacology, 38(January), 23632372 .

Cortes, C., \& Vapnik, V. (1995). Support-Vector Networks. Machine Learning, 20(3), 273297.

David, S. P., Munafò, M. R., Johansen-Berg, H., Smith, S. M., Rogers, R. D., Matthews, P. M., \& Walton, R. T. (2005). Ventral striatum/nucleus accumbens activation to smoking-related pictorial cues in smokers and nonsmokers: a functional magnetic 
resonance imaging study. Biological Psychiatry, 58(6), 488-94.

Due, D. L., Huettel, S. A., Hall, W. G., \& Rubin, D. C. (2002). Activation in mesolimbic and visuospatial neural circuits elicited by smoking cues: evidence from functional magnetic resonance imaging. The American Journal of Psychiatry, 159(6), 954-60.

Engelmann, J. M., Versace, F., Robinson, J. D., Minnix, J. a, Lam, C. Y., Cui, Y., ... Cinciripini, P. M. (2012). Neural substrates of smoking cue reactivity: a metaanalysis of fMRI studies. NeuroImage, 60(1), 252-62.

Everitt, B. J., \& Robbins, T. W. (2005). Neural systems of reinforcement for drug addiction: from actions to habits to compulsion. Nature Neuroscience, 8(11), 14811489.

Field, M., \& Cox, W. M. (2008). Attentional bias in addictive behaviors: a review of its development, causes, and consequences. Drug and Alcohol Dependence, 97(1-2), 120.

Field, M., Mogg, K., \& Bradley, B. P. (2004). Eye movements to smoking-related cues: effects of nicotine deprivation. Psychopharmacology, 173(1-2), 116-23.

Franklin, T. R., Wang, Z., Wang, J., Sciortino, N., Harper, D., Li, Y., ... Childress, A. R. (2007). Limbic activation to cigarette smoking cues independent of nicotine withdrawal: a perfusion fMRI study. Neuropsychopharmacology, 32(11), 2301-2309.

Goldstein, R. Z., \& Volkow, N. D. (2011). Dysfunction of the prefrontal cortex in addiction: neuroimaging findings and clinical implications. Nature Reviews. Neuroscience, 12(11), 652-69.

Grill-Spector, K., Kourtzi, Z., \& Kanwisher, N. (2001). The lateral occipital complex and its role in object recognition. Vision Research, 41(10-11), 1409-1422.

Gross, T. M., Jarvik, M. E., \& Rosenblatt, M. R. (1993). Nicotine abstinence produces content-specific stroop interference. Psychopharmacology, 110(3), 333-336.

Havermans, A., Vuurman, E. F. P. M., van den Hurk, J., Hoogsteder, P., \& van Schayck, O. C. P. (2014). Treatment with a nicotine vaccine does not lead to changes in brain activity during smoking cue exposure or a working memory task. Addiction, 109(8), $1260-7$.

Heatherton, T. F., Kozlowski, L. T., Frecker, R. C., \& Fagerström, K. O. (1991). The Fagerström Test for Nicotine Dependence: a revision of the Fagerström Tolerance Questionnaire. British Journal of Addiction, 86, 1119-1127. 
Hester, R., \& Luijten, M. (2013). Neural correlates of attentional bias in addiction. CNS Spectrums, (August), 1-8.

Hogarth, L. C., Mogg, K., Bradley, B. P., Duka, T., \& Dickinson, A. (2003). Attentional orienting towards smoking-related stimuli. Behavioural Pharmacology, 14, 153-160.

Janes, A. C., Pizzagalli, D. A., Richardt, S., Frederick, B. deB., Chuzi, S., Pachas, G., ... Kaufman, M. J. (2010). Brain reactivity to smoking cues prior to smoking cessation predicts ability to maintain tobacco abstinence. Biological Psychiatry, 67(8), 722729.

Janes, A. C., Pizzagalli, D. A., Richardt, S., Frederick, B. deB., Holmes, A. J., Sousa, J., ... Kaufman, M. J. (2010). Neural substrates of attentional bias for smoking-related cues: an FMRI study. Neuropsychopharmacology, 35(12), 2339-2345.

Jarvik, M. E., Madsen, D. C., Olmstead, R. E., Iwamoto-Schaap, P. N., Elins, J. L., \& Benowitz, N. L. (2000). Nicotine Blood Levels and Subjective Craving for Cigarettes. Pharmacology Biochemistry and Behavior, 66(3), 553-558.

Jasinska, A. J., Stein, E. A., Kaiser, J., Naumer, M. J., \& Yalachkov, Y. (2014). Factors modulating neural reactivity to drug cues in addiction: a survey of human neuroimaging studies. Neuroscience and Biobehavioral Reviews, 38, 1-16.

Kerns, J. G., Cohen, J. D., MacDonald, A. W., Cho, R. Y., Stenger, V. A., \& Carter, C. S. (2004). Anterior cingulate conflict monitoring and adjustments in control. Science, 303(5660), 1023-6.

Kim, J. G., Biederman, I., Lescroart, M. D., \& Hayworth, K. J. (2009). Adaptation to objects in the lateral occipital complex (LOC): Shape or semantics? Vision Research, 49(18), 2297-2305.

Luijten, M., Veltman, D. J., van den Brink, W., Hester, R., Field, M., Smits, M., \& Franken, I. H. A. (2011). Neurobiological substrate of smoking-related attentional bias. NeuroImage, 54(3), 2374-81.

Mogg, K., \& Bradley, B. P. (2002). Selective processing of smoking-related cues in smokers: manipulation of deprivation level and comparison of three measures of processing bias. Journal of Psychopharmacology, 16(4), 385-392.

Mourão-Miranda, J., Bokde, A. L. W., Born, C., Hampel, H., \& Stetter, M. (2005). Classifying brain states and determining the discriminating activation patterns: Support Vector Machine on functional MRI data. NeuroImage, 28(4), 980-95. 
Murray, S. O., \& Wojciulik, E. (2004). Attention increases neural selectivity in the human lateral occipital complex. Nature Neuroscience, 7(1), 70-4.

Phillips, M. L., Drevets, W. C., Rauch, S. L., \& Lane, R. (2003). Neurobiology of emotion perception I: The neural basis of normal emotion perception. Biological Psychiatry, $54,504-514$.

Robinson, T. E., \& Berridge, K. C. (2008). The incentive sensitization theory of addiction: some current issues. Philosophical Transactions of the Royal Society of London. Series B, Biological Sciences, 363(July), 3137-3146.

Rubinstein, M. L., Luks, T. L., Moscicki, A. B., Dryden, W., Rait, M. ., \& Simpson, G. V. (2010). Smoking-related cue-induced brain activation in adolescent light smokers. Journal of Adolescent Health, 48(1), 7-12.

Sayette, M. A., \& Hufford, M. R. (1994). Effects of cue exposure and deprivation on cognitive resources in smokers. Journal of Abnormal Psychology, 103(4), 812-818.

Tanaka, K. (1992). Inferotemporal cortex and higher visual functions. Current Opinion in Neurobiology, 2, 502-505.

Volkow, N. D., \& Fowler, J. S. (2000). Addiction, a disease of compulsion and drive: involvement of the orbitofrontal cortex. Cerebral Cortex, 10, 318-325.

Volkow, N. D., Wang, G. J., Tomasi, D., \& Baler, R. D. (2013). Unbalanced neuronal circuits in addiction. Current Opinion in Neurobiology, 23(4), 639-648.

Watanabe, M., Hikosaka, K., Sakagami, M., \& Shirakawa, S. (2002). Coding and Monitoring of Motivational Context in the Primate Prefrontal Cortex. Journal of Neuroscience, 22(6), 2391-2400.

Waters, A. J., \& Feyerabend, C. (2000). Determinants and effects of attentional bias in smokers. Psychology of Addictive Behaviors, 14(2), 111-120.

Wilcoxon, F. (1945). Individual comparisons by ranking methods. Biometrics Bulletin, 1(6), 80-83.

Wilson, S. J., Sayette, M. A., \& Fiez, J. A. (2004). Prefrontal responses to drug cues: a neurocognitive analysis. Nature Neuroscience, 7(3), 211-4.

Zack, M., Belsito, L., Scher, R., Eissenberg, T., \& Corrigall, W. A. (2001). Effects of abstinence and smoking on information processing in adolescent smokers. Psychopharmacology, 153, 249-257. 
Zinser, M. C., Fiore, M. C., Davidson, R. J., \& Baker, T. B. (1999). Manipulation smoking motivation: Impact on an electrophysiological index of approach motivation. Journal of Abnormal Psychology, 108(2), 240-254. 
Neural and cognitive determinants of smoking addiction and cessation 


\section{CHAPTER 3}

Neural representation of smoking-related cues under influence of smoking deprivation: a multivariate searchlight approach.

Havermans, A., van Schayck C.P., Vuurman, E.F.P.M., Riedel, W.J., \& van den Hurk, J. Neural representation of smoking-related cues under influence of smoking deprivation: a multivariate searchlight approach. (in preparation). 


\begin{abstract}
Smokers are characterized by enhanced selective attention for smoking related cues. In this study we used a multivariate searchlight approach to identify brain regions involved in the biased processing of these cues. Fourteen current smokers ( $\geq 10$ cigarettes a day) were scanned in two sessions: once after 10 hours of nicotine abstinence and once after smoking ad libitum. Regional blood oxygenated level dependent (BOLD) response was measured while participants were presented with 24 blocks of 8 colour-matched pictures of cigarettes, pencils or chairs. The functional data were analysed through a pattern classification approach. The multivariate searchlight identified bilateral lateral occipital cortex regions showing better decoding of neural response patterns to visually similar smoking related (cigarettes) and neutral (pencils) images when participants were deprived of smoking. Moreover, the left precentral gyrus showed discriminable responses to smoking related and neutral cues during satiety. These findings support the results of our previous study, indicating that the mechanism by which a smoker's attention is biased towards smoking cues indeed affects processing in the early visual system.
\end{abstract}




\section{Introduction}

Conditioned responses to smoking cues play a major role in the maintenance of smoking addiction. When specific cues in the environment are paired with the rewarding effects of nicotine, smokers will start to learn this association through a process of conditioning. As a consequence, these cues will signal the possibility of future reward and induce a state of 'wanting' by activating the mesolimbic system (Hester \& Luijten, 2013). Over time, smoking related cues can become extremely salient and trigger craving for cigarettes (Benowitz, 2010; Robinson \& Berridge, 2008). Moreover, smokers will automatically focus and retain their attention on these cues, a process known as attention bias (Field \& Cox, 2008). Cue-elicited craving and attention bias are closely associated and have both been implicated in the maintenance of smoking addiction (Field \& Cox, 2008; Field, Marhe, \& Franken, 2014; Franken, 2003). That is, biased attention will lead to increased detection of, and exposure to, craving eliciting smoking related cues (Conklin et al., 2015). Whereas smoking cues are thought to become more salient when smokers experience craving, thereby increasingly attracting smokers' attention (Robinson \& Berridge, 2008). In sum, episodes of craving triggered by attended smoking related cues may impair smokers' attempts to remain abstinent and cause relapses in those who do quit (eg. Janes, Pizzagalli, Richardt, Frederick, Chuzi, et al., 2010; Waters et al., 2003). Disrupting automatic conditioned responses to smoking cues may thus facilitate smoking cessation and prevent relapses.

In order to find specific targets for intervention it is important to understand the underlying neural mechanisms of these processes. Various neuroimaging studies have investigated brain responses to smoking related cues and have indicated the involvement of several neural networks. First of all, activation in response to smoking related cues has been found in areas of the limbic system, such as ventral striatum/ nucleus accumbens (David et al., 2005; Franklin et al., 2007; Nestor, McCabe, Jones, Clancy, \& Garavan, 2011) and amygdala (Due, Huettel, Hall, \& Rubin, 2002; Franklin et al., 2007; Janes, Pizzagalli, Richardt, Frederick, Holmes, et al., 2010; Nestor et al., 2011). Activation of these regions in response to smoking cues likely reflects the rewarding value of the cues and the motivational processes guiding drug-seeking behaviour. The amygdala in particular is thought to play a key role in the processing of salient stimuli, as increased amygdala activity may bias processing in favor of such cues. This is accomplished via projections from the amygdala to frontal areas that control the allocation of attentional resources (Pessoa \& Ungerleider, 2004). In line with this, frontal and parietal attentional networks have also been shown to respond to smoking related cues. Specifically, the anterior cingulate cortex (Janes, Pizzagalli, Richardt, Frederick, Holmes, et al., 2010; Luijten et al., 2011; McBride, Barrett, Kelly, Aw, \& Dagher, 2006; McClernon, Hiott, Huettel, \& Rose, 
2005; Nestor et al., 2011), the superior frontal gyrus (McClernon et al., 2005), the superior parietal lobule and the superior temporal gyrus (Janes, Pizzagalli, Richardt, Frederick, Holmes, et al., 2010; Luijten et al., 2011; Nestor et al., 2011) have been implicated amongst others. Moreover, the amygdala also sends feedback projections to various structures in the visual ventral stream (Pessoa \& Ungerleider, 2004). Indeed, there is evidence for increased activation in primary visual cortex and visual association areas in response to smoking cues (Due et al., 2002; Engelmann et al., 2012; Havermans, Vuurman, van den Hurk, Hoogsteder, \& van Schayck, 2014; Janes, Pizzagalli, Richardt, Frederick, Holmes, et al., 2010). This indicates that cue saliency may affect processing as early as at the visual level.

According to the incentive sensitization theory of drug use, the perceived reward value of a drug cue increases during abstinence (Robinson \& Berridge, 2008). Moreover, abstinence induced higher levels of craving may also increase attention bias. Therefore neural changes may take place causing increased responses to smoking cues when smokers are abstinent. However, two studies failed to show such an effect (McBride et al., 2006; McClernon et al., 2005), and one other study found abstinence to decrease reactivity in ventral striatum in female smokers (David et al., 2007). Studies that directly contrasted smoking cue reactivity in abstinent and satiated conditions, demonstrated increased responses during abstinence in prefrontal, parietal, cingulate, occipital, and central cortical areas, as well as the dorsal striatum (caudate) (Janes et al., 2009; McClernon, Kozink, Lutz, \& Rose, 2009). Moreover, one meta-analysis study showed increased activity in superior frontal and lingual gyrus in abstinent compared to satiated smokers (Engelmann et al., 2012).

In sum, there is no definite consensus in the literature on the effects of nicotine abstinence on neural responses to smoking related cues. Moreover, it is still unclear how far in the processing hierarchy differences in processing of smoking related and neutral cues can be traced back. In a recent study, we addressed these issues by using functional magnetic resonance imaging (fMRI) and multi-voxel pattern analysis (MVPA) to investigate subtle differences in basic visual processing of smoking related and neutral objects (chapter 2). MVPA analysis techniques employ the spatial distribution of neural responses, and are thereby able to pick up information that is too subtle to be discovered by traditional univariate analyses (De Martino et al., 2008). We were able to discriminate between the visually similar categories cigarettes and pencils above chance level by observing the underlying patterns of activity in object-sensitive area lateral occipital complex (LOC). Moreover, we found that the ability to discriminate between these stimulus categories ceased (particularly in the left hemisphere) when the participants were satiated. We concluded that the mechanism by which a smoker's attention is biased towards smoking cues does not affect processing in the LOC. Thus, feedback projections from the 
amygdala and projections from frontal and parietal attentional networks may not reach the LOC. However, they may well influence processing in other areas, for instance in higher levels of the ventral visual stream. Therefore the current study aimed to identify regions of the brain that show discriminable neural response patterns to smoking related and neutral cues, under influence of smoking deprivation. We used a multivariate searchlight approach to reveal local patterns of voxels throughout the brain that carry information about the cigarette - pencil contrast, and compared the resulting information maps between conditions of satiety and deprivation. 


\section{Methods}

\section{Participants}

Fourteen right-handed, currently smoking individuals (mean age 25.21 years, 6 males) participated in this study. All participants reported smoking approximately 10 cigarettes a day for at least one year. Exclusion criteria were history of physical or mental illness, use of psychotropic medication, history of drug or alcohol abuse, or contraindications for the MRI scanner. Participants received financial compensation for their participation and travel costs. The study was approved by the local ethics committee and written informed consent was obtained from each participant.

\section{Procedure}

Participants were scanned on two occasions. On one occasion they were instructed to refrain from smoking for at least 10 hours before the experiment. On the other occasion participants were allowed to smoke ad libitum and were specifically asked to smoke a cigarette just before entering the lab in order to achieve maximal satiation. The order of the sessions was randomized. Upon entering the lab, smoking status was verified by exhaled carbon monoxide measurement. In addition, participants reported at what time they had smoked their most recent cigarette. Furthermore, they filled out the Fagerström test for nicotine dependency (FTND) (Heatherton, Kozlowski, Frecker, \& Fagerström, 1991), the Minnesota Nicotine Withdrawal Scale (MNWS) (Cappelleri et al., 2005) and an MRI eligibility check.

\section{Experimental run}

The stimuli, experimental procedure and data acquisition parameters were identical to those used in the previous study by Havermans and colleagues (chapter 2). Stimuli consisted of colour pictures of cigarettes, pencils and chairs in similar colours, presented centrally on a white background. Participants underwent three runs, consisting of 8 blocks of 8 pictures of either cigarettes or pencils or chairs. Pictures were presented for $1500 \mathrm{~ms}$ with a $50 \mathrm{~ms}$ inter-stimulus interval. Stimulus blocks were presented in random order and interchanged with intervals of 10, 12 or 14 seconds of rest. These intervals allowed the BOLD response elicited by the previous stimulus block to return back to baseline before the onset of the adjacent block. To ensure attention to the pictures, participants had to respond to catch trials in which presentation of the preceding picture was repeated. 


\section{Imaging data acquisition}

Functional and anatomical images were acquired in a 3 tesla Siemens Prisma scanner (Siemens medical systems, Germany) using a 64-channel head coil. For the localizer run, functional images were obtained for 33 contiguous axial slices $($ voxelsize $=3 \times 3 \times 3 \mathrm{~mm}$ ) using a standard gradient echo planar imaging (EPI) sequence (repetition time $[\mathrm{TR}]=$ $1800 \mathrm{~ms}$, echo time $[\mathrm{TE}]=30 \mathrm{~ms}$, matrix size $=72 \times 72$, flip angle $\left.[\mathrm{FA}]=9^{\circ}\right)$. Functional images for the experimental runs were also acquired with an EPI sequence $([T R]=2000 \mathrm{~ms}$, $[\mathrm{TE}]=30 \mathrm{~ms}$, matrix size $=72 \times 72,[\mathrm{FA}]=77^{\circ}$ ), but for 36 contiguous axial slices (voxelsize $3 \times 3 \times 3 \mathrm{~mm}$ ). Both functional measurements covered as much as possible of the entire cortical volume. Whole brain structural images were acquired using a 1x1x1 mm resolution $\mathrm{T} 1$ weighted MPRAGE sequence $\left([\mathrm{TR}]=2250 \mathrm{~ms},[\mathrm{TE}]=2.21 \mathrm{~ms},[\mathrm{FA}]=9^{\circ}\right)$. The first two volumes of all functional runs were discarded because of possible T1 saturation.

Participants were placed in supine position on the scanner bed and their heads were fixated with foam pads. Stimuli were projected on a screen at the back of the scanner bore, and were visible to the participants through a mirror attached to the head coil. Responses were made with a hand-held button box. Stimulus presentation was accomplished with Presentation software (Neurobehavioral Systems Inc, Albany, CA) for the localizer run and E-prime ${ }^{\circledR}$ (Psychology Software Tools, Inc. Sharpsburg, PA) for the experimental runs and was synchronised to the MR data acquisition.

\section{Data processing}

Imaging data was pre-processed and analyzed using Brainvoyager QX version 2.8 (Brain Innovation, Maastricht, The Netherlands). First, functional images were corrected for possible susceptibility-induced EPI distortions by using B0 field maps and the Brainvoyager QX plugin Anatabacus (Breman, Peters, Weiskopf, Ashburner, \& Goebel, 2009). The functional data were then corrected for slice scan-time differences and $3 D$ head motion (6 parameters). In order to enhance the subsequent alignment of the functional images to the anatomical volume, the first and third runs were corrected with the second run as intra-session reference, as the acquisition of this run was temporally adjacent to the anatomical scan. Subsequently, linear trends and low frequency temporal drifts were removed from the data using a high-pass filter. The resulting functional data were coregistered to the anatomical volume and transformed to Talairach space. To further reduce the effects of motion-related variability, a custom Matlab R2012b (Mathworks Inc, Natick, MA) algorithm was applied that for each volume computed the percentage of voxels that exceeded the voxel mean intensity by more than 4 standard deviations. Volumes with more than $2 \%$ extreme voxels were labelled as affected by 'excessive' motion. These volumes 
were replaced by new values derived from a voxel-wise spline interpolation between the volumes that temporally bordered the removed volumes. In case the excessive volumes were at the beginning or end of the run, the volumes were simply removed.

\section{Multivariate analyses}

In order to examine differences in multivariate information between the stimulus conditions, we used a multivariate searchlight approach. Unlike a mass-univariate method like the general linear model (GLM), a multivariate searchlight does not treat individual voxels as independent variables. Instead, this descriptive method uses a spherical 'searchlight' that centers on every voxel of the cortical volume and gives an estimate of the presence of information in the spherical surround. On a single subject level, the algorithm targets all voxels individually and selects the voxels within the searchlight sphere with predefined radius surrounding the centered voxel. The voxels within this sphere are submitted to an algorithm that performs binary classification on a dataset by placing all cases in a multidimensional space. Each individual case (or example) is expressed as a vector of $\mathrm{N}$ features in the $\mathrm{N}$-dimensional space. The algorithm then defines an optimal separation boundary, or hyperplane, between the two classes, given the training data. The generalizability of the trained classifier was subsequently assessed by feeding the independent and unlabelled test trials to the algorithm. The accuracy at which the classifier is able to determine the correct labels from these trials given only the response patterns is an indication of successful learning of the algorithm. This, in turn, reflects a meaningful difference in spatial patterns of neural activity elicited by the two conditions. The resulting prediction accuracy is returned to the centre voxel, and this procedure is repeated for all voxels in a volume.

In this experiment, the analysis was performed using custom written code in MATLAB R2014a (Mathworks Inc., Natick, MA). First, for each voxel, individual responses to the experimental trials were estimated by fitting a double-gamma hemodynamic response function (HRF) to the voxel's time course, using the resulting beta as trial estimate. Then, at each voxel, a sphere was defined with a radius of 4 voxels surrounding the center voxel. For each sphere, this resulted in a matrix with dimensions VxT, where V represents the number of voxels within the sphere, and $\mathrm{T}$ stands for the number of trials. The trials were labeled according to their corresponding condition (cigarette, pencil) and submitted to a linear support vector machine classifier (MourãoMiranda, Bokde, Born, Hampel, \& Stetter, 2005), using leave-1-run-out cross-validation. Maps were obtained for the satiated and deprived conditions separately, and tested against chance level. Moreover, for each participant a difference map was created by subtracting the prediction accuracy in the satiated state from that in deprived condition in every voxel 
of the maps, and this difference was tested against 0 using a (nonparametric) Wilcoxon signed rank test. Finally a t-test was run across all subjects' difference maps, to locate brain regions where the deprived/satiated difference was significantly larger than zero. Maps were evaluated with a single voxel significance threshold at $\mathrm{p}<.05$, thresholded with a cluster threshold (cluster size determined by a Monte Carlo simulation (1000 iterations) as implemented in Brainvoyager QX 2.8). 


\section{Results}

\section{Participant characteristics}

Data of four participants had to be discarded because they did not fully complete all experimental runs. The mean Fagerström score of our participants was $2.8(\mathrm{SD}=1.79)$, which reflects a low (to moderate) level of smoking dependence (Heatherton et al., 1991). There was no difference in the amount of cigarettes smoked in the weeks preceding each session (mean deprived $=72.7 \mathrm{SD}=36.73$; mean satiated $=76.1, \mathrm{SD}=35.22 ; \mathrm{t}_{(18)}=-.211$, $\mathrm{p}=.835$ ). Exhaled carbon monoxide values were significantly lower when participants had been deprived of smoking (range deprived: $0-9$, mean $=4.18$; range satiated: $5-13$, mean $=$ $\left.8.00 ; \mathrm{t}_{(18)}=-3.425, \mathrm{p}<.004\right)$. Scores on the MNWS did not differ between the two sessions (mean deprived $=10.90, \mathrm{SD}=5.80 ;$ mean satiated $=10.40, \mathrm{SD}=6.92 ; \mathrm{t}_{(18)}=.175, \mathrm{p}=.863$ ). Moreover, participants did not experience more craving after smoking deprivation (as indicated on a $0-4$ scale; mean deprived $=2.20, \mathrm{SD}=0.92$; mean satiated $=1.70, \mathrm{SD}=$ $\left.1.25 ; \mathrm{t}_{(18)}=1.018, \mathrm{p}=.322\right)$.

\section{Multivariate searchlight analysis}

In the satiated condition several regions showed a significant difference in decoding for smoking related and neutral images, including dorsal prefrontal cortex, central sulcus and occipital areas. When smokers were deprived of smoking, temporal, parietal and also occipital areas elicit significantly distinct patterns of activity in response to smoking related and neutral images. Searchlight maps of the separate sessions are depicted in figure 1. 

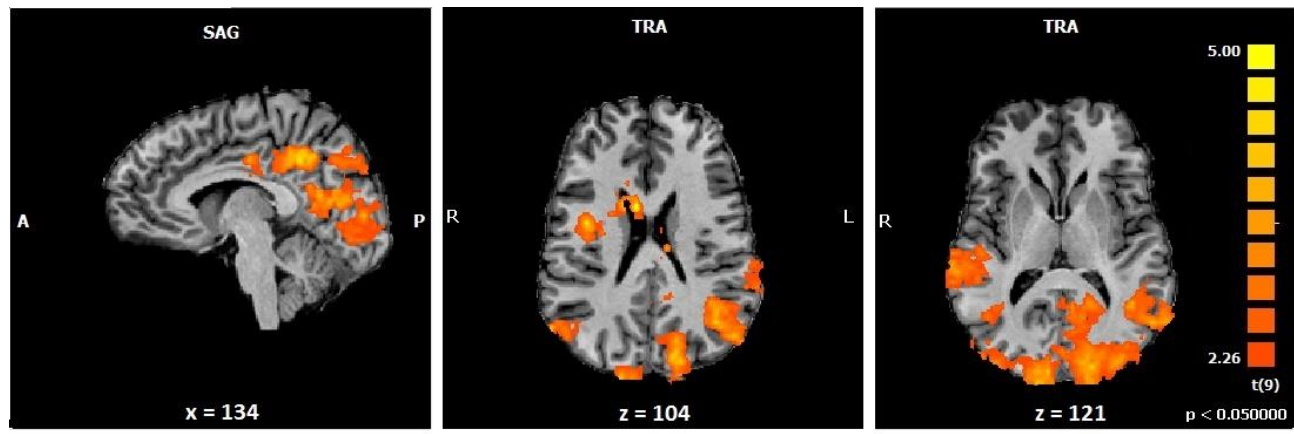

Deprived condition
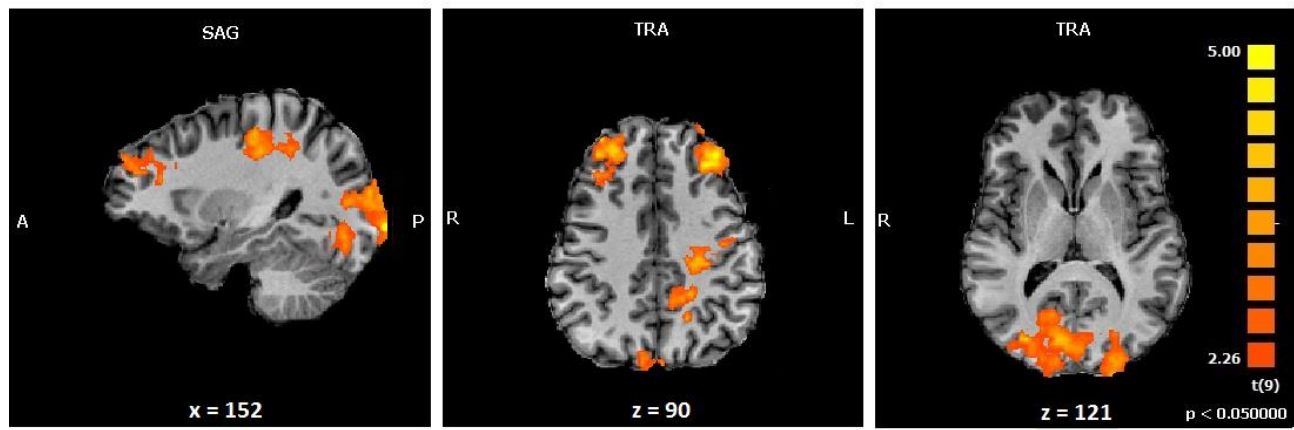

Satiated condition

Figure 1. Searchlight results for deprived and satiated condition. Brain regions that show significantly distinct neural response patterns to smoking related and neutral cues during deprivation (top row) and satiety (bottom row). 


\section{Comparison deprivation and satiety}

Individual difference maps show several brain areas that elicited significantly discriminable neural responses to smoking related and neutral cues, in either the satiety or deprivation condition. A group level t-test over the individual difference maps indicated that the difference in prediction accuracy between the satiated and deprived condition was larger than zero in several brain regions (see figure 2). Specifically, left precentral gyrus shows better decoding when participants were satiated, whereas bilateral lateral occipital regions show significantly better decoding during the deprived condition.
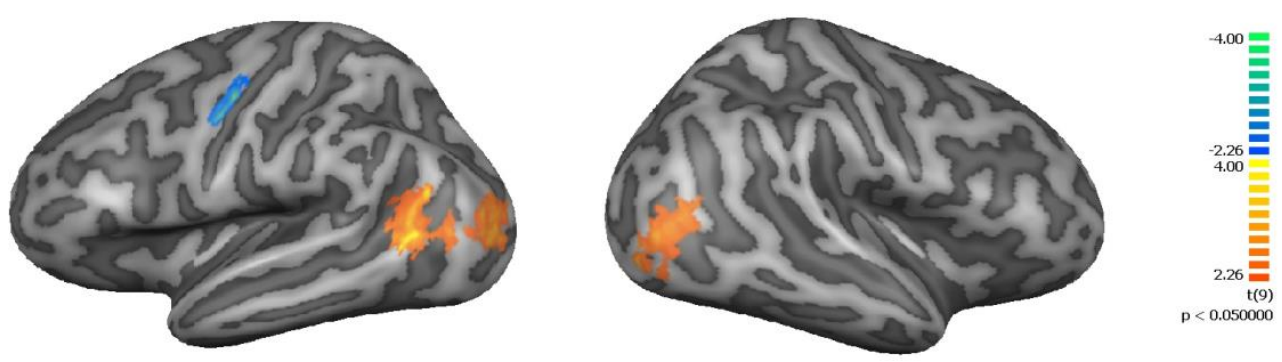

Figure 2. Brain regions that show a significant difference in prediction accuracy between satiated and deprived conditions. Higher prediction accuracies during satiety (indicated by blue colours) are found in left precentral sulcus. For deprived participants, prediction accuracies were higher in bilateral lateral occipital clusters (indicated by orange colours). Projected on an inflated individual cortex reconstruction. 


\section{Discussion}

The aim of this study was to identify brain regions that show discriminable responses to smoking related and neutral cues under influence of smoking abstinence. By means of a multivariate searchlight approach we showed that in the deprived and the satiated condition, various -mainly temporal, parietal and occipital cortical- regions show discriminable neural responses to smoking related and neutral cues. Moreover, when directly comparing neural responses between the two conditions, we found that bilateral lateral occipital cortex regions showed better decoding of neural response patterns to visually similar smoking related (cigarettes) and neutral (pencils) images when participants were deprived of smoking. Furthermore, the left precentral gyrus showed discriminable responses to smoking related and neutral cues only during satiety.

These findings correspond with the results of our previous study in which we demonstrated by means of multivoxel pattern analysis that discriminability between smoking related and non-smoking related visual objects was elevated in object-selective brain region LOC after a period of nicotine abstinence. Our current findings thus support our hypothesis that the mechanism by which a smoker's attention is biased towards smoking cues affects processing in the early visual system. Other areas, such as frontal and parietal attention networks and the amygdala and other motivation related areas have been implicated in this mechanism as well and may be the sources of input for the LOC (Engelmann et al., 2012; Janes, Pizzagalli, Richardt, Frederick, Holmes, et al., 2010; McClernon et al., 2009). Therefore it may be surprising that we did not identify any other areas showing increased discriminability between smoking related and neutral cues in deprived smokers. However, this could simply be due to our small sample size and strict cluster threshold. In order to find out which brain areas influence selective processing of smoking related cues in LOC, it would be interesting for future studies to perform a seedbased connectivity analysis, using LOC as a seed. This way, it may be possible to determine whether processing in LOC is biased by direct feedback projections from the amygdala, or indirectly via attentional networks, or in both ways.

The identified area in the left precentral gyrus seems to correspond to the handregion of the primary motor cortex. Participants were instructed to respond to specific catch trials by means of a button press with their right hand. However, since the number of catch trials was equal in each condition, the difference between conditions is surprising. Still, nicotine deprivation may have caused restlessness and neural responses related to motion preparation in smokers, and this could have led to unnecessary button presses to both types of cues. It is possible that, in the satiated condition more unnecessary movements were triggered by the smoking related than the neutral pictures. Therefore we suggest that better decoding of smoking related vs neutral cues in left precentral gyrus when participants were 
satiated may be related to motor responsiveness of the right hand in reaction to smoking related compared to neutral stimuli.

Still, a few considerations should be taken into account when interpreting our findings. First, our participants exhibited only low to moderate levels of nicotine dependency, as indicated by their low FTND scores. Therefore it is possible that the smoking cues were not as attractive or attention grabbing for them as they would have been for highly dependent individuals. Hence for more dependent smokers, patterns of activity related to smoking cues may have been better discriminable from those related to neutral cues. Moreover, highly dependent smokers would have been more affected by the nicotine deprivation condition, possibly increasing discriminability between smoking and neutral cues in the deprived condition even more. In addition, our deprivation condition was not successful in inducing craving. This could be due to a too short -and overnight- abstinence period, during which participants only missed a few cigarettes. For instance, 16 hours of abstinence have been shown to reliably induce craving (Jarvik et al., 2000), while our participants were only abstinent for 12 hours on average. Hence, longer periods of abstinence (in heavier smokers) may lead to more distinct responses to smoking related and neutral cues in additional neural regions. Secondly, to control as much as possible for other influences, we have kept our images of smoking related and neutral objects very 'clean'. They consisted of just one object in a neutral colour presented centrally on a white background. This may have made our smoking images less attractive and interesting to look at than more lifelike images of smoking scenes in which people enjoy smoking cigarettes. Therefore, more natural smoking related images could possibly have elicited neural responses that would be more differential from those to neutral stimuli.

In conclusion, we have used multi-voxel pattern analysis and a multivariate searchlight approach to elucidate brain regions that discriminate between smoking related and neutral cues during states of satiety or deprivation. We identified bilateral lateral occipital regions showing better decoding of visually similar smoking related vs neutral stimuli when participants were deprived of nicotine. These findings support the results of our previous study, indicating that the mechanism by which a smoker's attention is biased towards smoking cues indeed affects processing in the early visual system. 


\section{References}

Benowitz, N. L. (2010). Nicotine addiction. The New England Journal of Medicine, 362, 2295-303.

Breman, H., Peters, J., Weiskopf, N., Ashburner, J., \& Goebel, R. (2009). Fast fieldmapbased EPI distortion correction with anatabacus: a plugin for BrainVoyager. In NeuroImage (Vol. 47, p. S81).

Cappelleri, J. C., Bushmakin, A. G., Baker, C. L., Merikle, E., Olufade, A. O., \& Gilbert, D. G. (2005). Revealing the multidimensional framework of the Minnesota nicotine withdrawal scale. Current Medical Research and Opinion, 21(5), 749-60.

Conklin, C. A., Vella, E. J., Joyce, C. J., Salkeld, R. P., Perkins, K. A., \& Parzynski, C. S. (2015). Examining the relationship between cue-induced craving and actual smoking. Experimental and Clinical Psychopharmacology, 23(2), 90-96.

David, S. P., Munafò, M. R., Johansen-Berg, H., Mackillop, J., Sweet, L. H., Cohen, R. A., ... Walton, R. T. (2007). Effects of Acute Nicotine Abstinence on Cue-elicited Ventral Striatum/Nucleus Accumbens Activation in Female Cigarette Smokers: A Functional Magnetic Resonance Imaging Study. Brain Imaging and Behavior, 1(3-4), $43-57$.

David, S. P., Munafò, M. R., Johansen-Berg, H., Smith, S. M., Rogers, R. D., Matthews, P. M., \& Walton, R. T. (2005). Ventral striatum/nucleus accumbens activation to smoking-related pictorial cues in smokers and nonsmokers: a functional magnetic resonance imaging study. Biological Psychiatry, 58(6), 488-94.

De Martino, F., Valente, G., Staeren, N., Ashburner, J., Goebel, R., \& Formisano, E. (2008). Combining multivariate voxel selection and support vector machines for mapping and classification of fMRI spatial patterns. NeuroImage, 43(1), 44-58.

Due, D. L., Huettel, S. A., Hall, W. G., \& Rubin, D. C. (2002). Activation in mesolimbic and visuospatial neural circuits elicited by smoking cues: evidence from functional magnetic resonance imaging. The American Journal of Psychiatry, 159(6), 954-60.

Engelmann, J. M., Versace, F., Robinson, J. D., Minnix, J. a, Lam, C. Y., Cui, Y., ... Cinciripini, P. M. (2012). Neural substrates of smoking cue reactivity: a metaanalysis of fMRI studies. NeuroImage, 60(1), 252-62.

Field, M., \& Cox, W. M. (2008). Attentional bias in addictive behaviors: a review of its development, causes, and consequences. Drug and Alcohol Dependence, 97(1-2), 1- 
20.

Field, M., Marhe, R., \& Franken, I. H. A. (2014). The clinical relevance of attentional bias in substance use disorders. CNS Spectrums, 19(03), 225-230.

Franken, I. H. A. (2003). Drug craving and addiction: integrating psychological and neuropsychopharmacological approaches. Progress in Neuropsychopharmacology and Biological Psychiatry, 27(4), 563-79.

Franklin, T. R., Wang, Z., Wang, J., Sciortino, N., Harper, D., Li, Y., ... Childress, A. R. (2007). Limbic activation to cigarette smoking cues independent of nicotine withdrawal: a perfusion fMRI study. Neuropsychopharmacology, 32(11), 2301-2309.

Havermans, A., Vuurman, E. F. P. M., van den Hurk, J., Hoogsteder, P., \& van Schayck, O. C. P. (2014). Treatment with a nicotine vaccine does not lead to changes in brain activity during smoking cue exposure or a working memory task. Addiction, 109(8), $1260-7$.

Heatherton, T. F., Kozlowski, L. T., Frecker, R. C., \& Fagerström, K. O. (1991). The Fagerström Test for Nicotine Dependence: a revision of the Fagerström Tolerance Questionnaire. British Journal of Addiction, 86, 1119-1127.

Hester, R., \& Luijten, M. (2013). Neural correlates of attentional bias in addiction. CNS Spectrums, (August), 1-8.

Janes, A. C., Frederick, B. deB., Richardt, S., Burbridge, C., Merlo-Pich, E., Renshaw, P. F., ... Kaufman, M. J. (2009). Brain fMRI reactivity to smoking-related images before and during extended smoking abstinence. Experimental and Clinical Psychopharmacology, 17(6), 365-373.

Janes, A. C., Pizzagalli, D. A., Richardt, S., Frederick, B. deB., Chuzi, S., Pachas, G., ... Kaufman, M. J. (2010). Brain reactivity to smoking cues prior to smoking cessation predicts ability to maintain tobacco abstinence. Biological Psychiatry, 67(8), 722729.

Janes, A. C., Pizzagalli, D. A., Richardt, S., Frederick, B. deB., Holmes, A. J., Sousa, J., ... Kaufman, M. J. (2010). Neural substrates of attentional bias for smoking-related cues: an FMRI study. Neuropsychopharmacology, 35(12), 2339-2345.

Jarvik, M. E., Madsen, D. C., Olmstead, R. E., Iwamoto-Schaap, P. N., Elins, J. L., \& Benowitz, N. L. (2000). Nicotine Blood Levels and Subjective Craving for Cigarettes. Pharmacology Biochemistry and Behavior, 66(3), 553-558. 
Luijten, M., Veltman, D. J., van den Brink, W., Hester, R., Field, M., Smits, M., \& Franken, I. H. A. (2011). Neurobiological substrate of smoking-related attentional bias. NeuroImage, 54(3), 2374-81.

McBride, D., Barrett, S. P., Kelly, J. T., Aw, A., \& Dagher, A. (2006). Effects of expectancy and abstinence on the neural response to smoking cues in cigarette smokers: an fMRI study. Neuropsychopharmacology, 31, 2728-2738.

McClernon, F. J., Hiott, F. B., Huettel, S. A., \& Rose, J. E. (2005). Abstinence-induced changes in self-report craving correlate with event-related FMRI responses to smoking cues. Neuropsychopharmacology, 30(10), 1940-7.

McClernon, F. J., Kozink, R. V, Lutz, A. M., \& Rose, J. E. (2009). 24-h smoking abstinence potentiates fMRI-BOLD activation to smoking cues in cerebral cortex and dorsal striatum. Psychopharmacology, 204(1), 25-35.

Mourão-Miranda, J., Bokde, A. L. W., Born, C., Hampel, H., \& Stetter, M. (2005). Classifying brain states and determining the discriminating activation patterns: Support Vector Machine on functional MRI data. NeuroImage, 28(4), 980-95.

Nestor, L., McCabe, E., Jones, J., Clancy, L., \& Garavan, H. (2011). Differences in "bottom-up" and "top-down" neural activity in current and former cigarette smokers: Evidence for neural substrates which may promote nicotine abstinence through increased cognitive control. NeuroImage, 56(4), 2258-2275.

Pessoa, L., \& Ungerleider, L. G. (2004). Neuroimaging studies of attention and the processing of emotion-laden stimuli. Progress in Brain Research, 144, 171-182.

Robinson, T. E., \& Berridge, K. C. (2008). The incentive sensitization theory of addiction: some current issues. Philosophical Transactions of the Royal Society of London. Series B, Biological Sciences, 363(July), 3137-3146.

Waters, A. J., Shiffman, S., Sayette, M. A., Paty, J. A., Gwaltney, C. J., \& Balabanis, M. H. (2003). Attentional bias predicts outcome in smoking cessation. Health Psychology, 22(4), 378-87. 
Neural and cognitive determinants of smoking addiction and cessation 


\section{CHAPTER 4}

How to win -and not only fight- a running battle:

Web-based attention bias modification training for active smokers

Havermans, A., Vuurman, E.F.P.M., Riedel, W.J., \& van Schayck C.P. How to win -and not only fight- a running battle: Web-based attention bias modification training for active smokers. (submitted). 


\begin{abstract}
Various treatments have become available for smoking cessation, however success rates remain low. One underexposed factor in the maintenance of smoking behavior is the influence of smoking related environmental cues. We aimed to decrease smokers' attention bias towards such cues by means of a web-based attention bias modification (ABM) training. Smokers aged 18-60 years were randomly allocated to ABM $(n=208)$ or a control condition $(n=190)$. Both ABM and control trainings were based on a dot-probe task. Faster reaction times to probes that followed smoking related pictures reflected an attention bias. In the ABM training $80 \%$ of the probes followed neutral pictures. Participants completed five training sessions on (semi-) consecutive days. Attention bias was assessed one day after the final training and after one week follow-up. In the assessment we added a new subset of images to examine whether the effect of the training was generalizable. After five sessions of online training, attention bias was reversed in the participants who had received the intervention training $(n=70)$, but not for those in the control training $(n=61$; mean diff $=6.010,95 \%$ CI $\left.[1.03,10.99], \mathrm{F}_{(1)}=5.648, \mathrm{p}<.02, \eta^{2}=0.022\right)$. Moreover this effect was still present at follow-up one week later, and was generalizable to novel smoking related images. Based on these findings we conclude that attention bias modification is effective in reducing attention bias to smoking cues and may therefore be of great help in preventing relapses.
\end{abstract}




\section{Introduction}

Up to date, several pharmacological substances are available to aid in smoking cessation. In general they target the physiological effects of nicotine, which are often regarded as a crucial precipitating factor in tobacco addiction (Benowitz, 2010). Besides pharmacological treatments, behavioral therapy and counseling can offer additional benefits for people who wish to quit smoking. Such non-pharmacological therapies are mostly aimed at helping smokers to cope with psychological aspects associated with tobacco dependence(Fiore et al., 2008). Nevertheless, even with the most successful combination of behavioral support and medication, not even half of the (ex-)smokers manage to remain abstinent for at least one year (Rigotti, 2013).

So generally, currently available smoking cessation therapies are aimed at merely two aspects (i.e. the physiological effects of nicotine, and the psychological consequences of the addiction) of the multi-facetted character of tobacco addiction. A typically unaccounted for, but important factor in the maintenance of tobacco addiction is the interaction between neural events and the smokers' environment; i.e. after repeated smoking, drug related cues in the environment become associated with the rewarding physiological effects of nicotine. For long-term smokers these cues can become highly salient and craving-eliciting (Benowitz, 2010; Robinson \& Berridge, 2008). Moreover, smokers are known to automatically focus and maintain their attention on these smokingrelated cues in their environments; a process termed attention bias (Field \& Cox, 2008). Cue-elicited craving and attention bias have both been implicated in provoking relapses and the maintenance of smoking addiction (Ferguson \& Shiffman, 2009; Field, Munafò, \& Franken, 2009; Andrew J. Waters et al., 2003). Additionally, biased attention and subjective craving in response to smoking cues increase when smokers are deprived of nicotine (Field, Mogg, \& Bradley, 2004; A.J. Waters \& Feyerabend, 2000), like during smoking cessation attempts. The automatic focus on craving-provoking environmental cues thus seems to be an impeding factor for smokers who wish to quit. And (pharmacological) interventions are likely to be less effective as long as smokers keep encountering the cues in their environment that trigger craving. This process is a lot like fighting a running battle. Making smokers fight this running battle endlessly is pointless; instead we should help them win it. Strikingly, the influence of smoking related cues and attention bias has, until recently, not received full attention in the development of cessation treatments.

Avoiding exposure to smoking related cues would prevent cue-related craving, but given the abundance of smoking related stimuli in the environment this is not realistic. A more suitable therapeutic approach should aim at desensitizing smokers for these environmental cues, either through extinction of automatic attentional orienting or disengagement. Following such an approach, research in other fields has shown that 
attention bias can be attenuated and that this can have beneficial effects on disorder-specific symptoms (Schmidt, Richey, Buckner, \& Timpano, 2009; Schoenmakers et al., 2010; Schoenmakers, Wiers, Jones, Bruce, \& Jansen, 2007). The most commonly used attention bias modification (ABM) paradigm is a modified version of the dot probe task (MacLeod, Rutherford, Campbell, Ebsworthy, \& Holker, 2002). In this task, participants are simultaneously presented with two stimuli; one of which disorder-related, the other one neutral. As the stimuli disappear, one of them is followed by a probe, and participants are instructed to indicate its location as quickly as possible. In the standard version of the task, the probe appears in an equal number of trials in the location of the disorder related cue and the neutral cue. In this case, shorter reaction times to probes that follow disorder-related cues reflect an attention bias towards it. However, by manipulating this task such that the probe appears in all or most cases in the location of the neutral stimulus, participants can be implicitly conditioned to allocate their attention away from the disorder-related stimulus. Several studies were successful in decreasing attention bias for threatening stimuli by means of this task and as a result showed reductions in subjective anxiety. Although the magnitude and clinical significance of this effect are still under debate, ABM is generally endorsed as a treatment for anxiety disorders (Kuckertz \& Amir, 2015; Mogoaşe, David, \& Koster, 2014). Studies of ABM in substance use disorders are scarcer and provide insufficient evidence to support clinical effectiveness. Nevertheless, a few studies have promising results. For instance, Schoenmakers and colleagues demonstrated decreased attention bias to alcohol-related cues for heavy drinkers as well as for clinical alcohol dependent patients (Schoenmakers et al., 2010, 2007). Moreover, patients who received ABM training in addition to traditional behavioral therapy were discharged earlier form the clinic than patients who received a control training next to their therapy (Schoenmakers et al., 2010).

Three studies have investigated the effects of a single session ABM for smokers. They were moderately successful in attenuating the attention bias towards smoking cues, but did not produce robust changes in craving or smoking behavior (Attwood, O'Sullivan, Leonards, Mackintosh, \& Munafò, 2008; Field, Duka, Tyler, \& Schoenmakers, 2009; McHugh, Murray, Hearon, Calkins, \& Otto, 2010). Another three recent trials investigated the effects of $\mathrm{ABM}$ for smokers over multiple sessions. One study did not find any effects of five sessions of $\mathrm{ABM}$ in addition to standard smoking cessation support to smokers (Begh et al., 2015). However, these results should be interpreted with caution, since there was also no evidence of attentional bias prior to the treatment. Another study, which did demonstrate attention bias to smoking cues at baseline, showed effectiveness of three but not less sessions of ABM in reducing attention bias for up to six months after treatment (Lopes, Pires, \& Bizarro, 2014). Moreover, number of cigarettes smoked/day, CO and level 
of nicotine dependence were reduced in all treatment groups. Hence, this was not a consequence of ABM specifically but more likely was caused by the smoking cessation program participants were enrolled in. The third study adopted an ecological momentary assessment approach by delivering ABM in participants' natural environment on portable devices at three random times during the day (Kerst \& Waters, 2014). Participants received 15 sessions in five days, which significantly reduced attention bias. Moreover, ABM reduced craving in response to a compound cue, representing both smoking-related and neutral content.

Altogether these studies give rise to many questions regarding the efficacy of $\mathrm{ABM}$ in smokers. It is likely that implicit automatic attentional processing can be targeted by means of ABM; however various factors seem to impact on its success. Moreover, it is not yet clear if, and in which conditions, $\mathrm{ABM}$ can influence craving. In order to improve its effectiveness, several features of ABM should be considered. First of all, it is clear that multiple sessions of $\mathrm{ABM}$ are more effective than just a single session. Secondly, the effect of ABM needs to be stabile over a longer period of time, and not just disappear right after training. In order to assess this, an ABM trial should encompass at least one follow-up session. Moreover, since the environmental cues in a smoker's life will continually keep changing, it is important that the effect of ABM training is generalizable to other cues than just the ones used in the training. Also, assessing this generalizability is necessary to distinguish simple task-practice effects from an actual modification of implicit cognitive processes. Finally, providing training in the participants' natural environment instead of a research lab may be beneficial (Kerst \& Waters, 2014), because of easy administration of multiple trainings in a limited period of time, less effort for participants and increased ecological validity.

Considering these features, we have developed an online ABM training for smokers, consisting of five training sessions that were to be completed on (semi-) consecutive days. Attention bias and craving will be measured one day before the first and after the final training. In addition we have added a follow-up measurement to examine whether the effect of the ABM training will be maintained for one week, without training. Moreover, we will assess attention bias using the same images as during the training, as well as using novel images. We are the first to provide such an elaborate ABM trial for smokers fully web-based. Providing this training online, allows us to reach a large sample of smokers that well reflects the composition of the general population of smokers. Moreover, online treatment may offer increased convenience, confidentiality, and reduced stigma for smokers (Brown et al., 2014). We hypothesize that our training will diminish attention bias and potentially subjective craving in active smokers and that this effect will 
still be present at follow-up one week later. Moreover we expect that this effect will not just be present for the trained smoking cues, but will also be generalizable to novel ones. 


\section{Methods}

\section{Participants}

Eligible participants for our study were current smokers between the ages of 18 and 60 years. They were not informed about possible (beneficial) effects of participation on their smoking behavior, or about the study's potential application in smoking cessation. Participants were recruited by local poster advertisements in Maastricht University and online advertisement on various nationwide as well as international websites. The study was approved by the local ethics committee and participants indicated their informed consent online, prior to participation. Five monetary rewards were raffled between the participants who completed all eight sessions.

\section{Procedure}

The study consisted of eight sessions in total; a baseline measurement, five (ABM or control) trainings, a post-training assessment, and a follow-up. Participants were instructed to perform the first seven sessions each on separate days, with a maximum of two days between each session. The final follow-up session took place one week after the preceding session. At the start of the first session demographic characteristics (age, gender, and education), smoking history and the participants' motivation to quit were assessed. Moreover, we applied a single item craving rating (West \& Ussher, 2010) during the first and last session. All data was collected online, over a period of four months.

\section{Stimuli}

Pairs of smoking related and neutral images measuring 250×290 pixels were presented centrally on the computer screen (size of screen varied due to the online nature of the task). Smoking cues depicted scenes of people smoking as well as more detailed images of for instance a hand holding a cigarette. Neutral images were matched to the smoking related pictures based on colors and content. Moreover, the luminance of each picture pair was equalized using custom written code in MATLAB R2014a (Mathworks Inc., Natick, MA).

\section{Assessment dot-probe task (session 1,7, and 8)}

In the assessment-phase of the dot-probe task 36 smoking/neutral picture pairs were presented four times, equaling 144 trials. Each trial consisted of: a fixation period (duration: 400 or $800 \mathrm{~ms}$ ), presentation of the two pictures (duration: 400 or $600 \mathrm{~ms}$ ), and the dot (maximum response time: $2000 \mathrm{~ms}$ ), see figure 1. The design was semi-randomized such that the eight possible combinations of durations and dot- and picture locations occurred 18 times each (see supplementary data table $1 a$ and $1 b$ ). The dot replaced the smoking related picture and the neutral picture each in $50 \%$ of the trials, so each in nine of the 18 
occurrences of each trial type. The very first dot-probe assessment started with 12 practice trials which were not included in the analyses.

Training dot-probe task (session 2-6)

The dot-probe training task consisted of 12 repetitions of 26 picture pairs, equaling 312 trials. For the participants in the ABM training condition, the dot replaced the neutral cue in $80 \%$ of the trials. However, for the participants receiving the control training, which was expected not to influence their attention in any direction, the dot replaced the neutral and smoking related images both in $50 \%$ of the trials. Besides that, the durations and the design were similar to those of the assessment dot-probe task (see supplementary data table $1 \mathrm{~b}$ ).

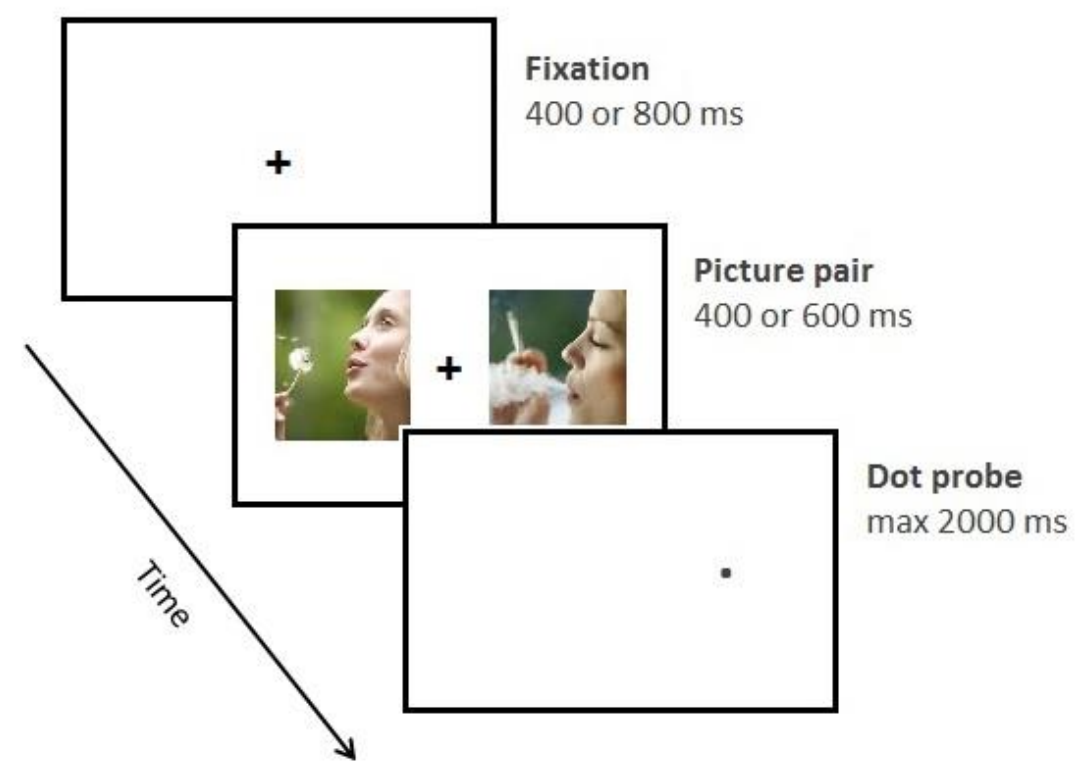

Figure 1. Overview of a dot-probe trial.

\section{Data-reduction}

Data of participants who had at least completed seven sessions $(n=134$, see figure 1$)$ were used for analyses. Missed and incorrect trials (3.44\%) were removed from the data, as well as reaction times shorter than $150 \mathrm{~ms}$ and longer than $2000 \mathrm{~ms}(0.59 \%)$. Two participants, for whom less than $75 \%$ of their data remained after these reductions, were excluded from the analyses, as were the complete first sessions of five other participants. Since the 
experiment was not conducted under controlled circumstances in a research lab, we aimed to reduce the influence of external factors on the data by removing outlying values for each individual participant in each measurement. To identify and remove individual outliers (2.78\% of the whole dataset) custom written code in MATLAB R2014a (Mathworks Inc., Natick, MA) was used that employed the outlier labeling rule, first introduced by Tuckey and later adjusted by Hoaglin and Iglewicz (Hoaglin \& Iglewicz, 1987).

\section{Analyses}

Statistical analyses were performed using IBM SPSS Statistics for Windows, version 21.0 (IBM Corp., Armonk, NY). Independent samples t-tests were used to compare the training groups at baseline regarding age of participants, and level of nicotine dependence (FTND). In addition, chi-square tests were applied to compare the groups on the ratio of male to females, education, and number of cigarettes smoked per day. Attention bias scores were obtained for each participant, separately for each measurement and picture set (trained and untrained) by subtracting the median reaction times to dots replacing smoking pictures from the median reaction times to dots replacing neutral pictures. This way, a positive score reflects an attention bias towards smoking cues. To examine whether our participants exhibited an attention bias before the start of the training, a one-sample t-test was employed on the scores at baseline, followed by an independent samples t-test to test for a difference between the training groups. Moreover, in order to determine the effects of ABM, post-test AB-scores were analyzed using a $2 \times 2 \times 2$ analysis of covariance (ANCOVA), with baseline attention bias scores as covariate to increase statistical power (Van Breukelen, 2006), a between-subjects factor of training and within-subjects factors of measurement (post, follow-up) and picture set. Finally the long-term effects of ABM were tested by means of another $2 \times 2 \times 2$ analysis of covariance (ANCOVA), with baseline- and post-test attention bias scores as covariates, the between-subjects factor training and measurement (post, follow-up) and picture set as within-subjects factors. Furthermore the effect of ABM on craving was analyzed by means of a 2 x 2 ANCOVA with baseline scores as covariate and the between-subject factor of training group. Finally we performed a correlational analysis to investigate the relationship between craving and attention bias at each timepoint. 


\section{Results}

\section{Participants}

A complete diagram of participant flow is depicted in figure 2. Characteristics of participants from both training groups are displayed in table 1 . The groups did not differ significantly on any of the demographic and smoking related measures.

\section{Table 1}

Characteristics of participants and craving and $\mathrm{AB}$ scores at baseline

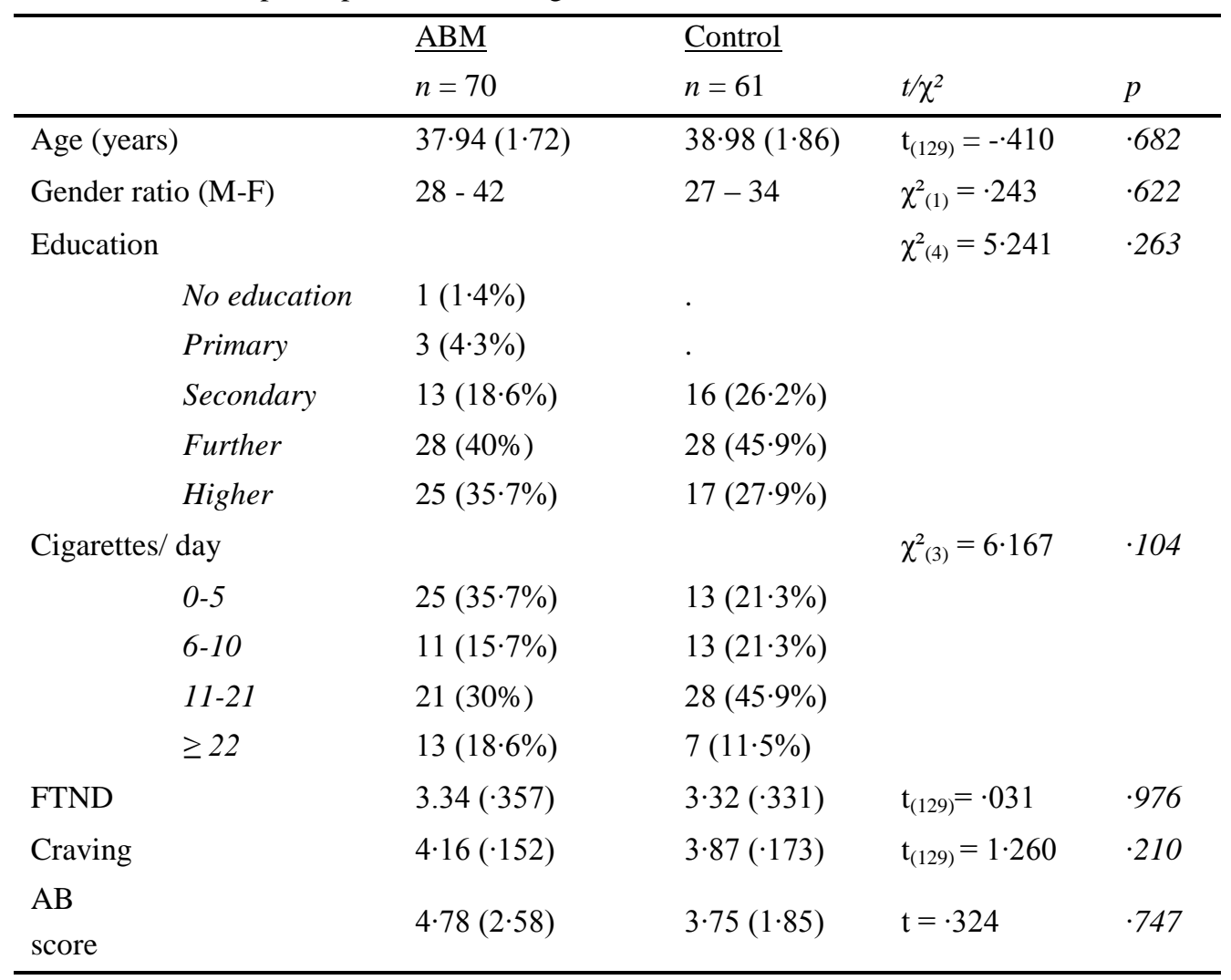

Note. Values are means $( \pm$ SEM).

Values for education and Cigarettes/ day are $\mathrm{n}(\%$ of the total) 


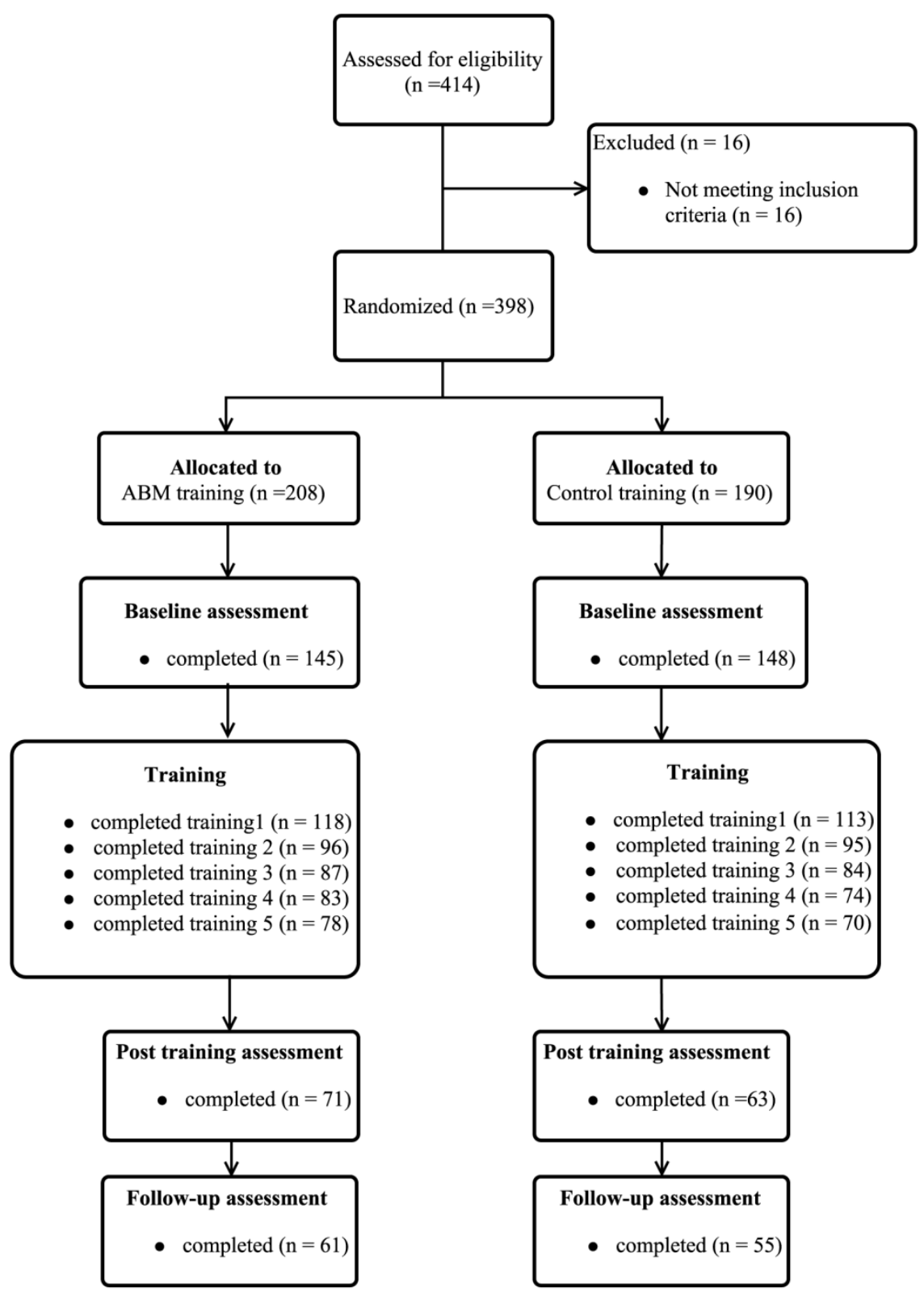

Figure 2. Flowchart of participants. 


\section{Attention bias}

Mean attention bias scores at each measurement and for both picture sets are displayed in table 2. At the baseline measurement participants showed an attention bias towards smoking related cues $\left(\right.$ mean $\left.=3.88, \mathrm{t}_{(252)}=2.599, \mathrm{p}<.05\right)$. Besides, there was no significant difference in attention bias scores between the intervention and control groups (mean diff $\left.=1.504, \mathrm{t}_{(250)}=501, \mathrm{p}=.617\right)$. There was a significant main effect of training on the posttraining $\mathrm{AB}$ scores $\left(\mathrm{F}_{(1,247)}=5.648, \mathrm{p}<.05\right.$. $)$. Posthoc pairwise comparisons revealed that attention bias for smoking cues after five sessions of training was significantly lower in the ABM group compared to the control group (mean diff $=6.010,95 \%$ CI $[1.03,10.99]$, $\eta^{2}=0.022$ ). Moreover, there was also a significant main effect of training on the follow-up $\mathrm{AB}$ scores $\left(\mathrm{F}_{(1,234)}=4.634, \mathrm{p}<.05\right.$.). Again, attention bias for smoking cues was significantly lower in the ABM group compared to the control group (mean diff $=5.794$, $95 \%$ CI $[0.50,11.09], \eta^{2}=.018$ ). There was no significant effect of pictureset on attention bias, at any of the measurement timepoints. For a graphical reproduction of the attention bias scores at each measurement, see figure 3 .

\section{Table 2}

$\mathrm{AB}$ scores at baseline, post-test and follow-up for novel and trained picture sets.

$$
\operatorname{ABM}(\mathrm{N}=70) \quad \text { Control }(\mathrm{N}=61)
$$

\begin{tabular}{lll}
\hline \hline Baseline & $4.58(2.17)$ & $3.07(2.02)$ \\
Post-test & $-1.58(1.76)$ & $3.92(1.75)$ \\
Novel & $1.03(2.45)$ & $1.27(2.76)$ \\
Trained & $-4.18(2.51)$ & $6.57(2.13)$ \\
Follow-up & $0.83(1.84)$ & $6.75(1.86)$ \\
Novel & $1.63(3.12)$ & $8.37(3.30)$ \\
Trained & $.03(1.96)$ & $5.14(1.72)$ \\
\hline
\end{tabular}

Note. Values are means ( \pm SEM). 


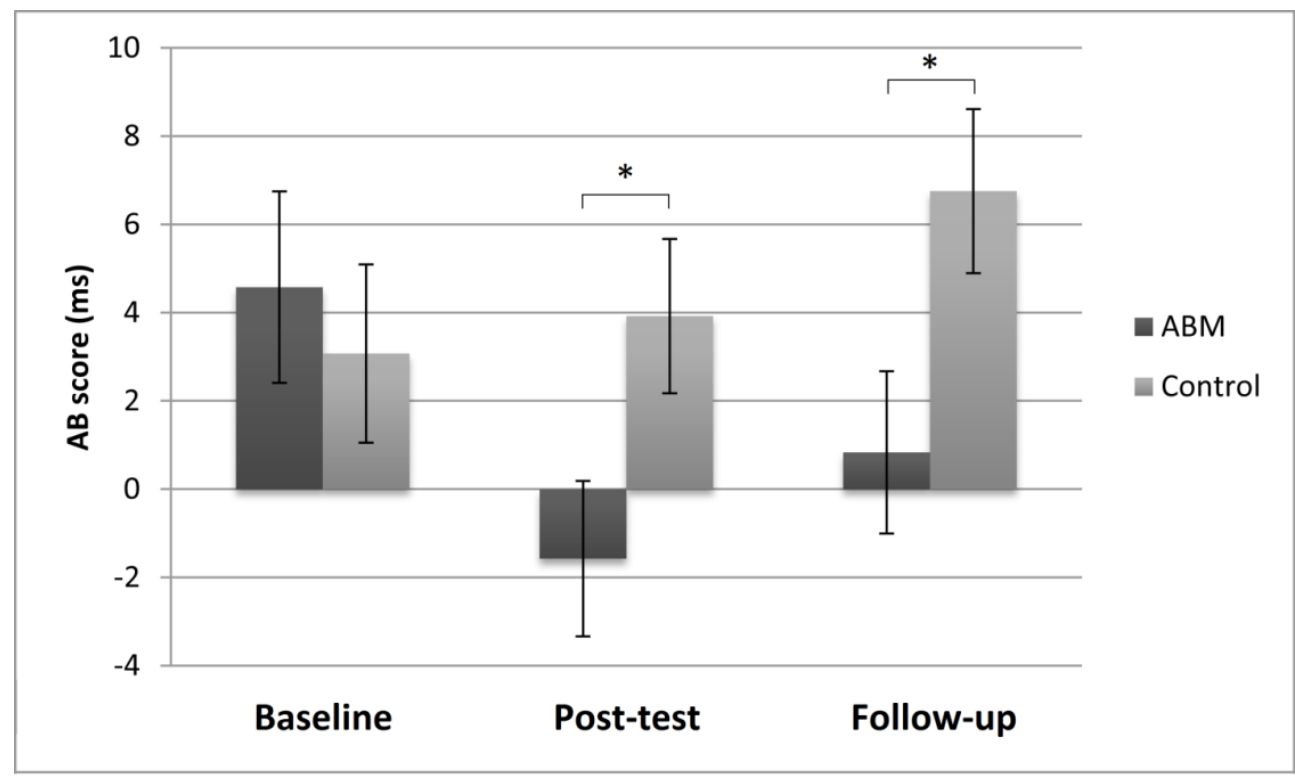

Figure 3. Mean attention bias (AB) scores at each measurement, over both picture sets. 


\section{Craving}

The mean craving score was significantly lower at the post-test (mean diff $=.54, \mathrm{t}_{(130)}$ $=4.238, \mathrm{p}<.001$ ) and follow-up (mean diff $=.31, \mathrm{t}_{(128)}=2.618, \mathrm{p}<.02$ ) assessments, than at baseline. However there was no significant difference in craving between the groups at both time points (post-test mean diff $=.019, \mathrm{~F}_{(1)}=.007, \mathrm{p}=.932$; follow-up mean diff $=.155, \mathrm{~F}_{(1)}$ $=.739, \mathrm{p}=.391)$. The craving scores for both training groups are graphically represented in figure 4. Craving scores did not significantly correlate with attention bias at baseline $(\mathrm{r}=.023, \mathrm{~ns})$, post-training assessment (r=-.004, ns), or follow-up (r=-.036, ns).

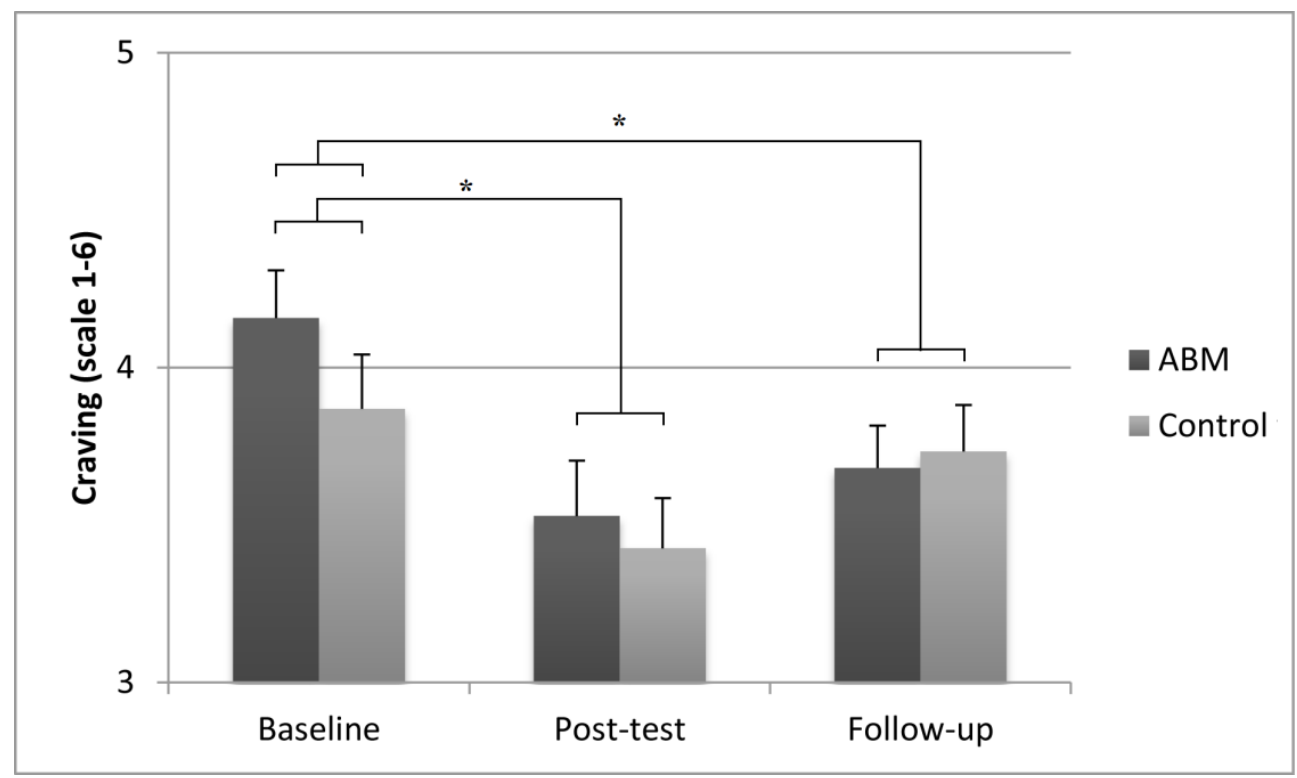

Figure 4. Craving scores at each measurement. 


\section{Discussion}

This online attention bias modification training for smokers was aimed at reducing the urge to smoke, by decreasing one of its underlying mechanisms; attention bias to smoking cues. As hypothesized, our participants, who were all smokers, exhibited an attention bias towards smoking-related pictures at baseline. After five sessions of training, this attention bias was reversed in the participants who had received the ABM training, but not for those in the control training. Moreover this effect was still present at follow-up one week later, without any additional training. Furthermore, this decreased attention bias was not just demonstrated for the smoking pictures that were used in the training, but also for completely novel ones. This means that the effect is not only robust, but also generalizable to different situations.

Our study has shown that the mechanism of ABM is effective in reducing attention bias; however it is unclear whether this will lead to changes in smoking behavior. We did not find an effect of multiple sessions of ABM on overall subjective craving levels, which is in line with findings of other ABM studies that have been published since the start of our study (Begh et al., 2015; Kerst \& Waters, 2014; Lopes et al., 2014). Moreover, craving scores did not significantly correlate with attention bias, indicating that attention bias is not related to the more general level of subjective craving as was measured with our craving related questionnaire. Therefore it is likely that targeting implicit attentional processing with ABM also does not influence this general craving. However, ABM may still influence craving after exposure to smoking related cues, as trained smokers should automatically direct less attentional processing resources to the smoking content and therefore experience less craving. Indeed, one of the studies that did not show an effect of ABM on overall craving did find reduced craving in response to smoking related cues after ABM.

Although studies in anxiety and alcohol dependence have shown clinical efficacy (Schmidt et al., 2009; Schoenmakers et al., 2010); up to now, no study has demonstrated an effect of ABM on measures of smoking behavior. Yet, ABM's aptitude to reduce attention bias to smoking cues is very promising, especially in relation to theoretical accounts suggesting a causal link between attention bias and smoking behavior (Field \& Cox, 2008). Moreover, attention bias to smoking cues has been associated with higher chances of lapses (Andrew J. Waters et al., 2003), whereas avoidance of smoking cues was associated with successful abstinence (Peuker \& Bizarro, 2013). Hence, decreasing attention bias by means of ABM may not directly affect smoking behavior in active smokers, but may prevent lapses after a quit attempt. Since ABM is thought to reduce craving in response to smoking cues, this could be a mechanism by which ABM influences ex-smokers' proneness to lapses. Future studies should aim to further investigate the application of ABM in relapse prevention. Additionally, effectiveness of ABM trainings for smokers may be improved, for 
instance by selecting stimuli for each participant individually or by combining the training with specific other types of psychological or pharmacological treatments.

The rate of attrition in our study is rather high (approximately 71\%), but does not differ between the treatment groups, suggesting that our results are not likely to be affected by attrition bias. High attrition rates are common in internet-based interventions (An et al., 2006), and are likely a consequence of various factors. First of all, the strict timing in the study -participants were required to complete each session within two days from the previous one- was difficult to keep up with. Moreover, several participants reported finding the task tedious and unexciting. In addition, there were no consequences for dropping out; participants were told that they were allowed to withdraw from the study without having to give up a reason, and they could do so from their own homes, without getting noticed or feeling discouraged by the researchers or others. Besides, since there was no personal contact with the researchers, participants may have lacked a certain feeling of responsibility towards them and the study.

This study was the first internet-based trial offering multiple sessions of ABM or control training to active smokers. Our results indicate that attention bias for smoking cues can be significantly attenuated by five semi-daily sessions of ABM training. Moreover, this effect was robust, as it remained present at one-week follow-up, and generalizable to novel smoking-related cues. In conclusion, ABM seems to be of crucial value to obtain and maintain avoidance of smoking cues after a quit attempt, and can thereby help prolonging abstinence. In this way, smokers will not have to keep fighting a running battle, but eventually they can win it. 


\section{Acknowledgements}

The authors would like to thank Michiel Vestjens and Charlie Bonnemayer for their contributions in programming the study software. 


\section{References}

An, L., Perry, C., Lein, E., Klatt, C., Farley, D., Bliss, R., ... Ehlinger, E. (2006). Strategies for increasing adherence to an online smoking cessation intervention for college students. Nicotine \& Tobacco Research, 8(1), 7-12.

Attwood, A. S., O’Sullivan, H., Leonards, U., Mackintosh, B., \& Munafò, M. R. (2008). Attentional bias training and cue reactivity in cigarette smokers. Addiction, 103(11), $1875-82$.

Begh, R., Munafò, M. R., Shiffman, S., Ferguson, S. G., Nichols, L., Mohammed, M. A., ... Aveyard, P. (2015). Lack of attentional retraining effects in cigarette smokers attempting cessation: A proof of concept double-blind randomised controlled trial. Drug and Alcohol Dependence, 149, 158-165.

Benowitz, N. L. (2010). Nicotine addiction. The New England Journal of Medicine, 362, 2295-303.

Brown, J., Michie, S., Geraghty, A. W. A., Yardley, L., Gardner, B., Shahab, L., ... West, R. (2014). Internet-based intervention for smoking cessation (StopAdvisor) in people with low and high socioeconomic status: a randomised controlled trial. Lancet. Respiratory Medicine, 2(12), 997-1006.

Ferguson, S. G., \& Shiffman, S. (2009). The relevance and treatment of cue-induced cravings in tobacco dependence. Journal of Substance Abuse Treatment, 36(3), 235243.

Field, M., \& Cox, W. M. (2008). Attentional bias in addictive behaviors: a review of its development, causes, and consequences. Drug and Alcohol Dependence, 97(1-2), 120.

Field, M., Duka, T., Tyler, E., \& Schoenmakers, T. M. (2009). Attentional bias modification in tobacco smokers. Nicotine \& Tobacco Research, 11(7), 812-22.

Field, M., Mogg, K., \& Bradley, B. P. (2004). Eye movements to smoking-related cues: effects of nicotine deprivation. Psychopharmacology, 173(1-2), 116-23.

Field, M., Munafò, M. R., \& Franken, I. H. A. (2009). A meta-analytic investigation of the relationship between attentional bias and subjective craving in substance abuse. Psychological Bulletin, 135(4), 589-607.

Fiore, M. C., Jaén, C. R., Baker, T. B., Bailey, W. C., Benowitz, N. L., Curry, S. J., ... 
Wewers, M. E. (2008). Treating tobacco use and dependence: 2008 update. Rockville(MD): US Department of Health and Human Services 2008.

Hoaglin, D. C., \& Iglewicz, B. (1987). Fine-Tuning Some Resistant Rules for Outlier Labeling. Journal of American Satistical Association, 82(1147-1149).

Kerst, W. F., \& Waters, A. J. (2014). Attentional retraining administered in the field reduces smokers' attentional bias and craving. Health Psychology, 33(10), 1232-40.

Kuckertz, J. M., \& Amir, N. (2015). Attention bias modification for anxiety and phobias: current status and future directions. Current Psychiatry Reports, 17(2), 9.

Lopes, F. M., Pires, A. V., \& Bizarro, L. (2014). Attentional bias modification in smokers trying to quit: a longitudinal study about the effects of number of sessions. Journal of Substance Abuse Treatment, 47(1), 50-7.

MacLeod, C., Rutherford, E., Campbell, L., Ebsworthy, G., \& Holker, L. (2002). Selective attention and emotional vulnerability: assessing the causal basis of their association through the experimental manipulation of attentional bias. Journal of Abnormal Psychology, 111(1), 107-23.

McHugh, R. K., Murray, H. W., Hearon, B. A., Calkins, A. W., \& Otto, M. W. (2010). Attentional bias and craving in smokers: the impact of a single attentional training session. Nicotine \& Tobacco Research, 12(12), 1261-4.

Mogoaşe, C., David, D., \& Koster, E. H. W. (2014). Clinical efficacy of attentional bias modification procedures: an updated meta-analysis. Journal of Clinical Psychology, 70(12), 1133-57.

Peuker, A. C., \& Bizarro, L. (2013). Attentional avoidance of smoking cues in former smokers. Journal of Substance Abuse Treatment.

Rigotti, N. A. (2013). Smoking cessation in patients with respiratory disease: existing treatments and future directions. Lancet. Respiratory Medicine, 1(3), 241-50.

Robinson, T. E., \& Berridge, K. C. (2008). The incentive sensitization theory of addiction: some current issues. Philosophical Transactions of the Royal Society of London. Series B, Biological Sciences, 363(July), 3137-3146.

Schmidt, N. B., Richey, J. A., Buckner, J. D., \& Timpano, K. R. (2009). Attention training for generalized social anxiety disorder. Journal of Abnormal Psychology, 118(1), 514. 
Schoenmakers, T. M., de Bruin, M., Lux, I. F. M., Goertz, A. G., Van Kerkhof, D. H. A. T., \& Wiers, R. W. (2010). Clinical effectiveness of attentional bias modification training in abstinent alcoholic patients. Drug and Alcohol Dependence, 109(1-3), 3036.

Schoenmakers, T. M., Wiers, R. W., Jones, B. T., Bruce, G., \& Jansen, A. T. M. (2007). Attentional re-training decreases attentional bias in heavy drinkers without generalization. Addiction, 102(3), 399-405.

Van Breukelen, G. J. P. (2006). ANCOVA versus change from baseline: more power in randomized studies, more bias in nonrandomized studies [corrected]. Journal of Clinical Epidemiology, 59(9), 920-5.

Waters, A. J., \& Feyerabend, C. (2000). Determinants and effects of attentional bias in smokers. Psychology of Addictive Behaviors, 14(2), 111-120.

Waters, A. J., Shiffman, S., Sayette, M. A., Paty, J. A., Gwaltney, C. J., \& Balabanis, M. H. (2003). Attentional bias predicts outcome in smoking cessation. Health Psychology, 22(4), 378-87.

West, R., \& Ussher, M. (2010). Is the ten-item Questionnaire of Smoking Urges (QSUbrief) more sensitive to abstinence than shorter craving measures? Psychopharmacology, 208(3), 427-432. 


\section{CHAPTER 5}

Predictors of successful attention bias modification in smokers

Havermans, A., Vuurman, E.F.P.M., van Schayck C.P., \& Riedel, W.J. Predictors of successful attention bias modification in smokers. (submitted). 


\begin{abstract}
Attention bias to craving-eliciting cues is thought to play an important role in the maintenance of smoking addiction. Recently, studies have shown promising effects of Attention Bias Modification (ABM) to reduce attention bias for smokers. However, the effects of $\mathrm{ABM}$ on craving and smoking behavior are less pronounced. Therefore, we examined whether the effectiveness of this training was influenced by specific characteristics of smokers. Smokers aged 18-60 years were randomly allocated to ABM $(n=208)$ or a control condition $(n=190)$. The trainings were based on a dot-probe task; faster reaction times to probes that followed smoking related pictures reflected an attention bias. Participants completed five training sessions on (semi-) consecutive days. Two separate factorial univariate analyses of variance (ANOVA) were applied to examine the effects of the different predictors on attention bias at baseline and post-training. We could not distinguish any predictors affecting attention bias at baseline. Moreover, no features significantly predicted attention bias after training, except for training type which indicates that $\mathrm{ABM}$ training was more effective than the control condition. These findings show that $\mathrm{ABM}$ may be equally beneficial for all smokers, which is very promising for the possible implementation of ABM in clinical practice.
\end{abstract}




\section{Introduction}

An important implicit cognitive process in the maintenance of addictions is attention bias. It has been frequently reported that substance dependent people automatically focus their attention on cues related to their addiction (Field \& Cox, 2008; Franken, 2003). These conditioned attentional responses develop when environmental cues are repeatedly paired with drug-related activation of the brain's reward pathway. Consequently, the cues start triggering craving and can become highly salient. The strength and direction of attention bias can vary depending on several factors, such as gender, personality traits and smoking habits (Hogarth, Mogg, Bradley, Duka, \& Dickinson, 2003; Perlato, Santandrea, Della Libera, \& Chelazzi, 2014). Attention bias to smoking-related cues has been demonstrated in heavy and light smokers, whereas former smokers showed attentional avoidance from smoking cues (Peuker \& Bizarro, 2013). Moreover, attention bias to smoking cues has been associated with higher chances of lapses after a quit attempt (Waters et al., 2003). Together these findings suggest that attention bias may play an important role in the maintenance of smoking addiction, and that reducing it may be crucial for successful smoking cessation.

Recently, researchers have been experimentally modifying attention bias to investigate its effects on craving and smoking behavior. Three studies have shown a decreased or even negative attention bias after multiple sessions of attention bias modification (ABM) training in smokers (Havermans et al., chapter 4; Kerst and Waters, 2014; Lopes et al., 2014). All three studies did not find an effect of ABM on general craving, but Kerst and Waters did report decreased craving in response to smoking cues. The studies that measured variables related to smoking behavior (i.e. exhaled CO, number of cigarettes/ day, nicotine dependence) did not find any ABM-related effects on these variables (Kerst \& Waters, 2014; Lopes et al., 2014). One other study did not report any differences in attention bias, craving or smoking behavior after training; however, they also did not find an attention bias to smoking cues at baseline (Begh et al., 2015). Altogether these studies have demonstrated the very promising ability of ABM to decrease attention bias; however they do not offer clear support for the benefit of ABM in reducing smoking behavior. In order to find out how the effectivity of ABM for smoking cessation can be improved, it is interesting to see whether there are factors influencing its effect. Since attention bias is likely affected by various participant-related features, this may also be the case for its malleability, and thus for ABM. In this study we aim to elucidate those participant-related factors that played a role in the effectiveness of our recent online ABM trial (chapter 4).

For this we will consider the following factors:

-Gender. There are confirmed sex-related differences in smoking behavior and addiction, for instance females become dependent faster, can stay abstinent for shorter episodes and 
relapse more than males (Pogun \& Yararbas, 2009). Moreover, females are more reactive to smoking cues and experience stronger craving in response (Carpenter et al., 2014; Doran, 2014; Field \& Duka, 2004). On the other hand, a recent study found that males showed larger attention biases towards smoking cues than women (Littel, Euser, Munafò, \& Franken, 2012). In addition, another study showed that successful augmentation of attention bias by means of ABM, led to increased cue-elicited craving only for the male participants (Attwood, O’Sullivan, Leonards, Mackintosh, \& Munafò, 2008).

-Dependency/ cigarette consumption. In spite of it being the topic of various investigations, the relationship between smoking behavior and attention bias is not quite clear yet. Some studies have demonstrated a larger attention bias in heavier smokers (Mogg \& Bradley, 2002; Vollstädt-Klein et al., 2011; Zack, Belsito, Scher, Eissenberg, \& Corrigall, 2001), whereas others found a larger attention bias in light smokers(e.g. Hogarth et al., 2003; Mogg et al., 2005). A reason for these mixed findings could be that attention bias was measured on trials with different stimulus onset asynchronies, while heavy smokers seem to exhibit a stronger attention bias towards smoking cues especially on trials with shorter stimulus onset asynchronies. Thus, the effect of dependency on attention bias may be influenced by timing of attentional processes.

-Motivation to quit. Participants' motivation to quit smoking is found to be predictive of future quit attempts (Zhou et al., 2009) and has also been related to successful smoking cessation (Caponnetto \& Polosa, 2008). Behavioral change in smoking addiction may be triggered especially by intrinsic motivation, driven by the anticipation of specific internal rewards such as improved health (Caponnetto \& Polosa, 2008). Conversely, ABM training does not involve a conscious change of behavior that could be elicited by intrinsic motivation. Nonetheless we aim to find out whether also unconscious cognitive processes such as attention bias are more impressionable in participants with a high motivation to quit, as opposed to those with a low motivation to quit.

-Education. Lower levels of education have been associated with higher tobacco dependence and may be related to lower motivation to quit (George \& Koob, 2010; Hiscock, Bauld, Amos, Fidler, \& Munafò, 2012; Pennanen et al., 2014; Siahpush, McNeill, Borland, \& Fong, 2006). Moreover, lower educated participants may have more difficulty adhering to the training schedule (Hiscock et al., 2012).

-Age. It is likely that participants of older age will respond slower and may have more difficulties with the dot-probe task. Moreover, one study found a larger attention bias in younger-aged males, only on trials with a short stimulus onset asynchrony (SOA) (Perlato et al., 2014).

-Motive for smoking. It is possible that attention bias differs between participants with different motives for continued smoking, such as enjoyment or stress-relief (Fidler \& West, 
2009). For instance, stress has been shown to increase attention bias to alcohol cues in social drinkers who drink to cope with negative sentiments (Field \& Powell, 2007; Field \& Quigley, 2009). Besides, social smokers or smokers who smoke when they drink may be more sensitized to smoking cues, as their episodes of smoking are more likely to be related to specific environments. Therefore these smokers may also be more sensitive to manipulation of attention bias.

In the current study we aim to find out how the above mentioned factors influence attention bias and ABM. We have recently conducted an internet-based multiple-session $\mathrm{ABM}$ trial in current smokers and our results showed that five sessions of ABM training reduced attention bias for smoking cues in a robust and generalizable way. As a continuation of that study we have applied a factorial analysis of variance in order to carefully examine the relationship between the smokers' characteristics and their attention bias at baseline, as well as the change in attention bias after ABM or control training. 


\section{Methods}

\section{Participants}

Current smokers between 18 and 60 years of age were recruted by means of poster advertisements in Maastricht University as well as online advertisement on nationwide and international websites. The study was approved by Ethics Committee Psychology of Maastricht University, and participants indicated their informed consent online, prior to participation. To increase motivation participants were given the option to enter a draw, after completing the study, in which five monetary incentives were distributed.

\section{Procedure}

This study followed the same procedure and methods as described in the previous study by Havermans and colleagues (chapter 4). Participants were randomly assigned to receive ABM or control training. The study consisted of five training sessions, a baseline-, posttraining, and follow-up measurement, totaling eight separate sessions. Participants were instructed to complete the first seven sessions each on separate days, with a maximum of two days between each two sessions. The follow-up session took place one week after the preceding session.

\section{Questionnaires}

At the start of the first session demographic characteristics (age, gender, and education) were assessed. Moreover, a Fagerström test for nicotine dependency was applied in order to assess cigarette consumption and dependence (Heatherton, Kozlowski, Frecker, \& Fagerström, 1991). Finally we asked participants to indicate their main motive for smoking out of four options (see table 1), and to rate their motivation to quit on a scale from one to ten.

\section{Stimuli}

Pairs of smoking related and neutral images measuring 250×290 pixels were presented centrally on the computer screen (size of screen varied due to the online nature of the task). Smoking cues depicted scenes of people smoking as well as more detailed images of for instance a hand holding a cigarette. Neutral images were matched to the smoking related pictures based on colors and content. Moreover, the luminance of each picture pair was equalized using custom written code in MATLAB R2014a (Mathworks Inc., Natick, MA).

\section{Dot-probe task}

Each task trial consisted of: a fixation period (duration: 400 or $800 \mathrm{~ms}$ ), presentation of two pictures (duration: 400 or $600 \mathrm{~ms}$ ), and a dot probe (maximum response time: $2000 \mathrm{~ms}$ ). 
The design was semi-randomized such that the all combinations of durations and dot- and picture locations occurred equally often. Participants were instructed to respond as quickly as possible to the location of the dot probe.

Assessment

In the assessment-phase of the dot-probe task 36 smoking/neutral picture pairs were presented four times, equaling 144 trials. The dot replaced the smoking related picture and the neutral picture each in $50 \%$ of the trials. The first dot-probe assessment started with 12 practice trials which were not included in the analyses.

\section{Training}

The dot-probe $\mathrm{ABM}$ training task consisted of 12 repetitions of 26 picture pairs, equaling 312 trials. For the participants in the ABM training condition, the dot replaced the neutral cue in $80 \%$ of the trials. However, for the participants receiving the control training, which was expected not to influence their attention in any direction, the dot replaced the neutral and smoking related images both in $50 \%$ of the trials.

\section{Analyses}

Statistical analyses were accomplished using IBM SPSS Statistics for Windows, version 21.0 (IBM Corp., Armonk, NY). Attention bias scores were obtained for each participant individually, for each measurement (pre, post, follow-up) by subtracting the median reaction times to dots replacing smoking pictures from the median reaction times to dots replacing neutral pictures. This way, a positive score reflects an attention bias towards smoking cues. Independent categorical variables were created based on the earlier introduced factors (see table 1). To examine the effects of the different predictors on baseline attention bias, a univariate analysis of variance (ANOVA) was applied, with all independent variables entered as between-subjects factors. For this analysis all main effects and second level interaction effects were entered in the model. The resulting model was then subjected to a stepwise backward fitting procedure, by removing non-significant interaction terms. Moreover, an analysis of covariance (ANCOVA) was performed with attention bias at post-test as dependent variable and attention bias at baseline as covariate, in order to increase statistical power (Van Breukelen, 2006). In this analysis, all features were added as between-subjects factors, and since we were interested to find out which of them influenced the ABM training effect, all main effects and second-level interactions with 'type of training' were added in the model. Again, this model was subjected to a stepwise backward fitting procedure, by removing non-significant interaction- and corresponding main terms. 


\section{Table 1.}

Independent factors in the analysis

\begin{tabular}{lll} 
Factor & Categories & $\mathrm{N}(\%)$ \\
\hline \hline Training & & \\
& $A B M$ & $70(53.4)$ \\
& Control & $61(46.6)$
\end{tabular}

Gender

$\begin{array}{ll}\text { Male } & 55(42) \\ \text { Female } & 76(58)\end{array}$

Cig/day

$\begin{array}{ll}<1 \text { per day } & 30(22.9) \\ 1-10 \text { per day } & 32(24.4) \\ 11-20 \text { per day } & 49(37.4) \\ >20 \text { per day } & 20(15.3)\end{array}$

Dependence (FTND score)

$\begin{array}{ll}\text { Very low }(0-2) & 58(44.3) \\ \text { Low }(3-4) & 23(17.6) \\ \text { Medium-high }(5-7) & 38(29.0) \\ \text { Very high }(8-10) & 12(9.2)\end{array}$

Motivation to quit

$\begin{array}{ll}\text { Low } & 34(26) \\ \text { Medium } & 53(40.5) \\ \text { High } & 44(33.5)\end{array}$

Education

Secondary or lower

Continued

Higher

$42(32.1)$

Age group
18-39 years
$62(47.3)$
40-60 years
$69(52.7)$

Motive for smoking

For pleasure $53(40.5)$ 
For stress-relief

In social situations

When drinking alcohol
$13(9.9)$

$8(6.1)$

$57(43.5)$ 


\section{Results}

\section{Attention bias}

At the baseline measurement participants showed an attention bias towards smoking related cues $\left(\right.$ mean $\left.=4.31, \mathrm{t}_{(125)}=2.645, \mathrm{p}<.009\right)$. At the posttest, this bias was reduced in the ABM training group compared to the control training group (mean diff $=6.010, \mathrm{~F}_{(1)}=5.648$, $\mathrm{p}<.02)$. The difference in attention bias between the training groups remained significant until the follow-up measurement, one week later (mean diff $=5.794, \mathrm{~F}_{(1)}=4.634, \mathrm{p}<.04$ ). For more details about the effectiveness of ABM training, see chapter 4.

\section{Influences on attention bias}

There was no significant effect of any of the factors on baseline attention bias. There was a significant effect of training type $\left(\mathrm{F}_{(1)}=4.328, \mathrm{p}<.05\right)$ on post-test attention bias, indicating that attention bias at post-test was lower in the group that received ABM training compared to the control group (mean difference $=5.823$ ). Moreover, the interaction between training type and cigarettes/day showed a trend towards significance $\left(F_{(3)}=2.442, p=.068\right)$. Simple effect analyses indicated that attention bias at post-test was significantly lower after ABM than control training, only in moderate smokers, smoking 11-20 cigarettes a day (mean difference $=11.41, \mathrm{t}_{(47)}=3.199, \mathrm{p}<003$, see figure 1$)$. Moreover, the difference in attention bias between the $\mathrm{ABM}$ and control group nearly reached significance in the lighter smokers, who smoke 1-10 cigarettes a day (mean difference $=9.9, \mathrm{t}_{(30)}=2.017, \mathrm{p}=.053$ ).

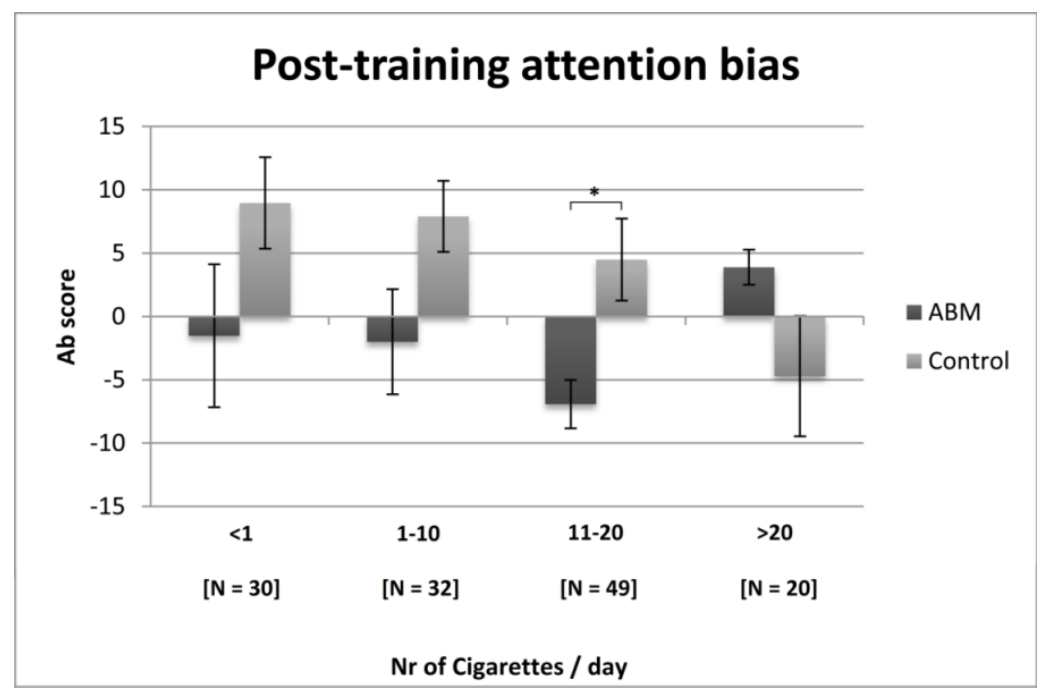

Figure 1. Interaction between training type and cigarettes/day on post-training attention bias. 


\section{Discussion}

In this study we aimed to find out which individual features play a role in attention bias and attention bias modification. To do this, we applied a factorial univariate analysis approach to the resulting data of our online multiple session ABM trial for smokers (chapter 4). We could not distinguish any predictors affecting attention bias at baseline. However, the final model for predicting attention bias at post-test showed a significant main effect of trainingtype and a trend towards significance for the interaction between training type and cigarettes/ day. The relationship between the type of training and post-test attention bias is clear, since attention bias was significantly diminished after the ABM training compared to the control training (chapter 4). The interaction effect indicates that ABM training may have been more succesful in reducing attention bias in moderate smokers, who smoke 1120 cigarettes a day. Simple effects analysis showed a significantly reduced attention bias in moderate smokers in the $\mathrm{ABM}$ training group. The finding that attention bias was not significantly more reduced by ABM than control in heavier smokers may be influenced by the small samplesize of that group. However, interpretation should be taken cautiously since the interaction effect was not significant.

The lack of effects on baseline attention bias is especially surprising, as many other studies have shown attention bias differs with gender, degree of tobacco dependence and age (Attwood et al., 2008; Hogarth et al., 2003; Littel et al., 2012; Mogg \& Bradley, 2002; Mogg et al., 2005; Perlato et al., 2014; Vollstädt-Klein et al., 2011; Zack et al., 2001). One possible explanation for the absence of an effect of these factors is that their effects may be modulated by time delay. For instance, Perlato and colleagues found that the best predictors for attention bias at SOA of $200 \mathrm{~ms}$ were age, gender, dependence and cigarette consumption (amongst others), whereas attention bias at SOA of $400 \mathrm{~ms}$ could best be predicted by dependency and cigarette consumption (amongst others) but not age and gender. However, at $800 \mathrm{~ms}$ the attention bias was only affected by gender and no other predictors (Perlato et al., 2014). In our study we did not take SOA into account while testing the influence of the different predictors on attention bias and post-training change in attention bias. We measured attention bias with a SOA of 400 or 600 milliseconds, so finding no influence of gender or age on attention bias is in agreement with the findings of Perlato et al (Perlato et al., 2014). However, there is still no explanation for not finding an effect of dependency or cigarette consumption.

The lack of significant predictors of ABM effects besides the one of training, suggests that ABM may be equally effective for all groups of smokers. This finding is very promising for the implementation of $\mathrm{ABM}$ as a clinical tool in smoking cessation. Since $\mathrm{ABM}$ can be applied in an online environment it is already easy to provide to smokers, on their own computers or even tablets or smartphones. Our current findings show that ABM 
may be equally beneficial for all smokers, so there seems to be no need to select specific candidates for this treatment. Therefore ABM can -and should- be made available widely as an aid in smoking cessation.

In conclusion, we aimed to elucidate how personal factors influence attention bias and its malleability. However, unlike previous studies (e.g. Perlato et al., 2014), we did not find any significant predictors for attention bias at baseline. Moreover, we could not identify any features significantly predicting attention bias after training, except for the obvious effect of training type. We did find a non-significant interaction effect, suggesting that $\mathrm{ABM}$ may be more successful in moderate, compared to light and heavy smokers. Altogether our results suggest that $\mathrm{ABM}$ may be equally effective for all smokers. This knowledge can contribute to uncomplicated clinical implementation of $\mathrm{ABM}$ for smoking cessation or relapse prevention. 


\section{Acknowledgements}

The authors would like to thank Michiel Vestjens and Charlie Bonnemayer for their contributions in programming the study software. 


\section{References}

Attwood, A. S., O’Sullivan, H., Leonards, U., Mackintosh, B., \& Munafò, M. R. (2008). Attentional bias training and cue reactivity in cigarette smokers. Addiction, 103(11), 1875-82.

Begh, R., Munafò, M. R., Shiffman, S., Ferguson, S. G., Nichols, L., Mohammed, M. A., ... Aveyard, P. (2015). Lack of attentional retraining effects in cigarette smokers attempting cessation: A proof of concept double-blind randomised controlled trial. Drug and Alcohol Dependence, 149, 158-165.

Caponnetto, P., \& Polosa, R. (2008). Common predictors of smoking cessation in clinical practice. Respiratory Medicine, 102(8), 1182-92.

Carpenter, M. J., Saladin, M. E., Larowe, S. D., McClure, E. A., Simonian, S., Upadhyaya, H. P., \& Gray, K. M. (2014). Craving, cue reactivity, and stimulus control among early-stage young smokers: effects of smoking intensity and gender. Nicotine \& Tobacco Research, 16(2), 208-15.

Doran, N. (2014). Sex differences in smoking cue reactivity: craving, negative affect, and preference for immediate smoking. The American Journal on Addictions / American Academy of Psychiatrists in Alcoholism and Addictions, 23(3), 211-7.

Fidler, J. A., \& West, R. (2009). Self-perceived smoking motives and their correlates in a general population sample. Nicotine \& Tobacco Research, 11(10), 1182-8.

Field, M., \& Cox, W. M. (2008). Attentional bias in addictive behaviors: a review of its development, causes, and consequences. Drug and Alcohol Dependence, 97(1-2), 120.

Field, M., \& Duka, T. (2004). Cue reactivity in smokers: the effects of perceived cigarette availability and gender. Pharmacology, Biochemistry, and Behavior, 78(3), 647-52.

Field, M., \& Powell, H. (2007). Stress increases attentional bias for alcohol cues in social drinkers who drink to cope. Alcohol and Alcoholism, 42(6), 560-6.

Field, M., \& Quigley, M. (2009). Mild stress increases attentional bias in social drinkers who drink to cope: A replication and extension. Experimental and Clinical Psychopharmacology, 17(5), 312-319.

Franken, I. H. A. (2003). Drug craving and addiction: integrating psychological and neuropsychopharmacological approaches. Progress in Neuropsychopharmacology and Biological Psychiatry, 27(4), 563-79. 
George, O., \& Koob, G. F. (2010). Individual differences in prefrontal cortex function and the transition from drug use to drug dependence. Neuroscience and Biobehavioral Reviews, 35(2), 232-47.

Havermans, A., Vuurman, E. F., Riedel, W. J., \& van Schayck, O. C. P. (2015). How to win -and not only fight- a running battle: Web-based attention bias modification training for active smokers. Submitted.

Heatherton, T. F., Kozlowski, L. T., Frecker, R. C., \& Fagerström, K. O. (1991). The Fagerström Test for Nicotine Dependence: a revision of the Fagerström Tolerance Questionnaire. British Journal of Addiction, 86, 1119-1127.

Hiscock, R., Bauld, L., Amos, A., Fidler, J. A., \& Munafò, M. R. (2012). Socioeconomic status and smoking: a review. Annals of the New York Academy of Sciences, 1248, 107-23.

Hogarth, L. C., Mogg, K., Bradley, B. P., Duka, T., \& Dickinson, A. (2003). Attentional orienting towards smoking-related stimuli. Behavioural Pharmacology, 14, 153-160.

Kerst, W. F., \& Waters, A. J. (2014). Attentional retraining administered in the field reduces smokers' attentional bias and craving. Health Psychology, 33(10), 1232-40.

Littel, M., Euser, A. S., Munafò, M. R., \& Franken, I. H. A. (2012). Electrophysiological indices of biased cognitive processing of substance-related cues: A meta-analysis. Neuroscience and Biobehavioral Reviews, 36(8), 1803-1816.

Lopes, F. M., Pires, A. V., \& Bizarro, L. (2014). Attentional bias modification in smokers trying to quit: a longitudinal study about the effects of number of sessions. Journal of Substance Abuse Treatment, 47(1), 50-7.

Mogg, K., \& Bradley, B. P. (2002). Selective processing of smoking-related cues in smokers: manipulation of deprivation level and comparison of three measures of processing bias. Journal of Psychopharmacology, 16(4), 385-392.

Mogg, K., Field, M., \& Bradley, B. P. (2005). Attentional and approach biases for smoking cues in smokers: An investigation of competing theoretical views of addiction. Psychopharmacology, 180, 333-341.

Pennanen, M., Broms, U., Korhonen, T., Haukkala, A., Partonen, T., Tuulio-Henriksson, A., ... Kaprio, J. (2014). Smoking, nicotine dependence and nicotine intake by socioeconomic status and marital status. Addictive Behaviors, 39(7), 1145-51.

Perlato, A., Santandrea, E., Della Libera, C., \& Chelazzi, L. (2014). Biases of attention in 
chronic smokers: Men and women are not alike. Cognitive, Affective, \& Behavioral Neuroscience, 14(2), 742-755.

Peuker, A. C., \& Bizarro, L. (2013). Attentional avoidance of smoking cues in former smokers. Journal of Substance Abuse Treatment.

Pogun, S., \& Yararbas, G. (2009). Sex differences in nicotine action. Handbook of Experimental Pharmacology, (192), 261-91.

Siahpush, M., McNeill, A., Borland, R., \& Fong, G. T. (2006). Socioeconomic variations in nicotine dependence, self-efficacy, and intention to quit across four countries: findings from the International Tobacco Control (ITC) Four Country Survey. Tobacco Control, 15 Suppl 3(suppl_3), iii71-5.

Van Breukelen, G. J. P. (2006). ANCOVA versus change from baseline: more power in randomized studies, more bias in nonrandomized studies [corrected]. Journal of Clinical Epidemiology, 59(9), 920-5.

Vollstädt-Klein, S., Loeber, S., Winter, S., Leménager, T., von der Goltz, C., Dinter, C., ... Kiefer, F. (2011). Attention shift towards smoking cues relates to severity of dependence, smoking behavior and breath carbon monoxide. European Addiction Research, 17(4), 217-24.

Waters, A. J., Shiffman, S., Sayette, M. A., Paty, J. A., Gwaltney, C. J., \& Balabanis, M. H. (2003). Attentional bias predicts outcome in smoking cessation. Health Psychology, 22(4), 378-87.

Zack, M., Belsito, L., Scher, R., Eissenberg, T., \& Corrigall, W. A. (2001). Effects of abstinence and smoking on information processing in adolescent smokers. Psychopharmacology, 153, 249-257.

Zhou, X., Nonnemaker, J., Sherrill, B., Gilsenan, A. W., Coste, F., \& West, R. (2009). Attempts to quit smoking and relapse: factors associated with success or failure from the ATTEMPT cohort study. Addictive Behaviors, 34(4), 365-73. 


\section{GENERAL DISCUSSION}


The aim of this thesis project was to provide new insights in neurocognitive mechanisms underlying smoking dependence and possible treatments for smoking cessation. Despite its harmful effects, numerous people are still addicted to smoking. Many smokers are aware of the negative consequences of smoking and want to quit, but have difficulty doing so. Even though there are various pharmacological and behavioral interventions available for smoking cessation, only a small percentage of smokers manages to remain abstinent for longer than one year (Fiore et al., 2008; Rigotti, 2013). Therefore, there is a clear and urgent need for new treatment options. More knowledge about specific neural and cognitive mechanisms in smoking addiction may help in the development of such treatments.

\section{Effects of vaccination against nicotine}

The main underlying factor in smoking addiction is nicotine. Nicotine influences various neural processes that contribute to addiction, such as the reinforcing release of dopamine and consequential behavioral conditioning, and the diminishing balance between motivational and controlling brain regions. Therefore a straightforward step towards prolonged smoking cessation would be reducing or eliminating the amount of nicotine reaching the brain. This can be achieved by means of immunotherapy. However, as nicotine asserts its influence widely over the brain, cutting it off may affect cognitive or behavioral functions. In our first study we used neuroimaging and behavioral paradigms to investigate the effects of nicotine vaccination during cue exposure and a working memory task. The first aim in this study was to prove the concept of this vaccine by showing differences in brain responses between treated and untreated participants after administration of nicotine. In addition we aimed to investigate the effects of reduced nicotine availability on cue reactivity and working memory.

We did not find any effects of vaccination on brain responses to smoking cues or during working memory performance. In addition, we also did not see any effect of acute administration of nicotine before scanning. We did find overall increased responses to smoking cues in bilateral occipital gyrus. Moreover, in all groups brain activity increased with working memory load in several frontal and parietal areas. These results indicate that the vaccine was not effective in reducing nicotine supply to the brain in such an extent that it could influence BOLD responses during our task paradigms. Weekly cigarette intake of immunized participants also did not change after treatment, which confirms the ineffectiveness of this vaccine. Additionally, a parallel randomized controlled trial showed poor clinical efficacy of the same vaccine (Hoogsteder, Kotz, van Spiegel, Viechtbauer, \& 
van Schayck, 2014). In conclusion, the vaccine that we tested (i.e. NicVax) is not likely to be an effective treatment for smoking cessation.

Several other nicotine vaccines are currently under investigation, however none of them has (yet) been proven clinically successful (Hartmann-Boyce, Cahill, Hatsukami, \& Cornuz, 2012). There are various thinkable explanations for the lack of effects in immunotherapy for smokers. First of all, it is possible that in response to the vaccines not enough antibodies are produced to capture all free nicotine molecules in the blood. Also the antibodies produced may not be sufficiently capable to bind nicotine. These two explanations are not mutually exclusive, so both can be effective at the same time. In both cases a certain amount of nicotine will still be able to reach the brain and assert its addicting effects. In support of these hypotheses, three studies showed significant higher abstinence rates in participants with higher concentrations of nicotine antibodies (Cornuz et al., 2008; Hatsukami et al., 2011; Hoogsteder et al., 2014). This indicates that vaccines capable of inducing higher levels of (higher quality) antibodies may be more effective. However, in one of these studies the difference in abstinence between treatment and control groups declined during the time that antibody levels peaked (Cornuz et al., 2008), which contradicts our previous hypothesis. A Single Photon Emission Computed Tomography (SPECT) study by Esterlis and colleagues showed that treatment with the nicotine vaccine NicVax led to a $12.5 \%$ reduction in nicotine binding to receptors in the brain. Unlike other studies using the same vaccine (Hatsukami et al., 2011; Havermans, Vuurman, van den Hurk, Hoogsteder, \& van Schayck, 2014; Hoogsteder et al., 2014), the author found reduced daily cigarette consumption and desire to smoke after vaccination. So it seems that NicVax is successful in reducing the neural level of nicotine to a certain amount, but it is unclear how low these levels need to be to affect smoking behavior. In fact, it is unclear whether eliminating nicotine from the brain would be effective at all, since there are other mechanisms that sustain smoking addiction as well.

This leads us to a second explanation for the overall ineffectiveness of nicotine vaccines. That is, the contribution of nicotine to the complex pathology of addiction may be smaller than expected and therefore reducing or eliminating it from the brain may not be sufficient to breach the addictive cycle. Indeed, studies have shown that other components in tobacco smoke besides nicotine can enhance the reinforcing effects of smoking and thereby are likely to play a role in the maintenance of addiction (Lewis, Miller, \& Lea, 2007; Rose, Salley, Behm, Bates, \& Westman, 2010). Also, specific cues in smokers' environments become associated with anticipation of reward through a process of conditioning (Benowitz, 2010). Over time, these smoking-related cues become highly salient and trigger craving. Therefore, it is possible that after prolonged smoking these 
smoking cues are sufficient to generate rewarding responses in the brain and maintain addiction without the presence of nicotine. Moreover, besides the mesolimbic and prefrontal cortical structures involved in reward and (lack of) cognitive control, other brain areas may be involved in smoking addiction as well. For instance, recent findings indicate a crucial role for the insula in smoking addiction, as damage to the insula was followed by a 'disruption of smoking addiction' (Naqvi \& Bechara, 2009).

Finally, clinical trials have primarily assessed the effects of immunization for smoking cessation, whereas its mechanism of action may be more effective for relapse prevention (Raupach, Hoogsteder, \& van Schayck, 2012). In conclusion, treatment with a nicotine vaccine only does not appear to be a promising tool in smoking cessation for now. However, new vaccines incorporating various more successful strategies to increase nicotine antibody binding capacity are currently in development and may provide better prospects for smoking cessation (Cornish, de Villiers, Pravetoni, \& Pentel, 2013; de Villiers, Cornish, Troska, Pravetoni, \& Pentel, 2013; Keyler, Roiko, Earley, Murtaugh, \& Pentel, 2008; Lockner et al., 2015; Pravetoni et al., 2012). Moreover, future studies should also provide more insights in the optimal way of application for nicotine vaccines. For instance, immunization could become part of a multifaceted interventional approach, also comprising other pharmacological and behavioral methods. In addition, it is important to investigate whether nicotine vaccines could be (more) effective for relapse prevention instead of smoking cessation.

\section{Neural processing of smoking cues}

As mentioned before, various interacting processes contribute to the maintenance of smoking addiction. For instance, an imbalance between brain areas involved in cognitive control and motivation/ reward, and positive and negative reinforcing effects of dopamine have all been implicated to play a role. In addition, conditioned responses to smoking cues form a crucial factor. For long term smokers, such cues will activate the mesolimbic system and thereby trigger craving for cigarettes. Moreover, plastic changes in the brain alter the attentional priority of those stimuli. As a result these cues automatically capture and hold the smokers attention (i.e. attention bias). Disrupting the process of conditioning or the automatic responses to smoking cues may facilitate smoking cessation and prevent relapses. But in order to target these processes it is important to understand their underlying mechanisms. Therefore we have used advanced neuroimaging methods to investigate neural processing of smoking cues under conditions of nicotine deprivation and satiation. In 
chapter 2 we described that our pattern classification algorithm was able to distinguish neural response patterns in object sensitive visual area lateral occipital complex (LOC) to smoking related pictures (cigarettes) from those to visually similar neutral pictures (pencils). Moreover, its ability to discriminate between those two stimulus classes ceased when participants were satiated. From this, we conclude that the effect of abstinence on behaviour can at least be traced back to a basic level of visual object processing. Moreover, this indicates that the mechanism by which a smoker's attention is biased towards smoking cues potentially affects processing in the early visual system.

In order to find out whether other brain regions are also sensitive to deprivation induced differences in salience of smoking related stimuli, we applied a whole brain searchlight analyses (described in chapter 3) based on the same classification paradigm. With this approach, we showed better decoding of neural response patterns to smoking related (cigarettes) vs neutral (pencils) cues in bilateral lateral occipital areas. These findings support the results of our previous study as well as our hypotheses on the effect of smoking deprivation on biased processing of smoking related stimuli.

\section{Neural mechanisms in attention bias}

When a visual stimulus is presented, the processing pathway typically proceeds from the primary visual cortex to extra striate and higher-order visual association areas. From there, projections go to multimodal processing regions including the amygdala and prefrontal cortex (Tanaka, 1992). The amygdala, together with other areas of the mesolimbic system (e.g. hippocampus, nucleus accumbens, and ventral tegmental area) is involved in processing the emotional or motivational value of stimuli. Smoking related cues activate these areas more than neutral cues, inducing a state of 'wanting' and indicating high motivational value probably related to the possibility of future reward (e.g. Hester \& Luijten, 2013). In addition, reciprocal connections between insula and amygdala are involved in this process and may be responsible for the conscious feeling of craving elicited in response to smoking related cues (Naqvi \& Bechara, 2009).

Meanwhile, reciprocal connections of the amygdala to various levels of the ventral-stream are thought to enhance sensory processing of emotionally salient stimuli in the visual cortex (Amaral, Behniea, \& Kelly, 2003). In addition, the amygdala may modulate activity within visual processing areas via its projections to frontal sites that control the allocation of attentional resources (Amaral et al., 2003). So the initial patterns of activation over occipital and temporal cortex would be equal for smoking related and 
neutral cues. However, slightly later, after feedback from other structures such as the amygdala reaches these areas, processing would be aimed more selectively at the smoking related cues. In line with this we demonstrated significantly distinct responses in object sensitive visual area LOC to smoking related and neutral cues, when their emotional valence (i.e. saliency) differed maximally, i.e. when participants were deprived of smoking.

As a final step in the process, a cortical system involved in cognitive control becomes activated. In particular, the anterior cingulate cortex (ACC) modulates emotional and motivational processing and exerts top-down control on the mesolimbic system. The ACC is also thought to be part of the brain's attentional networks and has been associated with conflict monitoring in the presence of competing response alternatives (Kerns et al., 2004). Therefore ACC may detect the presence of competition for processing resources when smokers are presented with both smoking related and neutral cues. Subsequently, other prefrontal cortical areas may increase top-down attentional control aimed at maintaining goal-directed processing. However, since smokers are typically characterized by an ineffective cognitive control system, mesolimbic activation in response to the motivational value of smoking cues may be overpowering, resulting in biased processing. In line with this, it was suggested that attention bias could be avoided by preparatory upregulation of prefrontal control systems to focus on goal-related stimuli, instead of distracting salient stimuli (Hester \& Luijten, 2013; Kerns et al., 2004).

\section{Attention bias modification for smokers}

Neural responses to smoking related cues are automatic and happen outside of the smokers' voluntary control. They also often lead to increased attentional allocation and engagement on smoking cues. This attention bias can lead to increased craving for cigarettes and has been implicated in relapse. Therefore, reducing attention bias could contribute to smoking cessation and the prevention of relapses. In order to target this enhanced automatic attentional processing, implicit training paradigms have been developed and applied. In chapter 4 we described a randomized controlled trial offering five sessions of online attention bias modification ( $\mathrm{ABM}$ ) training for smokers. We found that attention bias for smoking cues was significantly attenuated by these five semi-daily sessions of ABM training. Moreover, the effect of our training was both robust and generalizable as it maintained after a week without training and was also present when novel cues were used in the assessment of attention bias. 
However, we did not find a significant effect of training on our assessment of general subjective craving. This type of craving that smokers experience irrespective of situational cues is often referred to as tonic, or background craving (Dunbar, Shiffman, Kirchner, Tindle, \& Scholl, 2014; Ferguson \& Shiffman, 2009). Background craving levels can fluctuate during a day or over days, and are higher in more dependent smokers (Dunbar et al., 2014). Cue-elicited craving on the other hand, is phasic craving that arises quickly, in response to situational cues and is totally independent of background craving and smoking dependence (Dunbar et al., 2014). Accordingly, we believe it is likely that ABM trainings will not affect background craving, but only craving in response to smoking cues. As a consequence of the training less attentional processing resources will be directed at the smoking related content and therefore smokers may experience less cue-elicited craving. In line with this Kerst and Waters showed that multiple sessions of ABM training significantly reduced cued- but not uncued craving (Kerst \& Waters, 2014). So even though ABM did not decrease background craving in our study, its capacity to diminish attention bias is still very promising. If a decreased attention bias indeed reduces craving in response to smoking cues, this may be an important mechanism in relapse prevention. Future studies should further investigate this relation between $\mathrm{ABM}$ and cue-elicited craving in relapse prevention.

As strength of attention bias varies between smokers, it is also conceivable that ABM will not work equally well for all smokers. Therefore we have investigated the effects of several characteristics of smokers on attention bias and its malleability (chapter 5). We did not find any features affecting attention bias at baseline, which is surprising, as previous studies have shown attention bias differs with gender, degree of tobacco dependence and age (Attwood, O’Sullivan, Leonards, Mackintosh, \& Munafò, 2008; Hogarth, Mogg, Bradley, Duka, \& Dickinson, 2003; Littel, Euser, Munafò, \& Franken, 2012; Mogg \& Bradley, 2002; Mogg, Field, \& Bradley, 2005; Perlato, Santandrea, Della Libera, \& Chelazzi, 2014; Vollstädt-Klein et al., 2011; Zack, Belsito, Scher, Eissenberg, \& Corrigall, 2001). Moreover, the only significant predictor of the training effect was training type, which is a very obvious finding since we had already demonstrated that the ABM training was more effective in reducing attention bias than the control training. The lack of significant predictors of $\mathrm{ABM}$ effects besides the one of training, suggests that ABM may be equally effective for all groups of smokers. This is a very encouraging finding for the feasibility of clinical implementation of ABM trainings for smokers.

Together with two previous studies (Kerst \& Waters, 2014; Lopes, Pires, \& Bizarro, 2014), our study provides strong evidence that at least three sessions of ABM training significantly and robustly reduce attention bias to smoking cues. However it is 
unclear how these findings should be interpreted in terms of clinical relevance. All three studies did not find an effect of ABM on background craving, but Kerst and Waters did report decreased craving in response to smoking cues. The latter finding indicates a possible mechanism by which ABM could promote abstinence in smokers. Besides, one recent study showed that $\mathrm{ABM}$ training was significantly more effective than a control training in fostering continued abstinence among heavy smokers (> 15 cigarettes/day) (Elfeddali, De Vries, \& Wiers, n.d.). Interestingly, our own results also suggest that ABM may be more successful in moderate smokers who smoke $11-20$ cigarettes a day. We did not find a similar interaction for heavier smokers; however this may be a consequence of the smaller sample size of that subgroup.

\section{Mechanism of $A B M$}

Based on the findings of ABM studies, it is possible to speculate on the underlying mechanism by which it influences attention bias and/or smoking behavior. For instance, it seems likely that by repeated practice of this training, smokers develop an automatic attentional avoidance reaction to smoking cues. Several researchers have suggested that ABM specifically affects attentional disengagement, but not initial attentional focus, as early studies in anxiety and alcohol abuse only found effects of ABM on trials with longer stimulus presentation times (i.e. $\geq 500 \mathrm{~ms}$ ) (e.g. 27,28). In line with this, Lopes and colleagues also found that after ABM, attention bias in smokers was lower for longer stimulus presentation times (Lopes et al., 2014). One other study incorporated shorter (200 $\mathrm{ms}$ ) and longer (500 ms) stimulus presentation times in ABM for smokers. Although they found larger baseline attention bias for the longer presented stimuli, there was no effect of stimulus presentation time on the effect of ABM (Field, Duka, Tyler, \& Schoenmakers, 2009). In sum, ABM seems more likely to influence attentional disengagement from smoking related cues, than automatic initial orienting towards them. However, only a small body of evidence supports this hypothesis, and ABM may cause attentional avoidance either way.

Automatic attentional avoidance may interrupt conditioned motivational responses to smoking cues signaling an upcoming nicotinic reward. As a consequence these cues may be devaluated and trigger less strong craving for cigarettes. This is in line with a study showing reduced cue-elicited cigarette craving after ABM (Kerst \& Waters, 2014). Moreover, automatic attentional avoidance will lead to decreased exposure to smoking cues. This will in its turn cause less frequent occurrences of cue-elicited craving. So 
implicitly, ABM may help prolong abstinence by reducing smokers' vulnerability to smoking cues.

Besides ABM's presumed effect on automatic attentional processes, it may also influence conscious attentional control to a certain extent. Particularly when smokers become aware of the contingencies of the training (by repeated practice or through explicit instructions) the latter may be the case. So when smokers realize that their attention is trained (and that this may have consequences for their smoking habits), they will also become more aware of cues in their natural environments that trigger craving. Moreover, they may deliberately try to avoid these cues and/or try to resist or decrease craving by asserting more cognitive control. So in addition to implicitly reducing vulnerability to smoking-related cues, ABM may also (in specific conditions) explicitly increase attentional control. These hypothesized mechanisms are depicted in figure 1.

Altogether ABM likely reduces vulnerability in response to smoking cues, but there is no reason to expect reduced background craving for cigarettes. Hence, it is not surprising that many studies (including ours) failed to find an effect of ABM on questionnaires measuring background craving (Attwood et al., 2008; Field et al., 2009; Kerst \& Waters, 2014; Lopes et al., 2014). In addition, ABM may be especially effective when explicit instructions are provided that reveal the training's contingencies. In that case, augmented cognitive control over craving may be an additional mechanism by which a smoker can extend smoking abstinence. In conclusion, ABM specifically targets automatic responses to smoking cues and thereby may help smokers who want to remain abstinent after a quit attempt. Since it will not directly affect background craving levels or smoking behavior, ABM on its own may not be highly effective for smoking cessation. Instead, due to its specific target it may be more suitable for relapse prevention. Moreover, application of $\mathrm{ABM}$ in addition to other treatments may offer better outcomes. 


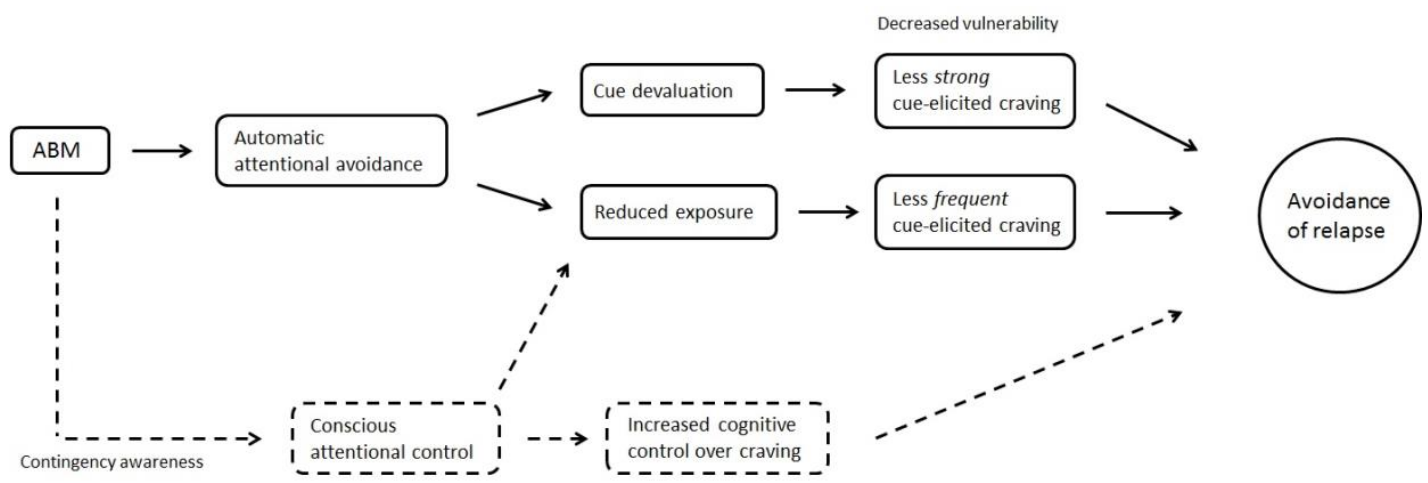

Figure 1. Proposed model of the mechanism of ABM.

\section{Methodological considerations \& future suggestions}

The currently published studies on ABM do provide support for the ABM's ability to reduce attention bias, but show heterogeneous results on clinical outcome measures. The differences in outcomes of these studies are possibly underlain by their dissimilarities in methodology. In order to optimize ABM's effectivity for clinical use, it is important to critically evaluate methodological options and choose the optimal design. In the next section several relevant considerations in the design and methodology of ABM trials will be discussed.

First of all, it is important to determine whether the changes in dependent variables after training are truly caused by the training itself and are not a consequence of other factors changing with time. For instance, in ABM research it is generally assumed that attention bias is a trait-like construct that remains stable over time; however it could also be possible that it fluctuates due to changing contextual factors. If that is the case, differences in attention bias between baseline and post-test would merely be a consequence of its unstable character. Moreover, changes in attention bias could also occur due to priming effects of the task. So, it is possible that the systemic exposure to smoking-related cues in the trainings may influence attention bias. In addition, simply being involved in a research trial related to smoking could make smokers more aware of their smoking habits in a way that also affects their attention bias. Altogether, there are various thinkable factors that could influence attention bias instead of or in addition to the training. 
In order to control for these various effects, it is important to include a control group who participate under the same circumstances but whose attention is not actively trained away from smoking cues. Some early studies used a control group that received a training opposite to the real intervention, i.e. training attention towards smoking related images (Attwood et al., 2008; Field et al., 2009). However this complicated interpretation of their findings. If there was, for instance an increase in craving in the participants trained to attend smoking cues, it would be impossible to infer whether this is an effect of the 'attend' training or a more natural occurring increase that is prevented in the 'avoid' training group. Therefore a better (and most usually applied) option is to add a control group whose attention is not trained in any direction. This is usually achieved by applying the same dotprobe that is used to measure attention bias, during which the dot appears following a smoking related picture in half of the trials (Begh et al., 2015; Field et al., 2009; Kerst \& Waters, 2014; Lopes et al., 2014; McHugh, Murray, Hearon, Calkins, \& Otto, 2010). This type of control training was also implemented in our own ABM trial. However, some researchers have suggested that a 50/50 dot-probe task may still influence attention bias towards drug-related cues (Schoenmakers et al., 2010). Since participants will try to become faster in all trials, the difference in response latencies to both cues, i.e. our measure of attention bias, may diminish. Therefore an alternative option for a control condition that shows the same stimuli as the ABM training without influencing attention bias, could be a categorization or implicit association task (Schoenmakers et al., 2010). In such a task participants classify a target stimulus to a specific condition (e.g. smoking or non-smoking related) by pressing designated buttons for each condition.

Another important consideration for the interpretation of the effects of $A B M$ is the role of task specific learning effects. In ABM studies, the training is usually based on the task which is used as dependent measure. Therefore it is possible that during performance of the post-test, participants may remember specific stimulus pairs and the associated location of the dot-probe from the previous sessions and are therefore able to respond much quicker. In order to demonstrate that the training effectively changed implicit attentional processes, some of the stimuli used in the pre- and post-test should not be used in the trainings. In that way attention bias is measured with stimuli that participants have not seen before. Single session ABM studies did not find an effect of training on attention bias when using untrained stimuli (Field et al., 2009; McHugh et al., 2010). However this lack of effects may be due to the fact that only a single session of ABM was applied. In general, studies using multiple sessions have been shown more successful in reducing attention bias (eg. 17,26). In line with this, two studies (including our study) that used multiple sessions of $\mathrm{ABM}$ training found training effects when novel cues were used in the assessment 
(Lopes et al., 2014). A third multiple-session ABM study did not find any effects of the training using untrained stimuli, but these findings may be biased by the lack of attention bias at baseline.

Besides counteracting training effects, the use of a broad range of (trained and) untrained stimuli is also important to assure the generalizability of findings in ABM studies. Clearly, ABM will only be clinically relevant when it will not just reduce attention bias to the specific cues in the task, but also to the cues that smokers encounter during their daily lives. Increasing the range and variety of stimuli used in the task will augment the likelihood that effects on attention bias will transfer to smokers' natural environment. A uniform set of smoking related stimuli may however, not relate to all smokers, since their habits and preferences vary. Therefore, the ecological validity of the training, and thereby its clinical relevance, could further be improved by using individual stimuli adapted to each participant's smoking-environment (Fadardi, Cox, \& Klinger, 2006). Accordingly, personal smoking related pictures provoked a larger increase in craving for cigarettes than standard smoking related pictures (Conklin et al., 2015). Nevertheless the use of individualized stimuli is time-consuming and more complicated to implement. However, with the newest techniques making ABM easily accessible for individuals (for instance on smartphones), developers could possibly allow smokers to upload their own pictures in the future.

Besides the possibility of using individualized stimuli, application of ABM via smartphones or other personal devices may hold other benefits. For instance, this way ABM can be used more frequently, which could increase its effects. Not only by more practice and exposure, but also because training seems to be more effective when multiple sessions are spaced apart than when they are massed together (e.g. 34). Furthermore, a recent review on the relevance of $\mathrm{ABM}$ in addiction argues that $\mathrm{ABM}$ may be only effective when applied in the participants' naturalistic environments, where they have used substances and encountered cues (Christiansen, Schoenmakers, \& Field, 2015). This suggestion is supported by the findings of Kerst and Waters (Kerst \& Waters, 2014), who provided ABM training on mobile devices that participants carried with them during their normal daily activities. Nevertheless further research is necessary to draw definite conclusions on the effectivity of ABM in smokers' home environments. Secondly, motivating participants to improve training performance and control over their attention may be beneficial since it will promote compliance and active participation. Practically, participants' motivation could be increased by implementing $\mathrm{ABM}$ as a game in which they can keep a score and compete with others (i.e. gamification). 
Another central aspect in such motivation-enhancing strategies is contingency awareness. When participants become aware of the experimental contingencies they may consciously try to adjust their performance to what they think the researchers expect. For instance, in an ABM trial, when participants realize they are trained away from smoking related cues, they may think that the researchers aim to decrease their level of craving or smoking behavior. In subsequent behavioral measures, participants may understate their craving levels or cigarette consumption. The effects of contingency awareness have not been studied on $\mathrm{ABM}$ for smokers, but two studies have investigates these effects on $\mathrm{ABM}$ for heavy drinkers (Field \& Eastwood, 2005; Field et al., 2007). One of them did not find effects of awareness (Field \& Eastwood, 2005), whereas the other showed that ABM decreased background craving for alcohol, only for participants who had been aware of the experimental contingencies (Field et al., 2007). Consequently, the question is whether their craving outcome was falsely influenced by participants providing (socially) desirable answers, or whether contingency awareness may actually be beneficial for ABM. In line with the latter hypothesis, it is thinkable that awareness of the underlying attentional processes fueling their addiction could help smokers gain more control over their smoking habits. Besides, informing participants about the experimental contingencies may be inevitable when ABM will be applied as a clinical tool.

An important limitation to $\mathrm{ABM}$ research is the low reliability of the dot-probe task as a measure of attention bias (Ataya et al., 2012; Price et al., 2015). This is specifically a concern for studies that relate individual differences in attention bias to relapse potential (e.g.39), since these differences could just be a consequence of the weak stability of the dot-probe task. However, even if the dot-probe task is an unreliable outcome measure for ABM's effect on attention bias, trainings based on the dot-probe task may still be effective in reducing clinical outcomes like (cue-induced) craving or smoking behavior. Nevertheless, future studies should be critical in their design and methodology when using the dot-probe task. Several factors can be taken into account in order to increase reliability.

First of all, it is likely that reaction time measures related to cognitive processes are limited in their reliability since they can be influenced by many irrelevant factors (e.g. response selection latency, general processing speed, equipment). One way to avoid these sources of error is by using measures other than reaction times, such as eye tracking. The reliability of eye tracking indices of attention bias have not (yet) been investigated, however one study showed that they correlated with reaction time measures, suggesting convergent validity (Mogg, Garner, \& Bradley, 2007). Although eye tracking may be a more stable measure of attention bias it is clearly not applicable for the training part of ABM. For assessment of attention bias it has also been suggested that a modified stroop 
task is more reliable than the dot-probe (Ataya et al., 2012), however also this task is not applicable for ABM training. Including a large number of trials in the dot-probe, may also increase its reliability (Ataya et al., 2012; Price et al., 2015). In line with this, it is recommended to rescale outliers instead of removing them from the dataset (Price et al., 2015). Furthermore, researchers should be critical on the way they calculate attention bias and take into account within-subject and within-session variability (Price et al., 2015). Finally, it is important to note that reliability and validity are not synonymous, so a less reliable measure like the dot probe could still be valid to quantify or train attention bias.

\section{Conclusion and clinical implications}

In this thesis several studies are described that investigated neural processing of smoking cues and the mechanisms underlying two specific methods for smoking cessation or relapse prevention. Based on the findings from our first study we conclude that even though the vaccine we used was ineffective, new nicotine vaccines may prove useful in the future for reducing nicotine levels in the brain. As nicotine dependence is usually regarded as the major determinant of persistent smoking (Shadel, Shiffman, Niaura, Nichter, \& Abrams, 2000), this may sound promising. However, we argue that other mechanisms may be just as influential in maintaining addiction to cigarettes. For instance, conditioned responses to situational smoking related cues may play an important role. Accordingly, smokers are known to develop an attention bias towards these craving-triggering cues. We found that selective processing of smoking related cues under influence of smoking abstinence (i.e. increased salience) can be traced back to the object sensitive visual area LOC. This finding may provide a new target for smoking cessation interventions; for instance non-invasive brain stimulation techniques may be used to manipulate brain activity in LOC.

Various brain regions in visual, motivational and attentional systems are thought to be involved in the processing of smoking related cues and the consequential attention bias. In light of our findings, future studies may investigate which specific regions send input to LOC, for instance by means of a seed-based connectivity analysis. It would also be very interesting for future research to find out whether and how disruption of processing in each of these systems (for instance by means of non-invasive brain stimulation techniques) influences attention bias and craving in smokers. Moreover, some brain areas (e.g. ACC) are specifically thought to play a role in signaling or biasing competition for processing resources. However, since participants in our fMRI study were presented with one image at a time, there was no direct competition for attentional resources between smoking related 
and neutral cues. Therefore it would be interesting for future studies to replicate our research with a stimulus presentation paradigm that allows for attentional competition, like a dot probe task. Additionally, multivariate searchlight results can be improved by using cortex based alignment to increase spatial correspondence across individual brains.

We have also demonstrated that training based on an ABM paradigm may reduce attention bias and thereby decrease smokers' exposure to, and vulnerability for, smoking cues. In the end this may help to avoid relapses in smokers who have made a quit attempt. Besides, as ABM does not seem to influence background craving, it may not be a suitable tool for smoking cessation. In addition we found that $\mathrm{ABM}$ may be effective for all smokers regardless of personal characteristics or smoking behavior. This will make future clinical applications less complicated. Moreover, ABM is thought to be especially effective when tailored to individual smokers and applied in their natural environments. Effectivity may be further increased by critically evaluating its methodology and choosing an optimal design. In addition, specific pharmacological substances or brain stimulation may enhance ABM training. Specifically, transcranial direct current stimulation (tDCS) may temporarily disrupt prefrontal attentional responses to salient stimuli (Boggio et al., 2009; R.W. Wiers, Gladwin, Hofmann, Salemink, \& Ridderinkhof, 2013). This could help participants perform better at the training, or directly influence attention bias through plasticity changes in the brain.

Overall, both nicotine vaccines and ABM trainings could be helpful for smokers who want to remain abstinent. However, as smoking addiction is a complex process that involves interactions between various neural and cognitive processes, it is likely that an intervention for smoking cessation (or relapse prevention) will only be successful when it targets a combination of these aspects. For instance, ABM could be applied combined with nicotine replacement therapies (NRT) and/or behavioral therapies. In this way, NRT may reduce smokers' increased background craving after a quit attempt, and help them getting used to gradually decreasing nicotine supply. Nicotine vaccines may be applicable in a similar way. In addition ABM may reduce smokers' sensitivity to craving-triggering cues and, in case of contingency awareness, make them more aware of these craving triggers. Behavioral therapies and counseling sessions may create awareness of smoking habits in a similar way, and in addition may provide specific strategies to deal with cravings or change these habits. Besides ABM, future studies should also investigate the option to target different levels of neural processing with brain stimulation techniques in order to decrease attention bias and/or craving in response to smoking cues. In sum, the combined use of various therapeutic options may call an end to the worldwide smoking epidemic and finally show that we have progressed from the time of the cowboys. 


\section{References}

Amaral, D. G., Behniea, H., \& Kelly, J. L. (2003). Topographic organization of projections from the amygdala to the visual cortex in the macaque monkey. Neuroscience, 118(4), 1099-1120.

Ataya, A. F., Adams, S., Mullings, E., Cooper, R. M., Attwood, A. S., \& Munafò, M. R. (2012). Internal reliability of measures of substance-related cognitive bias. Drug and Alcohol Dependence, 121(1-2), 148-151.

Attwood, A. S., O'Sullivan, H., Leonards, U., Mackintosh, B., \& Munafò, M. R. (2008). Attentional bias training and cue reactivity in cigarette smokers. Addiction, 103(11), $1875-82$.

Begh, R., Munafò, M. R., Shiffman, S., Ferguson, S. G., Nichols, L., Mohammed, M. A., ... Aveyard, P. (2015). Lack of attentional retraining effects in cigarette smokers attempting cessation: A proof of concept double-blind randomised controlled trial. Drug and Alcohol Dependence, 149, 158-165.

Benowitz, N. L. (2010). Nicotine addiction. The New England Journal of Medicine, 362, 2295-303.

Boggio, P. S., Liguori, P., Sultani, N., Rezende, L., Fecteau, S., \& Fregni, F. (2009). Cumulative priming effects of cortical stimulation on smoking cue-induced craving. Neuroscience Letters, 463(1), 82-6.

Cepeda, N. J., Pashler, H., Vul, E., Wixted, J. T., \& Rohrer, D. (2006). Distributed practice in verbal recall tasks: A review and quantitative synthesis. Psychological Bulletin, 132(3), 354-80.

Christiansen, P., Schoenmakers, T. M., \& Field, M. (2015). Less than meets the eye: Reappraising the clinical relevance of attentional bias in addiction. Addictive Behaviors, 44, 43-50.

Conklin, C. A., Vella, E. J., Joyce, C. J., Salkeld, R. P., Perkins, K. A., \& Parzynski, C. S. (2015). Examining the relationship between cue-induced craving and actual smoking. Experimental and Clinical Psychopharmacology, 23(2), 90-96.

Cornish, K. E., de Villiers, S. H. L., Pravetoni, M., \& Pentel, P. R. (2013). Immunogenicity of individual vaccine components in a bivalent nicotine vaccine differ according to vaccine formulation and administration conditions. PloS One, 8(12), e82557. 
Cornuz, J., Zwahlen, S., Jungi, W. F., Osterwalder, J., Klingler, K., van Melle, G., ... Cerny, T. (2008). A vaccine against nicotine for smoking cessation: a randomized controlled trial. PloS One, 3(6), e2547.

de Villiers, S. H. L., Cornish, K. E., Troska, A. J., Pravetoni, M., \& Pentel, P. R. (2013). Increased efficacy of a trivalent nicotine vaccine compared to a dose-matched monovalent vaccine when formulated with alum. Vaccine, 31(52), 6185-93.

Dunbar, M. S., Shiffman, S., Kirchner, T. R., Tindle, H. A., \& Scholl, S. M. (2014). Nicotine dependence, "background" and cue-induced craving and smoking in the laboratory. Drug and Alcohol Dependence, 142, 197-203.

Elfeddali, I., De Vries, H., \& Wiers, R. W. (n.d.). Preventing short-term smoking relapse among smokers via a Web-based multiple-session attentional bias modification training: a randomized controlled trial.

Fadardi, J. S., Cox, W. M., \& Klinger, E. (2006). Individualized versus General Measures of Addiction-Related Implicit Cognitions. In R. W. Wiers \& A. W. Stacy (Eds.), Handbook of Implicit Cognition and Addiction (pp. 121-133). Thousand Oaks, CA: Sage.

Ferguson, S. G., \& Shiffman, S. (2009). The relevance and treatment of cue-induced cravings in tobacco dependence. Journal of Substance Abuse Treatment, 36(3), 235243.

Field, M., Duka, T., Eastwood, B., Child, R., Santarcangelo, M., \& Gayton, M. (2007). Experimental manipulation of attentional biases in heavy drinkers: do the effects generalise? Psychopharmacology, 192(4), 593-608.

Field, M., Duka, T., Tyler, E., \& Schoenmakers, T. M. (2009). Attentional bias modification in tobacco smokers. Nicotine \& Tobacco Research, 11(7), 812-22.

Field, M., \& Eastwood, B. (2005). Experimental manipulation of attentional bias increases the motivation to drink alcohol. Psychopharmacology, 183(3), 350-7.

Fiore, M. C., Jaén, C. R., Baker, T. B., Bailey, W. C., Benowitz, N. L., Curry, S. J., ... Wewers, M. E. (2008). Treating tobacco use and dependence: 2008 update. Rockville(MD): US Department of Health and Human Services 2008.

Hartmann-Boyce, J., Cahill, K., Hatsukami, D., \& Cornuz, J. (2012). Nicotine vaccines for smoking cessation. The Cochrane Database of Systematic Reviews, 8, CD007072.

Hatsukami, D. K., Jorenby, D. E., Gonzales, D., Rigotti, N. A., Glover, E. D., Oncken, C. 
A., ... Rennard, S. I. (2011). Immunogenicity and smoking-cessation outcomes for a novel nicotine immunotherapeutic. Clinical Pharmacology and Therapeutics, 89(3), 392-9.

Havermans, A., Vuurman, E. F. P. M., van den Hurk, J., Hoogsteder, P., \& van Schayck, O. C. P. (2014). Treatment with a nicotine vaccine does not lead to changes in brain activity during smoking cue exposure or a working memory task. Addiction, 109(8), $1260-7$.

Hester, R., \& Luijten, M. (2013). Neural correlates of attentional bias in addiction. CNS Spectrums, (August), 1-8.

Hogarth, L. C., Mogg, K., Bradley, B. P., Duka, T., \& Dickinson, A. (2003). Attentional orienting towards smoking-related stimuli. Behavioural Pharmacology, 14, 153-160.

Hoogsteder, P. H. J., Kotz, D., van Spiegel, P. I., Viechtbauer, W., \& van Schayck, O. C. P. (2014). Efficacy of the nicotine vaccine 3'-AmNic-rEPA (NicVAX) co-administered with varenicline and counselling for smoking cessation: a randomized placebocontrolled trial. Addiction, 109(8), 1252-9.

Kerns, J. G., Cohen, J. D., MacDonald, A. W., Cho, R. Y., Stenger, V. A., \& Carter, C. S. (2004). Anterior cingulate conflict monitoring and adjustments in control. Science, 303(5660), 1023-6.

Kerst, W. F., \& Waters, A. J. (2014). Attentional retraining administered in the field reduces smokers' attentional bias and craving. Health Psychology, 33(10), 1232-40.

Keyler, D. E., Roiko, S. A., Earley, C. A., Murtaugh, M. P., \& Pentel, P. R. (2008). Enhanced immunogenicity of a bivalent nicotine vaccine. International Immunopharmacology, 8(11), 1589-94.

Lewis, A., Miller, J. H., \& Lea, R. A. (2007). Monoamine oxidase and tobacco dependence. NeuroToxicology, 28(1), 182-95.

Littel, M., Euser, A. S., Munafò, M. R., \& Franken, I. H. A. (2012). Electrophysiological indices of biased cognitive processing of substance-related cues: A meta-analysis. Neuroscience and Biobehavioral Reviews, 36(8), 1803-1816.

Lockner, J. W., Lively, J. M., Collins, K. C., Vendruscolo, J. C. M., Azar, M. R., \& Janda, K. D. (2015). A conjugate vaccine using enantiopure hapten imparts superior nicotine-binding capacity. Journal of Medicinal Chemistry, 58(2), 1005-11.

Lopes, F. M., Pires, A. V., \& Bizarro, L. (2014). Attentional bias modification in smokers 
trying to quit: a longitudinal study about the effects of number of sessions. Journal of Substance Abuse Treatment, 47(1), 50-7.

MacLeod, C., Rutherford, E., Campbell, L., Ebsworthy, G., \& Holker, L. (2002). Selective attention and emotional vulnerability: assessing the causal basis of their association through the experimental manipulation of attentional bias. Journal of Abnormal Psychology, 111(1), 107-23.

McHugh, R. K., Murray, H. W., Hearon, B. A., Calkins, A. W., \& Otto, M. W. (2010). Attentional bias and craving in smokers: the impact of a single attentional training session. Nicotine \& Tobacco Research, 12(12), 1261-4.

Mogg, K., \& Bradley, B. P. (2002). Selective processing of smoking-related cues in smokers: manipulation of deprivation level and comparison of three measures of processing bias. Journal of Psychopharmacology, 16(4), 385-392.

Mogg, K., Field, M., \& Bradley, B. P. (2005). Attentional and approach biases for smoking cues in smokers: An investigation of competing theoretical views of addiction. Psychopharmacology, 180, 333-341.

Mogg, K., Garner, M., \& Bradley, B. P. (2007). Anxiety and orienting of gaze to angry and fearful faces. Biological Psychiatry, 76(3), 163-9.

Naqvi, N. H., \& Bechara, A. (2009). The hidden island of addiction: the insula. Trends in Neurosciences, 32(November), 56-67.

Perlato, A., Santandrea, E., Della Libera, C., \& Chelazzi, L. (2014). Biases of attention in chronic smokers: Men and women are not alike. Cognitive, Affective, \& Behavioral Neuroscience, 14(2), 742-755.

Pravetoni, M., Keyler, D. E., Pidaparthi, R. R., Carroll, F. I., Runyon, S. P., Murtaugh, M. P., ... Pentel, P. R. (2012). Structurally distinct nicotine immunogens elicit antibodies with non-overlapping specificities. Biochemical Pharmacology, 83(4), 543-50.

Price, R. B., Kuckertz, J. M., Siegle, G. J., Ladouceur, C. D., Silk, J. S., Ryan, N. D., \& Dahl, R. E. (2015). Empirical Recommendations for Improving the Stability of the Dot-Probe Task in Clinical Research. Psychological Assessment, 27(2), 365-376.

Raupach, T., Hoogsteder, P. H. J., \& van Schayck, O. C. P. (2012). Nicotine vaccines to assist with smoking cessation: current status of research. Drugs, 72(4), e1-16.

Rigotti, N. A. (2013). Smoking cessation in patients with respiratory disease: existing treatments and future directions. Lancet. Respiratory Medicine, 1(3), 241-50. 
Rose, J. E., Salley, A., Behm, F. M., Bates, J. E., \& Westman, E. C. (2010). Reinforcing effects of nicotine and non-nicotine components of cigarette smoke. Psychopharmacology, 210(1), 1-12.

Schoenmakers, T. M., de Bruin, M., Lux, I. F. M., Goertz, A. G., Van Kerkhof, D. H. A. T., \& Wiers, R. W. (2010). Clinical effectiveness of attentional bias modification training in abstinent alcoholic patients. Drug and Alcohol Dependence, 109(1-3), 3036.

Shadel, W. G., Shiffman, S., Niaura, R., Nichter, M., \& Abrams, D. B. (2000). Current models of nicotine dependence: what is known and what is needed to advance understanding of tobacco etiology among youth. Drug and Alcohol Dependence, 59, $9-22$.

Tanaka, K. (1992). Inferotemporal cortex and higher visual functions. Current Opinion in Neurobiology, 2, 502-505.

Vollstädt-Klein, S., Loeber, S., Winter, S., Leménager, T., von der Goltz, C., Dinter, C., ... Kiefer, F. (2011). Attention shift towards smoking cues relates to severity of dependence, smoking behavior and breath carbon monoxide. European Addiction Research, 17(4), 217-24.

Waters, A. J., Shiffman, S., Sayette, M. A., Paty, J. A., Gwaltney, C. J., \& Balabanis, M. H. (2003). Attentional bias predicts outcome in smoking cessation. Health Psychology, 22(4), 378-87.

Wiers, R. W., Gladwin, T. E., Hofmann, W., Salemink, E., \& Ridderinkhof, K. R. (2013). Cognitive Bias Modification and Cognitive Control Training in Addiction and Related Psychopathology: Mechanisms, Clinical Perspectives, and Ways Forward. Clinical Psychological Science, 1(2), 192-212.

Zack, M., Belsito, L., Scher, R., Eissenberg, T., \& Corrigall, W. A. (2001). Effects of abstinence and smoking on information processing in adolescent smokers. Psychopharmacology, 153, 249-257. 


\section{SUMMARY}

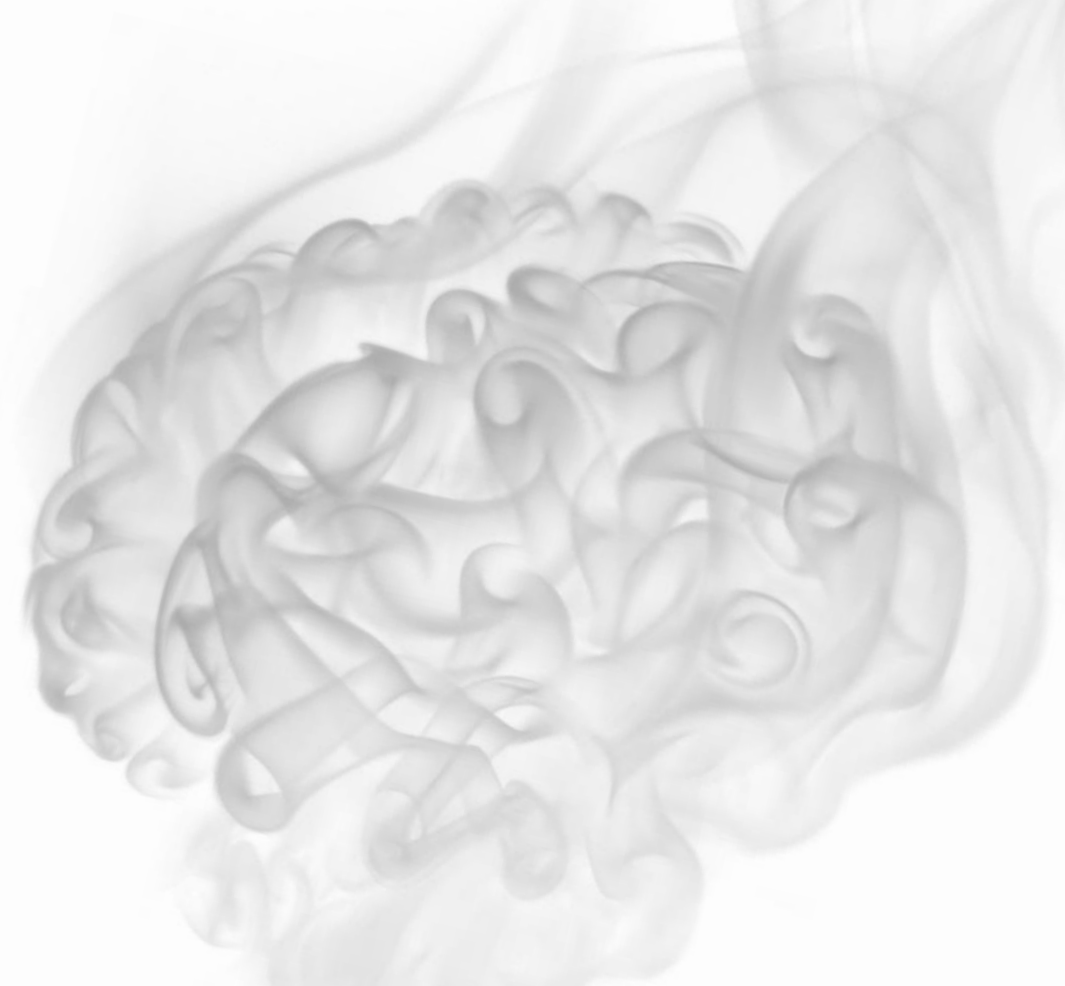


During the 1950's, -60's and -70's, an iconic and highly successful campaign associated smoking cigarettes with being sturdy and masculine, like cowboys. Currently this romanticized image has been scattered by the overwhelming evidence for tobacco's healthdamaging and addictive properties. Still, more than one billion people worldwide smoke tobacco and up to half of them will eventually die as a result of their addiction. Even though many smokers are aware of the detrimental consequences of smoking and want to quit, smoking cessation has not become much easier since the time of the old cowboys. Various pharmacological and behavioral therapies have become available to aid in smoking cessation, but even with the most successful combination of both, one year successful abstinence rates remain well below 50\%. Better treatments are clearly necessary, but in order to get there it is important to understand the mechanisms that make smoking so tremendously addicting.

A major role in addiction to smoking is attributed to the effects of nicotine. In the brain, nicotine stimulates the release of dopamine in the mesolimbic 'reward pathway', which signals the pleasurable experience underlying nicotine's positive reinforcing effects. Longer periods of nicotine exposure induce chronic changes in the brain, which set off the transition to addiction. For instance, an imbalance arises between an ineffective cognitive control system (top-down) based in the prefrontal cortex (PFC) and an overactive motivational system (bottom-up) involving the amygdala and ventral striatum. When the bottom-up incentive signals triggered in the motivational system become very strong, they may overpower top-down cognitive control, causing smokers to not be able to resist lighting up another cigarette. Moreover, the motivational system also becomes activated in response to environmental cues that are paired with the reinforcing outcome of smoking. Over time, these cues can become extremely salient, trigger craving, and smokers may develop an attention bias towards them. This process has also been implicated in the maintenance of smoking behavior.

As addiction to smoking is for a large part a consequence of the actions of nicotine in the brain, one strategy for smoking cessation is to prohibit these actions by precluding nicotine from entering the brain. This is the concept of several nicotine vaccines that are currently in development. The aim of our first study was to demonstrate that immunization attenuates nicotinic stimulation of the brain. In addition we aimed to elucidate its effects on brain and behavioural responses during exposure to smoking cues and a working memory task. Forty-eight male smokers ( $\geq 10$ cigarettes/ day) were randomized to receive five injections with either $400 \mu \mathrm{g} / \mathrm{ml}$ of the 3 'aminomethylnicotine P. aeruginosa r-Exoprotein conjugated vaccine or a placebo over a period of 20 weeks. Subjects were tested on two occasions; once after a nicotine challenge and once after a placebo challenge and were asked to refrain from smoking 10 hours before testing. Reaction times and accuracies were 
recorded during an n-back task. Moreover, regional blood oxygenated level dependent (BOLD) response was measured during this task and during smoking cue exposure. Greater activation was found in response to smoking cues compared to neutral cues in bilateral trans-occipital sulcus; however this effect did not survive correction for multiple comparisons. There was no difference in brain activity to smoking cues between the treatment groups and no effects of acute nicotine challenge were established. For the n-back task we found working memory load-sensitive increases in brain activity in several frontal and parietal areas. However, no effects of immunization or nicotine challenge were observed. In conclusion, we demonstrated that treatment with a nicotine vaccine was not effective in reducing nicotine supply to the brain in such an extent that it influenced neural or behavioral responses during cue exposure or a working memory task. Therefore this vaccine is not likely to be an effective aid in smoking cessation. It is likely that the ineffectivity of this vaccine is caused by a suboptimal antibody response and that other, more potent vaccines could prove to be more effective in the future. Until then we cannot exclude immunotherapy as a possible aid in smoking cessation or relapse prevention.

Besides the direct reinforcing effects of nicotine, conditioned responses to smoking related cues also contribute to the maintenance of smoking addiction. In order to develop a smoking cessation intervention aimed at these responses, it is important to know more about their underlying mechanism. In the study described in chapter 2, we used functional magnetic resonance imaging (fMRI) and multi-voxel pattern analysis (MVPA) to investigate whether tobacco addiction biases basic visual processing in favour of smokingrelated images. We hypothesized that the neural representation of smoking-related stimuli in the lateral occipital complex (LOC) would be elevated after a period of nicotine deprivation compared to a satiated state, but that this would not be the case for object categories unrelated to smoking. Fourteen current smokers ( $\geq 10$ cigarettes a day) were scanned in two sessions: once after 10 hours of nicotine abstinence and once after smoking ad libitum. Regional blood oxygenated level dependent (BOLD) response was measured while participants were presented with 24 blocks of 8 colour-matched pictures of cigarettes, pencils or chairs. The functional data were analysed through a pattern classification approach. In bilateral LOC clusters, the classifier was able to discriminate between patterns of activity elicited by visually similar smoking related (cigarettes) and neutral objects (pencils) above empirically estimated chance levels only when smokers were deprived, but not when they were satiated. For all other stimuli contrasts (cigarettes and chairs, pencils and chairs) there was no difference in discriminability between the deprived and satiated conditions. In conclusion, the discriminability between smoking- and non-smoking visual objects was elevated in object-selective brain region LOC after a period of nicotine abstinence. This indicates that attention bias likely affects basic visual object processing. 
In a consecutive study (chapter 3) we used a multivariate searchlight approach to identify brain regions that are, like LOC, involved in biased processing of smoking related cues under influence of smoking deprivation. Fourteen current smokers $(\geq 10$ cigarettes a day) were scanned in two sessions: once after 10 hours of nicotine abstinence and once after smoking ad libitum. Regional blood oxygenated level dependent (BOLD) response was measured while participants were presented with 24 blocks of 8 colour-matched pictures of cigarettes, pencils or chairs. The functional data were analysed through a pattern classification approach. The multivariate searchlight identified bilateral lateral occipital cortex regions showing better decoding of neural response patterns to visually similar smoking related (cigarettes) and neutral (pencils) images when participants were deprived of smoking. Moreover, the left precentral gyrus showed discriminable responses to smoking related and neutral cues during satiety, but not deprivation. These findings support the results of our previous study, indicating that the mechanism by which a smoker's attention is biased towards smoking cues indeed affects processing in the early visual system.

The automatic activation of the motivation system in response to smoking cues causes craving and alters the attentional priority of those stimuli. These processes are thought to play an important role in the maintenance of smoking addiction and counteracting them may prevent relapse. We aimed to decrease smokers' attention bias towards such cues by means of a web-based attention bias modification (ABM) training (chapter 4). Smokers aged 18-60 years were randomly allocated to ABM $(n=208)$ or a control condition $(n=190)$. Both ABM and control trainings were based on a dot-probe task. Faster reaction times to probes that followed smoking related pictures reflected an attention bias. In the ABM training $80 \%$ of the probes followed neutral pictures. Participants completed five training sessions on (semi-) consecutive days. Attention bias was assessed one day after the final training and after one week follow-up. In the assessment we added a new subset of images to examine whether the effect of the training was generalizable. After five sessions of online training, attention bias was reversed in the participants who had received the intervention training, but not for those in the control training. Moreover this effect was still present at follow-up one week later, and was generalizable to novel smoking related images. Attention bias modification is effective in reducing attention bias to smoking cues and may therefore be of great help in preventing relapses.

Recent studies (including ours) have shown promising effects of Attention Bias Modification (ABM) training to reduce attention bias for smokers. However, the effects of $\mathrm{ABM}$ on craving and smoking behavior are less pronounced. Therefore, we examined whether the effectiveness of this training was influenced by specific characteristics of smokers (chapter 5). Smokers aged 18-60 years were randomly allocated to ABM ( $\mathrm{n}=208)$ or a control condition $(n=190)$. Training was based on a dot-probe task; faster reaction 
times to probes that followed smoking related pictures reflected an attention bias. Participants completed five training sessions on (semi-) consecutive days. Two separate factorial univariate analyses of variance (ANOVA) were applied to examine the effects of the different predictors on attention bias at baseline and post-training. We could not identify any predictors affecting attention bias at baseline. Moreover, no features significantly predicted attention bias after training- except for training type, which indicates that ABM training was more effective than the control condition. These findings show that ABM may be equally beneficial for all smokers, which is very promising for the possible implementation of ABM in clinical practice.

Overall, nicotine vaccines, neural stimulation and ABM trainings could be helpful for smokers who want to remain abstinent. However, as smoking addiction is a complex process that involves interactions between various neural and cognitive processes, it is likely that an intervention for smoking cessation (or relapse prevention) will only be successful when it targets a combination of these aspects. We believe that the combined use of various therapeutic options could call an end to the worldwide smoking epidemic and finally show that we have progressed from the time of the cowboys. 
Neural and cognitive determinants of smoking addiction and cessation 


\section{SAMENVATTING}

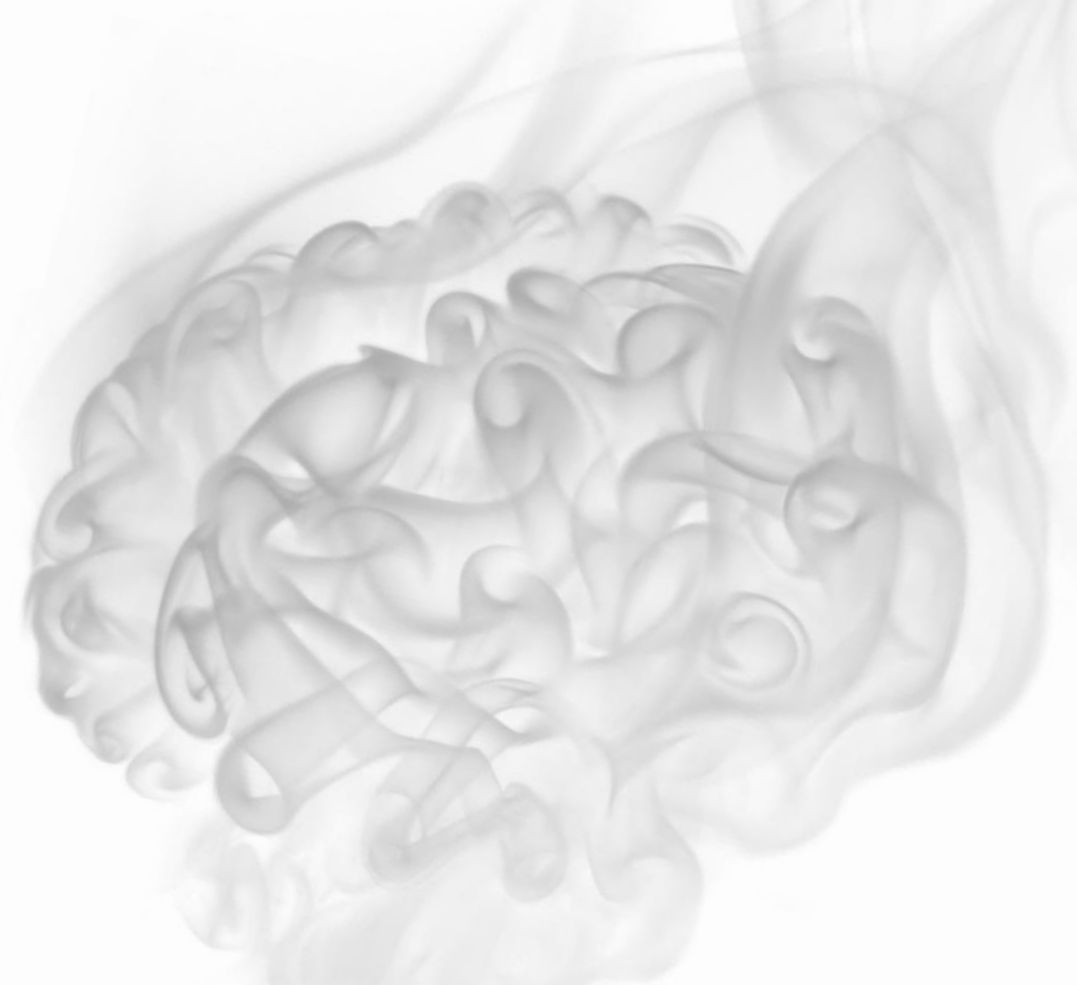


In een iconische en zeer succesvolle reclamecampagne uit de jaren 1950, 1960, en 1970 werd het roken van sigaretten geassocieerd met de stoere en mannelijke karaktertrekken van cowboys. Tegenwoordig is dit geromantiseerde beeld verjaagd door het overweldigende bewijs voor de schadelijke en verslavende eigenschappen van tabak. Toch roken er wereldwijd nog meer dan een miljard mensen, en minstens de helft van hen zal uiteindelijk overlijden aan de gevolgen van hun verslaving. Hoewel veel rokers zich bewust zijn van de negatieve gevolgen van roken en willen stoppen, blijft stoppen met roken nog altijd even moeilijk als in de tijd van de cowboys. Er zijn allerlei farmacologische middelen en gedragstherapieën beschikbaar die kunnen helpen bij het stoppen met roken, maar zelfs bij gebruik van de meest succesvolle combinatie van beiden blijft minder dan de helft van de (ex-)rokers een jaar of langer gestopt. Betere behandelingen zijn dus nodig, maar om zo ver te komen is het belangrijk om de mechanismes te begrijpen die roken zo verschrikkelijk verslavend maken.

Rookverslaving wordt voor een belangrijk deel veroorzaakt en in stand gehouden door nicotine. In de hersenen zorgt nicotine ervoor dat er dopamine vrij komt in het mesolimbische 'beloningssysteem'. Dat veroorzaakt een prettig gevoel, dat een roker graag wil herbeleven. Langdurige blootstelling aan nicotine veroorzaakt chronische veranderingen in het brein, waardoor rokers steeds sterker afhankelijk worden van nicotine. Zo ontstaat er een onbalans tussen een ineffectief cognitief controlesysteem (in de prefrontale cortex), en een overactief motivatiesysteem (oa. de amygdala en het ventrale striatum). Wanneer de belonende signalen vanuit het motivatiesysteem te sterk worden, kunnen ze de cognitieve controle overstemmen, waardoor rokers de drang om nog een sigaret op te steken niet kunnen weerstaan. Het motivatiesysteem wordt niet alleen geactiveerd door nicotine, maar ook door zogenaamde 'cues'; signalen uit de omgeving die geassocieerd worden met de belonende effecten van roken. Na verloop van tijd worden deze cues extreem opvallend en wekken ze het verlangen naar een (volgende) sigaret. Rokers richten en houden vaak automatisch hun aandacht op deze cues gevestigd. Dit proces wordt ook aandachtsbias (aandachtsverschuiving) genoemd en speelt een belangrijke rol in de instandhouding van rookverslaving.

Rookverslaving is dus voor een groot deel een consequentie van de effecten van nicotine in de hersenen. Een mogelijke strategie om te stoppen met roken is gericht op het tegengaan van deze effecten door er voor te zorgen dat nicotine de hersenen niet meer bereikt. Dit is het concept achter verschillende nicotinevaccins die op dit moment in ontwikkeling zijn. Het doel van ons eerste onderzoek (hoofdstuk 1) was om aan te tonen dat immunisatie stimulatie van de hersenen door nicotine vermindert. Daarnaast wilden we de effecten van nicotinevaccinatie op hersenactiviteit en gedrag aantonen tijdens het zien 
van cues gerelateerd aan roken en een werkgeheugentaak. Hiervoor werden achtenveertig rokende ( $\geq 10$ sigaretten/dag) mannen willekeurig ingedeeld in twee groepen. De ene groep werd behandeld met vijf $400 \mu \mathrm{g} / \mathrm{ml}$ injecties van het 3 'aminomethylnicotine P. aeruginosa r-Exoprotein conjugaat vaccin (NicVax) over een periode van 20 weken, de andere groep kreeg gedurende dezelfde periode vijf injecties met een niet-werkzame stof (placebo). Deelnemers werden onderzocht op twee momenten: een keer na toediening van nicotine en een keer na toediening van een niet-werkzame stof, en hen werd verzocht om in de 10 uur voorafgaand aan deze testmomenten niet te roken. Reactietijden en nauwkeurigheid werden gemeten tijdens een n-back werkgeheugentaak. Daarnaast werd doormiddel van een MRIscanner hersenactiviteit gemeten tijdens deze taak en tijdens het zien van rokengerelateerde cues. We vonden sterkere activatie tijdens het zien van rookcues dan tijdens het zien van neutrale cues in de bilaterale trans-occipitale sulcus, maar dit effect was niet bestand tegen een strenge statistische correctie. Verder was er geen verschil in hersenactiviteit tussen de Nicvax en placebo groepen tijdens het zien van rookcues en er is ook geen effect gevonden van de acute toediening van nicotine. Tijdens de n-back werkgeheugen taak vonden we dat hersenactiviteit in verschillende frontale en parietale hersengebieden toenam als de taak moeilijker werd. Maar ook tijdens deze taak waren er geen effecten van immunisatie of nicotinetoediening te zien. We kunnen dus concluderen dat behandeling met een nicotinevaccin de hoeveelheid nicotine in de hersenen niet zodanig heeft verminderd dat het invloed had op neurale en gedragsmatige reacties tijdens het zien van rookcues en een werkgeheugentaak. Daarom is dit vaccin waarschijnlijk geen effectief hulpmiddel om te stoppen met roken. NicVax zorgt mogelijk voor de productie van te weinig antilichamen, of antilichamen van te slechte kwaliteit (of een combinatie), waardoor het niet effectief is bij het stoppen met roken. Andere vaccins die leiden tot verbeterde antilichaamproductie kunnen in de toekomst effectiever blijken. Tot die tijd kan immuuntherapie nog niet worden uitgesloten als mogelijk hulpmiddel bij het stoppen en gestopt blijven met roken.

Naast het directe belonende effect van nicotine, zijn er ook andere processen die bijdragen aan de instandhouding van rookverslaving. Geconditioneerde reacties op rookcues in de omgeving spelen bijvoorbeeld een belangrijke rol. Om een stoppen-metroken behandeling te kunnen ontwikkelen gericht op deze reacties is het belangrijk om te weten welke mechanismes er aan ten grondslag liggen. In het onderzoek dat is beschreven in hoofdstuk 2 gebruikten we functionele MRI en een analyse die gericht is op patronen van hersenactiviteit om te onderzoeken of rookverslaving de vroege visuele verwerking kan beïnvloeden ten behoeve van roken-gerelateerde afbeeldingen. We verwachtten dat de neurale representatie van roken-gerelateerde afbeeldingen in de laterale occipitale cortex 
(LOC) verhoogd zou zijn na een periode van nicotine deprivatie en dat dit niet het geval zou zijn voor afbeeldingen die niet met roken te maken hebben. Veertien rokers $(\geq 10$ sigaretten per dag) ondergingen MRI scans op twee momenten: een keer na 10 uur niet gerookt te hebben en de andere keer mochten ze roken zo veel ze wilden. Met de MRI scanner werden regionale verschillen in het zuurstofgehalte in het bloed gemeten terwijl de proefpersonen keken naar reeksen afbeeldingen van sigaretten, potloden of stoelen. De functionele data werden geanalyseerd door middel van een patroonclassificatie aanpak. We vonden dat wanneer rokers gedepriveerd waren, het classificatiealgoritme in staat was om onderscheid te maken tussen patronen van activiteit gerelateerd aan visueel gelijkende objecten die wel (sigaretten) of niet aan roken gerelateerd waren (pennen), in linker en rechter LOC clusters. Dit onderscheid was niet te maken wanneer rokers recent gerookt hadden. Andere stimuluscombinaties (sigaretten en stoelen, pennen en stoelen) konden even goed van elkaar onderscheiden worden op basis van activiteitspatronen in LOC, ongeacht deprivatiestatus. In conclusie kunnen we dus zeggen dat het onderscheid tussen visueel vergelijkbare roken-gerelateerde en niet-roken-gerelateerde objecten in LOC toeneemt na een periode van nicotinedeprivatie. Dit laat zien dat de aandachtsbias in rokers mogelijk visuele objectverwerking beïnvloedt.

In een vervolgstudie (hoofdstuk 3) gebruikten we een multivariate 'zoeklicht'techniek om andere hersengebieden te identificeren die ook betrokken zijn bij de verwerking van rookcues onder invloed van nicotinedeprivatie. We gebruikten hiervoor de fMRI data van dezelfde veertien proefpersonen als in de vorige studie. Het zoeklicht identificeerde gebieden in bilaterale laterale occipitale cortex waarin bij nicotinegedepriveerde proefpersonen neurale activatiepatronen in reactie op rookcues beter te onderscheiden waren van die in reactie op neutrale cues. Daarnaast vonden we dat in de precentrale gyrus (hersenwinding) activatiepatronen in reactie op rookcues en neutrale cues juist beter te onderscheiden waren wanneer proefpersonen net gerookt hadden. Deze bevindingen ondersteunen de resultaten van onze vorige studie en tonen aan dat het mechanisme dat verantwoordelijk is voor de aandachtsbias van rokers voor rookcues waarschijnlijk neurale verwerking in het vroeg visuele systeem beïnvloedt.

Doordat het motivatiesysteem automatisch actief wordt bij het zien van rookcues ontstaat er een verlangen om te roken (craving) en krijgen deze cues steeds meer prioriteit van het aandachtssysteem. Deze processen spelen een belangrijke rol bij de instandhouding van rookverslaving en het tegengaan van deze processen zou een terugval in gestopte rokers mogelijk kunnen voorkomen. Wij hebben een online aandachtsbias modificatie (ABM) training ontwikkeld voor rokers met als doel hun automatische aandachtsverschuiving naar rookcues te verminderen (hoofdstuk 5). Rokers tussen de 18 en 60 jaar werden willekeurig ingedeeld in een ABM (208 deelnemers) en een controle conditie (190 deelnemers). Beide 
trainingen (ABM en controle) waren gebaseerd op een 'dot-probe' taak, waarbij proefpersonen werd gevraagd om zo snel mogelijk op een knop te drukken wanneer een stip op het computerscherm verscheen. Als rokers sneller reageerden op een stip wanneer die verscheen na een afbeelding waarop gerookt werd dan wanneer die verscheen na een afbeelding waar niet op gerookt werd, spraken we van aandachtsbias. In de ABM training verscheen de stip in $80 \%$ van de gevallen na een neutrale afbeelding, om de aandacht van de deelnemers impliciet naar deze niet-roken afbeeldingen te leiden. In de controle conditie verscheen de stip even vaak na een neutrale als een rookcue. Deelname bestond uit vijf training sessies op (semi-) aansluitende dagen. Aandachtsbias werd gemeten één dag voor de eerste en na de laatste training, en tijdens een follow-up sessie een week na de laatste meting. In de laatste meting hebben we een nieuwe set afbeeldingen toegevoegd om vast te stellen of het effect van de training te generaliseren was. Na vijf online trainingssessies was de aandachtsbias verminderd in de deelnemers in de ABM conditie, maar niet voor diegenen in de controle conditie. Dit effect was nog steeds aanwezig tijdens de follow-up een week later en bleek ook te generaliseren naar nieuwe, roken-gerelateerde afbeeldingen. Aandachtsbias modificatie is dus effectief in het verminderen van automatische aandachtsverschuiving naar rookcues en zou kunnen helpen om gestopte rokers minder gevoelig te maken voor een terugval.

Verschillende studies (waaronder die van ons) hebben recentelijk veelbelovende resultaten van $\mathrm{ABM}$ getoond op het verminderen van aandachtbias bij rokers. De effecten van ABM op rook verlangen en - gedrag zijn minder duidelijk. Daarom onderzochten wij of de effectiviteit van onze training beïnvloed werd door specifieke eigenschappen van rokers, zoals geslacht, opleiding, en mate van verslaving (hoofdstuk 6). Op de dataset van de vorige studie voerden we twee aparte factoriële univariate variantie analyses (ANOVA) uit om te bepalen wat de effecten van verschillende voorspellers waren op aandachtsbias tijdens baseline en na de training. Geen van de eigenschappen was van invloed op aandachtsbias voor de training. Het type training voorspelde de mate van aandachtsbias na de training, waarbij rokers na de ABM training minder bias hadden dan rokers die geen ABM training hadden gehad. Deze resultaten laten zien dat ABM rokers met verschillende demografische en roken-gerelateerde eigenschappen kan helpen bij het verlagen van hun aandachtsbias. Dat maakt ABM een zinvolle interventie die goed toepasbaar is in de praktijk.

Op basis van onze onderzoeken denken wij dat zowel anti-nicotine vaccins en $\mathrm{ABM}$ trainingen rokers kunnen helpen om (langdurig) gestopt te blijven. Rookverslaving bestaat echter uit complexe interacties tussen verschillende neurale en cognitieve processen. Daarom is het waarschijnlijk dat een behandeling alleen kan helpen bij het stoppen met roken en gestopt blijven wanneer die gericht is op een combinatie van deze aspecten. Wij 
geloven dat gecombineerd gebruik van verschillende interventietypes een einde kan maken aan de wereldwijde rookepidemie zodat we eindelijk vooruitgang boeken ten opzichte van de tijd van de cowboys. 


\section{SUPPLEMENTARY DATA}


Supplementary tables Chapter 4

Table S1a. Possible trial types in the dotprobe task

$\begin{array}{llll}\text { Type } & \begin{array}{l}\text { Location } \\ \text { smoking } \\ \text { cue }\end{array} & \begin{array}{l}\text { Duration } \\ \text { images }\end{array} & \text { Fixation } \\ & & & \end{array}$

\begin{tabular}{llll}
\hline \hline L44 & Left & $400 \mathrm{~ms}$ & $400 \mathrm{~ms}$ \\
L48 & Left & $400 \mathrm{~ms}$ & $800 \mathrm{~ms}$ \\
L64 & Left & $600 \mathrm{~ms}$ & $400 \mathrm{~ms}$ \\
L68 & Left & $600 \mathrm{~ms}$ & $800 \mathrm{~ms}$ \\
R44 & Right & $400 \mathrm{~ms}$ & $400 \mathrm{~ms}$ \\
R48 & Right & $400 \mathrm{~ms}$ & $800 \mathrm{~ms}$ \\
R64 & Right & $600 \mathrm{~ms}$ & $400 \mathrm{~ms}$ \\
R68 & Right & $600 \mathrm{~ms}$ & $800 \mathrm{~ms}$
\end{tabular}

Table S1b. Trial types in the assessment and in the ABM training \& control training

\begin{tabular}{|c|c|c|c|c|c|c|c|c|}
\hline $\begin{array}{l}\text { Assessment } \\
\text { Type }\end{array}$ & $\mathbf{N}$ & $\begin{array}{l}\text { N Con- } \\
\text { gruent* }\end{array}$ & $\begin{array}{l}A B M \\
\text { Type }\end{array}$ & $\mathbf{N}$ & $\begin{array}{l}\text { N Con- } \\
\text { gruent* }\end{array}$ & $\begin{array}{l}\text { Control } \\
\text { Type }\end{array}$ & $\mathbf{N}$ & $\begin{array}{l}\text { N Con- } \\
\text { gruent* }\end{array}$ \\
\hline L44 & 18 & 9 & L44 & 39 & 31 & L44 & 39 & 19 \\
\hline L48 & 18 & 9 & L48 & 39 & 32 & L48 & 39 & 20 \\
\hline L64 & 18 & 9 & L64 & 39 & 31 & L64 & 39 & 19 \\
\hline L68 & 18 & 9 & L68 & 39 & 31 & L68 & 39 & 20 \\
\hline R44 & 18 & 9 & R44 & 39 & 31 & R44 & 39 & 20 \\
\hline R48 & 18 & 9 & R48 & 39 & 32 & R48 & 39 & 19 \\
\hline R64 & 18 & 9 & R64 & 39 & 31 & R64 & 39 & 20 \\
\hline R68 & 18 & 9 & R68 & 39 & 31 & R68 & 39 & 19 \\
\hline Total & 144 & 72 & Total & 312 & 250 & Total & 312 & 156 \\
\hline
\end{tabular}


VALORISATION ADDENDUM

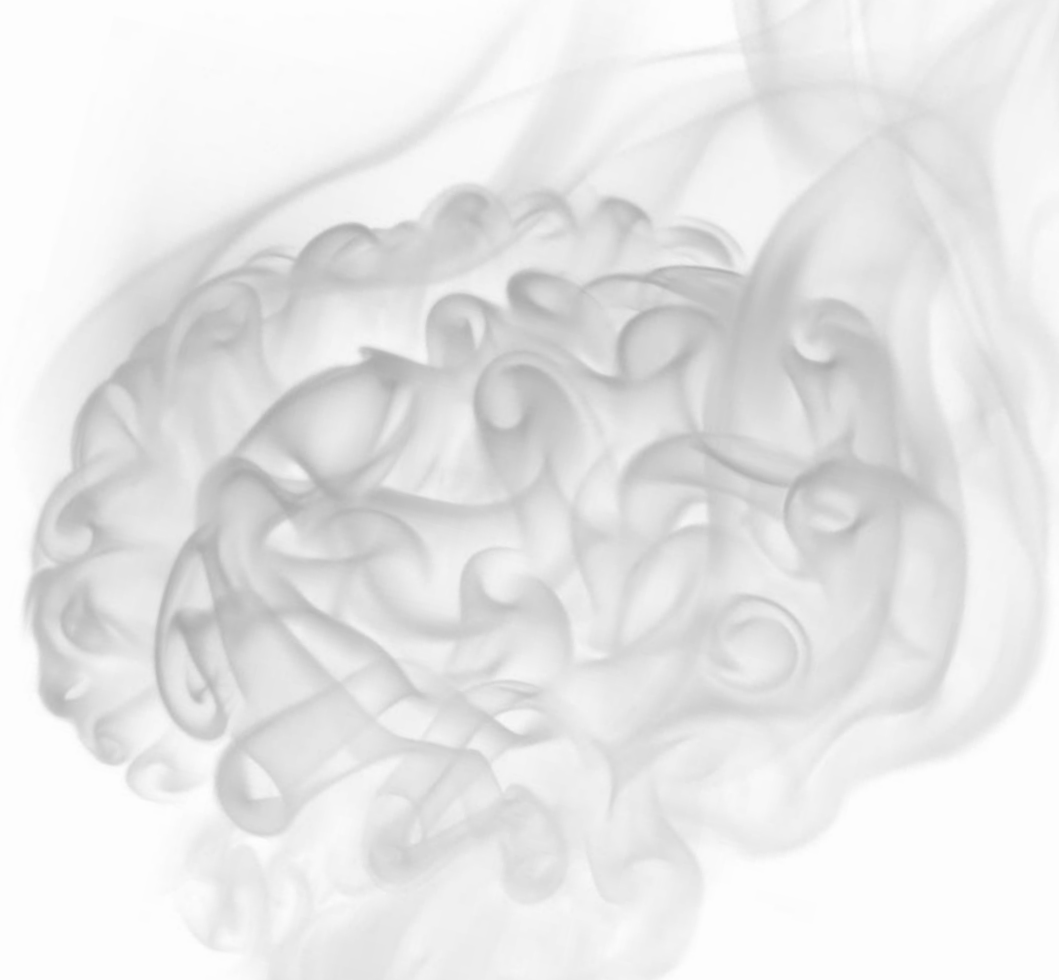


This addendum addresses the significance and the impact of the studies described in this dissertation. This is done by outlining in which way our studies are relevant to society, which target groups can benefit from our results, and which activities and products can be derived from our work. In addition, the innovative aspects of our research will be discussed, as well as how knowledge and ideas resulting from our studies have been and can be implemented.

\section{Societal relevance}

It is well known that smoking is one of the largest public health threats of current time; in fact, it is the number one preventable cause of death. Worldwide over one billion people smoke tobacco (World Health Organization, 2015), while in the Netherlands this number is close to 3.6 million (Nationaal Expertisecentrum Tabaksontmoediging, 2015). Smoking has serious adverse health effects and can cause life threatening illnesses such as coronary heart disease, chronic obstructive pulmonary disease (COPD), and various forms of cancer. The World Health Organization (WHO) estimates that approximately 6 million people die each year from tobacco-related disease as a result of direct tobacco use or second hand exposure to tobacco smoke (World Health Organization, 2015). In the Netherlands an estimated 20.000 people decease each year as a consequence of smoking (Nationaal Expertisecentrum Tabaksontmoediging, 2015).

Even though the detrimental consequences of smoking are clear, many smokers continue to smoke because they simply cannot manage to stay abstinent. In fact, a staggering $90 \%$ of smokers relapses within the first year after a quit attempt (Nationaal Expertisecentrum Tabaksontmoediging, 2015). Several treatments are available that have been proven effective in prolonging abstinence. However their additional benefit is strikingly low, as one year successful abstinence rates remain well below 50\% (Fiore et al., 2008; Rigotti, 2013). Viewing these numbers, it is clear that there is an urgent need for better treatments. In order to treat smoking addiction properly, it is crucial that this phenomenon and its underlying mechanisms are fully and correctly understood. The studies described in this thesis provide new insights in the neural (chapter 2 and 3) and cognitive mechanisms (discussion) underlying attention bias, and its malleability (chapter 4 and 5). The results of these studies provide new insights that can be helpful in the treatment of smoking addiction. We argue that smoking addiction is a complex process sustained by various neural as well as cognitive factors. We also state that targeting one single factor of smoking addiction, for instance eliminating the effects of nicotine by means of 
immunotherapy, is not sufficient to help people maintain abstinence as other factors will still assert their influence and trigger addictive behavior. Therefore we suggest that treatments for smoking addiction should target a combination of these various aspects in order to make it easier for ex-smokers to maintain abstinence.

\section{Target groups}

The results of the studies discussed in this dissertation are relevant to three distinct target groups. First of all, researchers in the field of smoking addiction or addiction in general can benefit from the new information we have added to the existing knowledge in this field. For example, we have shed a new light on the underlying neural mechanism of attention bias in smokers. Although our findings do not lead to a full and complete understanding of all factors maintaining smoking addiction, we have made a contribution to disentangling this complicated pathology. Researchers could build on these findings as well as the paradigms we have used, when designing new studies in the field. Secondly, (general) physicians and other health care professionals who want to help smokers reach and maintain abstinence could make use of our results when designing a treatment plan. And finally, our findings can be interesting to the general population of smokers who would like to learn more about their addiction and possible ways to quit without relapsing. Particularly, more awareness of the attentional processes underlying cigarette cravings could help smokers gain more control over their smoking habits.

\section{Activities and products}

Usually, the process of translating scientific findings in tangible activities, products or services is very slow, with many studies making small contributions to a whole. Similarly, our studies contribute to a better understanding of the processes involved in smoking addiction. Our increased knowledge about these processes can eventually lead to better interventions. We have also investigated two specific interventions, which were mainly aimed at relapse prevention. In the study described in our first chapter we examined the effects of a vaccine which was designed to prevent nicotine from entering the brain. Based on our findings and those from other studies (Esterlis et al., 2013; Hoogsteder, Kotz, van Spiegel, Viechtbauer, \& van Schayck, 2014) we suggest that this particular vaccine is not likely to be an effective aid in smoking cessation due to a suboptimal antibody response. 
Although this particular vaccine was not sufficiently successful, these findings may contribute to the development of other, more potent vaccines in the near future.

For another study we have developed an online training which was aimed at reducing attention bias in smokers (chapter 4). Attention bias causes smokers to focus their attention on craving triggering cues in their environment and has been associated with relapses after a quit attempt (Janes et al., 2010; Powell, Dawkins, West, Powell, \& Pickering, 2010; Spiegelhalder et al., 2011; Waters et al., 2003). Decreasing attention bias therefore may contribute to reduced craving and possibly prolonged abstinence. Our study has shown that five sessions of online attention bias modification (ABM) training successfully reduced attention bias in the general population of smokers. Follow-up studies are needed to find out whether the effects of this training are also clinically relevant. It would be very interesting to find out whether decreasing attention bias by means of ABM would also diminish cue-elicited craving and thereby can help prolong abstinence. If this is the case, this type of training is very well suitable to be applied through a mobile application.

\section{Innovation}

The research described in this dissertation is innovative in several ways. First, we were the first researchers to test the mechanism of action of nicotine immunization by means of functional magnetic resonance imaging (fMRI). Before the start of our study, other studies had only investigated immunogenicity, safety, and clinical effectiveness of nicotine vaccines (Cerny \& Cerny, 2009; Cornuz et al., 2008; Hartmann-Boyce, Cahill, Hatsukami, \& Cornuz, 2012; D.K. Hatsukami et al., 2011; Dorothy K. Hatsukami et al., 2005; Hoogsteder et al., 2014; Raupach, Hoogsteder, \& van Schayck, 2012). Nevertheless, the hypothesized mechanism of this treatment is that it precludes nicotine from entering and stimulating the brain. Therefore it is critical to assess changes nicotinergic stimulation of the brain after treatment in order to understand if and how immunization is effective.

Secondly, the studies in chapter 2 and 3 go beyond univariate fMRI analyses which are traditionally applied in our field. Instead, we made use of more advanced analysis methods such as multi voxel pattern analysis (MVPA) and a multivariate searchlight paradigm. MVPA analysis techniques employ the spatial distribution of neural responses, and thereby are able to pick up information that is too subtle to be discovered by traditional univariate analyses (De Martino et al., 2008). This enabled us to identify subtle differences 
in visual processing of smoking related and neutral cues, that could only be detected when smokers were deprived of nicotine but not when they were satiated.

Third, our ABM training was one of the first comprising multiple sessions and is still the only one that is fully web based. Before the start of our study, three studies had applied single session ABM for smokers, with varying results (Attwood, O'Sullivan, Leonards, Mackintosh, \& Munafò, 2008; Field, Duka, Tyler, \& Schoenmakers, 2009; McHugh, Murray, Hearon, Calkins, \& Otto, 2010). We hypothesized that multiple sessions of ABM training would be more successful in robustly reducing attention bias. During the time our study was executed three other studies were published in which multiple sessions of ABM were administered to smokers (Begh et al., 2015; Kerst \& Waters, 2014; Lopes, Pires, \& Bizarro, 2014). Two of these studies confirmed our hypothesis (Kerst \& Waters, 2014; Lopes et al., 2014), as did our own findings. Providing this type of training online has many advantages, for instance it may offer increased convenience, confidentiality, and reduced stigma for smokers (Brown et al., 2014). Moreover, it allowed us to reach a large sample of smokers that well reflects the composition of the general population of smokers.

And finally, we were the first to determine the influence of personal and smoking related characteristics of smokers on the effectiveness of ABM training. In order to bring $\mathrm{ABM}$ on the market as an aid for relapse prevention, it is important to understand for whom it will work and for whom it may not. Since attention bias seems to be higher in certain smokers (e.g. males (Littel, Euser, Munafò, \& Franken, 2012), heavy smokers (Mogg \& Bradley, 2002; Vollstädt-Klein et al., 2011; Zack, Belsito, Scher, Eissenberg, \& Corrigall, 2001), light smokers (Hogarth et al., 2003; Mogg et al., 2005) than others, it is possible that $\mathrm{ABM}$ is also more effective for certain subgroups of smokers. However our study demonstrated for the first time that $\mathrm{ABM}$ was equally successful for all smokers irrespective of age, gender, education, nicotine dependence, or their motivation to quit.

\section{Implementation}

We have undertaken and will undertake various efforts to ensure that the knowledge obtained from our studies reaches the target groups. First of all, I have presented most of our findings at several international conferences which are frequented by researchers and clinicians from all over the world. In addition, we aim to publish the research articles that constitute this thesis in various international scientific journals that target both scientific and clinical audiences. Moreover, in order to disseminate our findings to the general public we will also publish an article on a popular scientific website hosted by researchers from 
Maastricht University (www.brainmatters.nl). In addition, the findings will be brought to the attention of regional and national media by means of a press release from Maastricht University. Besides knowledge dissemination we are also planning to make our ABM training available to the public through a mobile application. Though, these plans are still in the early stages. 


\section{References}

Attwood, A. S., O’Sullivan, H., Leonards, U., Mackintosh, B., \& Munafò, M. R. (2008). Attentional bias training and cue reactivity in cigarette smokers. Addiction, 103(11), 1875-82.

Begh, R., Munafò, M. R., Shiffman, S., Ferguson, S. G., Nichols, L., Mohammed, M. A., ... Aveyard, P. (2015). Lack of attentional retraining effects in cigarette smokers attempting cessation: A proof of concept double-blind randomised controlled trial. Drug and Alcohol Dependence, 149, 158-165.

Brown, J., Michie, S., Geraghty, A. W. A., Yardley, L., Gardner, B., Shahab, L., ... West, R. (2014). Internet-based intervention for smoking cessation (StopAdvisor) in people with low and high socioeconomic status: a randomised controlled trial. Lancet. Respiratory Medicine, 2(12), 997-1006.

Cerny, E. H., \& Cerny, T. (2009). Vaccines against nicotine. Human Vaccines, 5(4), 200-5.

Cornuz, J., Zwahlen, S., Jungi, W. F., Osterwalder, J., Klingler, K., van Melle, G., ... Cerny, T. (2008). A vaccine against nicotine for smoking cessation: a randomized controlled trial. PloS One, 3(6), e2547.

De Martino, F., Valente, G., Staeren, N., Ashburner, J., Goebel, R., \& Formisano, E. (2008). Combining multivariate voxel selection and support vector machines for mapping and classification of fMRI spatial patterns. NeuroImage, 43(1), 44-58.

Esterlis, I., Hannestad, J. O., Perkins, E., Bois, F., D’Souza, D. C., Tyndale, R. F., ... O’Malley, S. S. (2013). Effect of a nicotine vaccine on nicotine binding to $\beta 2 *_{\text {- }}$ nicotinic acetylcholine receptors in vivo in human tobacco smokers. The American Journal of Psychiatry, 170(4), 399-407.

Field, M., Duka, T., Tyler, E., \& Schoenmakers, T. M. (2009). Attentional bias modification in tobacco smokers. Nicotine \& Tobacco Research, 11(7), 812-22.

Fiore, M. C., Jaén, C. R., Baker, T. B., Bailey, W. C., Benowitz, N. L., Curry, S. J., ... Wewers, M. E. (2008). Treating tobacco use and dependence: 2008 update. Rockville(MD): US Department of Health and Human Services 2008.

Hartmann-Boyce, J., Cahill, K., Hatsukami, D., \& Cornuz, J. (2012). Nicotine vaccines for smoking cessation. The Cochrane Database of Systematic Reviews, 8, CD007072.

Hatsukami, D. K., Jorenby, D. E., Gonzales, D., Rigotti, N. A., Glover, E. D., Oncken, C. 
A., ... Rennard, S. I. (2011). Immunogenicity and smoking-cessation outcomes for a novel nicotine immunotherapeutic. Clinical Pharmacology and Therapeutics, 89(3), $392-9$.

Hatsukami, D. K., Rennard, S., Jorenby, D., Fiore, M., Koopmeiners, J., de Vos, A., ... Pentel, P. R. (2005). Safety and immunogenicity of a nicotine conjugate vaccine in current smokers. Clinical Pharmacology and Therapeutics, 78(5), 456-67.

Hoogsteder, P. H. J., Kotz, D., van Spiegel, P. I., Viechtbauer, W., \& van Schayck, O. C. P. (2014). Efficacy of the nicotine vaccine 3'-AmNic-rEPA (NicVAX) co-administered with varenicline and counselling for smoking cessation: a randomized placebocontrolled trial. Addiction, 109(8), 1252-9.

Janes, A. C., Pizzagalli, D. A., Richardt, S., Frederick, B. deB., Chuzi, S., Pachas, G., ... Kaufman, M. J. (2010). Brain reactivity to smoking cues prior to smoking cessation predicts ability to maintain tobacco abstinence. Biological Psychiatry, 67(8), 722729.

Kerst, W. F., \& Waters, A. J. (2014). Attentional retraining administered in the field reduces smokers' attentional bias and craving. Health Psychology, 33(10), 1232-40.

Littel, M., Euser, A. S., Munafò, M. R., \& Franken, I. H. A. (2012). Electrophysiological indices of biased cognitive processing of substance-related cues: A meta-analysis. Neuroscience and Biobehavioral Reviews, 36(8), 1803-1816.

Lopes, F. M., Pires, A. V., \& Bizarro, L. (2014). Attentional bias modification in smokers trying to quit: a longitudinal study about the effects of number of sessions. Journal of Substance Abuse Treatment, 47(1), 50-7.

McHugh, R. K., Murray, H. W., Hearon, B. A., Calkins, A. W., \& Otto, M. W. (2010). Attentional bias and craving in smokers: the impact of a single attentional training session. Nicotine \& Tobacco Research, 12(12), 1261-4.

Mogg, K., \& Bradley, B. P. (2002). Selective processing of smoking-related cues in smokers: manipulation of deprivation level and comparison of three measures of processing bias. Journal of Psychopharmacology, 16(4), 385-392.

Nationaal Expertisecentrum Tabaksontmoediging (Institution). (2015). Roken: een aantal feiten op een rij. Utrecht.

Powell, J., Dawkins, L., West, R., Powell, J., \& Pickering, A. (2010). Relapse to smoking during unaided cessation: clinical, cognitive and motivational predictors. 
Psychopharmacology, 212(4), 537-49.

Raupach, T., Hoogsteder, P. H. J., \& van Schayck, O. C. P. (2012). Nicotine vaccines to assist with smoking cessation: current status of research. Drugs, 72(4), e1-16.

Rigotti, N. A. (2013). Smoking cessation in patients with respiratory disease: existing treatments and future directions. Lancet. Respiratory Medicine, 1(3), 241-50.

Spiegelhalder, K., Jähne, A., Kyle, S. D., Beil, M., Doll, C., Feige, B., \& Riemann, D. (2011). Is Smoking-related Attentional Bias a Useful Marker for Treatment Effects? Behavioral Medicine, 37(1), 26-34.

Vollstädt-Klein, S., Loeber, S., Winter, S., Leménager, T., von der Goltz, C., Dinter, C., ... Kiefer, F. (2011). Attention shift towards smoking cues relates to severity of dependence, smoking behavior and breath carbon monoxide. European Addiction Research, 17(4), 217-24.

Waters, A. J., Shiffman, S., Sayette, M. A., Paty, J. A., Gwaltney, C. J., \& Balabanis, M. H. (2003). Attentional bias predicts outcome in smoking cessation. Health Psychology, 22(4), 378-87.

World Health Organization. (2015). WHO | Tobacco Factsheet. Geneva: World Health Organization.

Zack, M., Belsito, L., Scher, R., Eissenberg, T., \& Corrigall, W. A. (2001). Effects of abstinence and smoking on information processing in adolescent smokers. Psychopharmacology, 153, 249-257. 
Neural and cognitive determinants of smoking addiction and cessation 


\section{DANKWOORD}

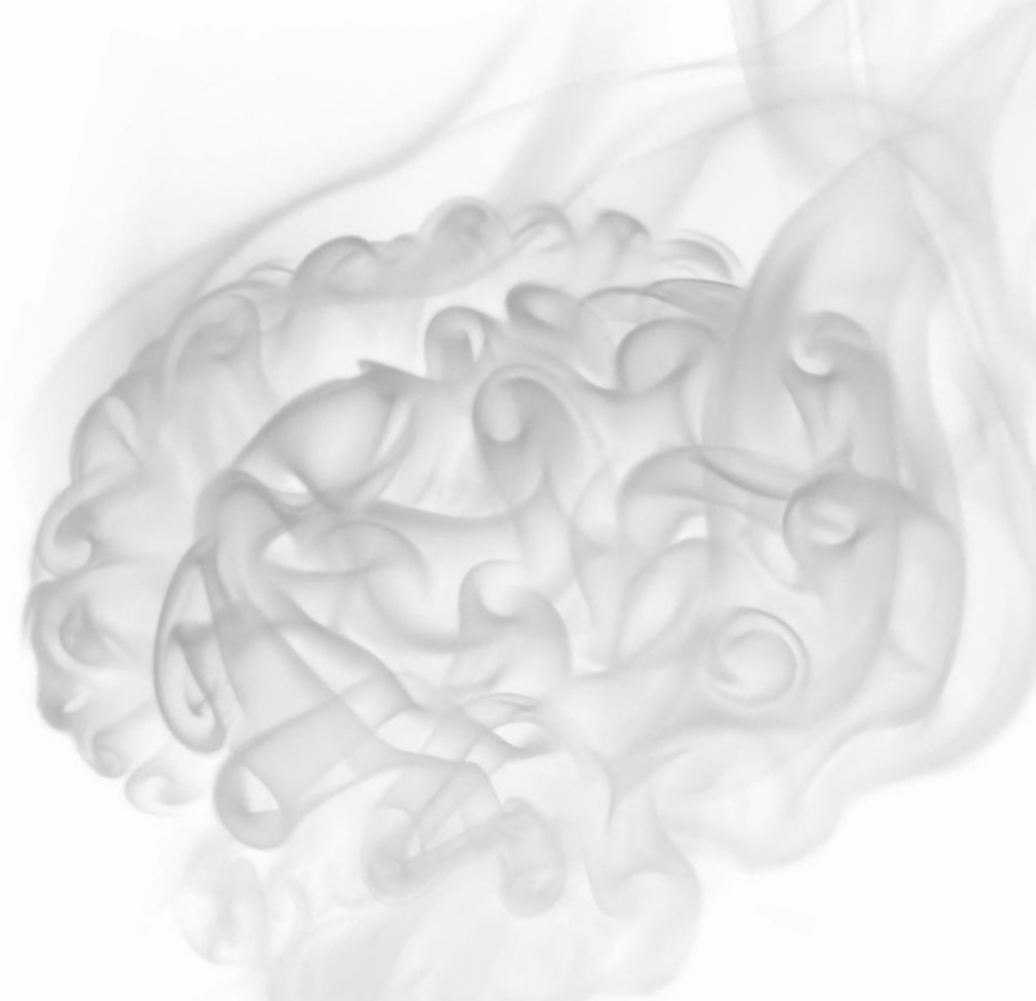


Het is zo ver, mijn proefschrift is af en straalt me tegemoet vanaf mijn computerscherm; een overzicht van alles wat ik in de afgelopen vier jaar heb meegemaakt en geleerd. En dat is nogal wat! Er zijn een hoop mensen die me in deze bijzondere tijd hebben geholpen, gesteund, opgevrolijkt, of hebben meegeleefd en die wil ik allemaal heel erg bedanken!

Allereerst veel dank aan mijn bijzondere promotieteam; prof. dr. Wim Riedel, prof. dr. Onno van Schayck en dr. Eric Vuurman. Met ieder een heel andere achtergrond brachten jullie mij nieuwe inzichten in mijn studies vanuit verschillende maar vaak ook complementaire invalshoeken. Ondanks dat er meestal al drie meningen in het spel waren stonden jullie altijd open voor een vierde. Bedankt dat jullie zo open stonden voor mijn ideeën en me de vrijheid gaven om zelf beslissingen te nemen. Eric, als dagelijkse begeleider was jij wat dichter betrokken bij het uitvoeren van het onderzoek en dat heb je geweten; van scannen in het weekend, tot een scanner die het liet afweten, en een verdwenen stagiair. Wat er ook gebeurde, jij stond onvoorwaardelijk achter mij en kwam voor me op waar nodig. Dat gaf mij het vertrouwen om keuzes te maken en heeft me doen groeien als onderzoeker en als mens. Bedankt daarvoor.

Members of the assessment committee; prof. dr. Jan Ramaekers, prof. dr. Daniel Kotz, prof. dr. Marcus Munafo, dr. Sandra Mulkens, thank you very much for reading and assessing this thesis. Bedankt prof dr. Jean Muris, prof. dr. Rob Ruijter, dr. Maartje Luijten en dr. Vincent van de Ven voor jullie deelname aan mijn promotiecommissie.

Job, als coauteur op het merendeel van mijn artikelen heb jij een belangrijk aandeel gehad in de totstandkoming van dit proefschrift. Ik heb de afgelopen vier jaren ongelofelijk veel van jou geleerd; van hoe belangrijk het is om je taken te synchroniseren met de scanner, tot geavanceerde machine learning data analysetechnieken, maar ook over hoe om te gaan met de tegenslagen en moeilijke momenten in een $\mathrm{PhD}$ traject. En ik kan met zekerheid zeggen dat dit boekje er in deze hoedanigheid zonder jou niet was geweest. Heel erg bedankt voor al je tijd, oplossingen, en bovenal je vriendschap.

Anique, jij hebt de fundering gelegd voor mijn eerste studie. Bedankt voor al je werk en je zeer gestructureerde administratie van alles, waar ik nog menigmaal op terug heb kunnen vallen.

Het onderzoeksteam van de NicVax studies; keuringsartsen Philippe Hoogsteder en Piet Portegijs, onderzoeksassistentes Pauline, Arja en Jeannique, en studenten Ilona en Roelof, bedankt voor de prettige samenwerking en goede zorg voor mijn proefpersonen. Daarnaast ook veel dank aan mijn eigen studentassistenten Christoph en Marcel, voor jullie aanwezigheid tijdens het scannen en hulp met het preprocessen van een hele hoop fMRI 
data. Ook wil ik Lotty bedanken voor alle technische en logistieke hulp bij het scannen. Anita, bedankt dat je altijd klaar stond om op te treden als backup bij de scanner en om bloed te prikken bij de proefpersonen. Tot slot Lizzy Vuurman, heel erg bedankt dat jij zelfs 's avonds laat of in het weekend bereid was om speciaal naar de universiteit te komen om bloed af te nemen bij mijn proefpersonen.

Uiteraard ook veel dank aan alle proefpersonen die zich hebben ingezet voor mijn studies; zonder jullie was dit proefschrift er nooit geweest!

Michiel Vestjens en Charlie Bonnemayer, dankjulliewel voor de technische ondersteuning van mijn laatste studie. Ik kon altijd bij jullie aankloppen met een vraag, en als ik dat deed gingen jullie er meteen mee aan de slag; wat een fijne samenwerking!

A special thanks to my darling roomies Luciana and Janelle. Thank you both so much for welcoming me in the department as well as in your personal and social lives. You have thought me all important things a $\mathrm{PhD}$ student should know; from the practical aspects of conducting research to how many eggs to eat a week, and to make sure to go to the gym every now and then. In spite of our very different personalities (or perhaps because of them), I think the three of us were a perfect match. I have so many great memories of the fun times we shared, also with Aimee, Joy, and Eliza. Joy, what a pleasure it was to meet such a likeminded person. Eliza, your sparkling, slightly crazy and very kind personality can make anyone smile on a dark day. Thank you both for your friendship!

Natuurlijk wil ik ook mijn nieuwe lichting roomies, Eri, Christine en Eva, heel erg bedanken. Het is bijzonder hoe we elkaar zo snel, zo goed hebben leren kennen. Bedankt dat ik alles met jullie kon delen, voor het luisteren naar mijn geklaag, en voor jullie ontzettend lieve support toen de druk toenam en het einde (van mijn proefschrift, mijn baan, mijn leven! ;)) in zicht kwam. Eva, toen ik het dieptepunt bereikt had gaf je mij met een moederlijk lieve knuffel de moed om nog even door te zetten en mijn proefschrift opnieuw af te schrijven. Dankjulliewel.

I was also lucky to have three lovely girls next door; Eliza, Laura, and Franziska, thank you for the many lunches, coffee moments, teabags, chocolates, and other types of mental support! To celebrate finishing my thesis with you (including Christine and Stefan) and your surprise bottle of prosecco was the best feeling in the world!

Also many thanks to Joep, Robbie, Nick, Judith, Sanae, Anna, and Inge for many lovely lunches, coffee breaks and other fun activities that kept me going. I also want to thank all other colleagues of the department of Neuropsychology and Psychopharmacology for all 
informative and joyful moments we have shared in the past years. What a pleasure it was to be part of this great department! Annemie, een speciaal bedankje voor jou. Zo fijn dat je altijd klaar stond om mijn vragen te beantwoorden en administratieve regel-dingetjes uit handen te nemen.

Lieve Willie en Steef, ik ben zo blij dat jullie mijn paranimfen zijn! Stefan, wij zijn het PhD avontuur samen begonnen vier jaar geleden en het was heel fijn om iemand te hebben die tegelijk met mij die weg vol ups en downs bewandelde. De vele koffiemomentjes hebben onze vriendschap sterker gemaakt en daar ben ik heel dankbaar voor. Willemijn, wij bewandelen al wat langer dezelfde weg en ik was dan ook heel blij toen bleek dat we dit $\mathrm{PhD}$ avontuur ook weer samen zouden gaan beleven. Ik heb genoten van onze avondjes samen, thuis of op ons favoriete terras, met een wijntje of een biertje, waarop we ons met veel lachen konden ontladen van de dagelijks spanningen. Dat gebeurde trouwens ook tijdens onze fietsritjes naar huis en tijdens het fitnessen. Ik kan alles met je delen en je staat altijd klaar om mee te denken over de dingen waar ik me mee bezig houd. En als ik me te veel laat gaan, weet jij me weer met beide benen op de grond te zetten. Je was er voor me als ik het even moeilijk had, zelfs toen je ver weg was; jouw lieve kaartje vanuit Chicago kwam precies op het goede moment. Dankjewel voor alles lieve Willie!

Lieve Sannie, jou wil ik ook nog even speciaal bedanken voor je interesse, kritische vragen en support de afgelopen jaren. Wendy, bedankt dat je last-minute nog bereid was om mee te denken over de vormgeving van mijn kaft. Natuurlijk ook dank aan alle sardientjes vriendjes, inclusief Heleen, Yoanne, Peter, Loes, Tamara en Maarten voor de vele gezellige spelletjes-, stap-, drankjes-, meezing- en sup-del-spec- avondjes en weekendjes! Dat er nog maar veel mogen volgen!

Lieve Bredase maatjes, Anne, Marieke, Sanne, Daniëlle, Laura, Amanda en Marlou, wat ben ik trots dat onze vriendschap al zoveel jaren en kilometers afstand heeft overleefd! We hebben de afgelopen jaren heel wat mooie momenten gedeeld, maar hebben ook ondervonden dat we er in moeilijke tijden voor elkaar zijn. Bedankt voor jullie vriendschap, jullie zijn voor mij de parels van het zuiden!

Lieve pap en Anja, bedankt voor jullie onvoorwaardelijk support. Jullie hebben me geleerd om, ook als mijn werk veel aandacht opeiste, te blijven genieten van leuke dingen in het leven. Zoals bijvoorbeeld van de mooie reisjes met het gezin. Bedankt daarvoor!

Lieve Robs, Bobbie en Nien, ik ben zo trots om jullie zus te zijn. Blijf altijd jezelf, jullie zijn de leuksten! 
Lieve moetie, er is waarschijnlijk geen moeder op de wereld zo trots als jij! Dankjewel dat je er altijd voor me bent en naar me luistert. Helaas heb ik je met mijn onderzoek niet van je verslaving af kunnen brengen, maar ik ben heel blij dat die langzamerhand overgenomen wordt door een nieuwe, namelijk René!

It always seems impossible, until it's done.

- Nelson Mandela

I did it! 
Neural and cognitive determinants of smoking addiction and cessation 


\section{CURRICULUM VITAE}

Anne Havermans was born on May $17^{\text {th }}, 1988$ in Breda, The Netherlands. She graduated from secondary education (VWO) at Onze Lieve Vrouwe Lyceum in Breda in 2006, and continued her education studying psychology at the Faculty of Psychology and Neuroscience (FPN) at Maastricht University. After obtaining a Bachelor's degree in 2009, she started the two-year research master in Cognitive and Clinical Neuroscience (specialization Neuropsychology). During her research internship under supervision of Prof. Dr. Alexander Sack at the department of Cognitive Neuroscience, she studied the relation between the lateralization of attention and facial emotion recognition by means of transcranial magnetic stimulation (TMS). This internship triggered Anne's enthusiasm for research, and after obtaining her Research Master's degree in 2011 she straightaway started a combined PhD at the department of Neuropsychology and Psychopharmacology (FPN) and CAPHRI School for Public Health and Primary Care (Faculty of Health, Medicine, and Life Sciences). During her PhD, Anne also gained extensive teaching experience and in 2015 she received her Basic Teaching Qualification Certificate. Currently, she continues to work as a teacher at Maastricht University. 
